\title{
Enantioselective Diels-Alder Reactions of Cyclohexa-1,3-diene and Chalcones Catalyzed by Intramolecular Silicon-Sulfur Lewis Pairs as Chiral Lewis Acids
}

\author{
Polina Shaykhutdinova and Martin Oestreich \\ Institut für Chemie, Technische Universität Berlin, \\ Strasse des 17. Juni 115, 10623 Berlin, Germany \\ martin.oestreich@tu-berlin.de
}

\section{Supporting Information}

\section{Table of Contents}

1 General Information

2 Experimental Details for the Synthesis of the Hydrosilanes $\quad$ S5

2.1 General Procedure for the Preparation of 2,2'-Dihalo-1,1'-binaphthyls (GP1) S5

2.1.1 rac-2-Bromo-2'-phenylthio-1,1'-binaphthyl (rac-9) S5

2.1.2 (S)-2-lodo-2'-phenylthio-1,1'-binaphthyl $[(S)-7] \quad S 6$

2.1.3 (R)-2-lodo-2'-phenylthio-1,1'-binaphthyl $[(R)-7] \quad S 6$

2.2 General Procedure for the Synthesis of the Diastereomeric Hydrosilanes (GP2) $\quad$ S7

2.2.1 (4RS,11bS)-4-(2'-Phenylthio-[1,1'-binaphthalen]-2-yl)-4,5-dihydro-3H-dinaphtho $\left[2,1-c: 1^{\prime}, 2^{\prime}-e\right]$ silepine $[(S, R S)-11]$

2.2.2 (4S,11bS)-4-(2'-Phenylthio-[1,1'-binaphthalen]-2-yl)-4,5-dihydro-3H-dinaphtho $\left[2,1-c: 1^{\prime}, 2 '-e\right]$ silepine $[(S, S)-11]$

2.2.3 (4R,11bS)-4-(2'-Phenylthio-[1,1'-binaphthalen]-2-yl)-4,5-dihydro-3H-dinaphtho $\left[2,1-c: 1^{\prime}, 2^{\prime}-e\right]$ silepine $[(S, R)-11]$

3 General Procedure for the Generation of Intramolecularly Sulfur-Stabilized Silicon Cations (GP3)

3.1 (4RS,11bS)-4-(2'-Phenylthio-[1,1'-binaphthalen]-2-yl)-4,5-dihydro-3H-dinaphtho $\left[2,1-c: 1^{\prime}, 2^{\prime}-e\right]$ silepinylium Tetrakis(pentafluorophenyl)borate $[(S, R S)-5]$ 
[2,1-c:1',2'-e]silepinylium Tetrakis(pentafluorophenyl)borate [(S,S)-5]

3.3 (4R,11bS)-4-(2'-Phenylthio-[1,1'-binaphthalen]-2-yl)-4,5-dihydro-3H-dinaphtho

$\left[2,1-c: 1^{\prime}, 2 '-e\right]$ silepinylium Tetrakis(pentafluorophenyl)borate $[(S, R)-5]$

3.4 (4R,11bS)-4-(2'-Phenylthio-[1,1'-binaphthalen]-2-yl)-4,5-dihydro-3H-dinaphtho [2,1-c:1',2'-e]silepinylium Tetrakis(pentafluorophenyl)borate Benzophenone Adduct $\left[(\mathrm{S}, \mathrm{R})-\mathbf{5} \cdot \mathrm{O}=\mathrm{CPh}_{2}\right]$

4 General Procedure for the Preparation of Chalcones 12-20 by ClaisenSchmidt Condensation (GP4)

4.1 (E)-3-Phenyl-1-(p-tolyl)prop-2-en-1-one (12)

4.2 (E)-1-(4-(tert-Butyl)phenyl)-3-phenylprop-2-en-1-one (13)

4.3 (E)-1-([1,1'-biphenyl]-4-yl)-3-phenylprop-2-en-1-one (14)

4.4 (E)-3-Phenyl-1-(o-tolyl)prop-2-en-1-one (15)

4.5 (E)-1-(2-Methoxyphenyl)-3-phenylprop-2-en-1-one (16)

4.6 (E)-1-Mesityl-3-phenylprop-2-en-1-one (17)

4.7 (E)-1-(Naphthalen-1-yl)-3-phenylprop-2-en-1-one (18)

$4.8 \quad(E)-1-(N a p h t h a l e n-2-y l)-3-p h e n y l p r o p-2-e n-1-o n e(19)$

4.9 (E)-1,3-Di(naphthalen-2-yl)prop-2-en-1-one (20)

5 General Procedure for Diels-Alder Reactions Catalyzed by Sulfur-Stabilized Silicon Cations (GP5)

5.1 endo-1-(-3-Phenylbicyclo[2.2.2]oct-5-en-2-yl)ethan-1-one (3)

5.2 endo-3-Phenylbicyclo[2.2.2]oct-5-en-2-yl)(p-tolyl)methanone (21)

5.3 endo-(4-(tert-Butyl)phenyl)(-3-phenylbicyclo[2.2.2]oct-5-en-2-yl)methanone (22)

5.4 endo-[1,1'-Biphenyl]-4-yl(-3-phenylbicyclo[2.2.2]oct-5-en-2-yl)methanone (23)

5.5 endo-3-Phenylbicyclo[2.2.2]oct-5-en-2-yl)(o-tolyl)methanone (24)

5.6 endo-(2-Methoxyphenyl)(-3-phenylbicyclo[2.2.2]oct-5-en-2-yl)methanone (25)

5.7 endo-Naphthalen-1-yl(-3-phenylbicyclo[2.2.2]oct-5-en-2-yl)methanone (27) S27

5.8 endo-Naphthalen-2-yl(-3-phenylbicyclo[2.2.2]oct-5-en-2-yl)methanone (28)

5.9 endo-Naphthalen-2-yl(-3-(naphthalen-2-yl)bicyclo[2.2.2]oct-5-en-2-yl)methanone (29)

5.10 endo-Phenyl(-3-phenylbicyclo[2.2.2]oct-5-en-2-yl)methanone (31)

Figures S1-S2. ${ }^{1} \mathrm{H}$ and ${ }^{13} \mathrm{C}$ NMR Spectra of rac-9

Figures S3-S5. ${ }^{1} \mathrm{H},{ }^{13} \mathrm{C}$ and ${ }^{29} \mathrm{Si}$ DEPT NMR Spectra of $(S, R S)-11$ S33-S35

Figures S6-S8. ${ }^{1} \mathrm{H},{ }^{13} \mathrm{C}$ and ${ }^{29} \mathrm{Si}$ DEPT NMR Spectra of $(S, S)-11$ 
Figures S9-S11. ${ }^{1} \mathrm{H},{ }^{13} \mathrm{C}$ and ${ }^{29} \mathrm{Si}$ DEPT NMR Spectra of $(S, R)-11$

S39-S41

Figures S12-S16. ${ }^{1} \mathrm{H},{ }^{13} \mathrm{C},{ }^{11} \mathrm{~B},{ }^{19} \mathrm{~F}$ and ${ }^{1} \mathrm{H},{ }^{29} \mathrm{Si} \mathrm{HMQC} \mathrm{NMR} \mathrm{Spectra} \mathrm{of}(S, R S)-5$

S42-S46

Figures S17-S22. ${ }^{1} \mathrm{H},{ }^{13} \mathrm{C},{ }^{11} \mathrm{~B},{ }^{19} \mathrm{~F},{ }^{29} \mathrm{Si}$ DEPT and ${ }^{1} \mathrm{H},{ }^{29} \mathrm{Si} \mathrm{HMQC} \mathrm{NMR} \mathrm{Spectra}$ of $(S, S)-5$

S46-S51

Figures S23-S27. ${ }^{1} \mathrm{H},{ }^{13} \mathrm{C},{ }^{11} \mathrm{~B},{ }^{19} \mathrm{~F}$ and ${ }^{1} \mathrm{H},{ }^{29} \mathrm{Si} \mathrm{HMQC}$ NMR Spectra of $(S, R)-5$

S53-S57

Figures S28-S33. ${ }^{1} \mathrm{H},{ }^{13} \mathrm{C},{ }^{11} \mathrm{~B},{ }^{19} \mathrm{~F},{ }^{29} \mathrm{Si}$ DEPT and ${ }^{1} \mathrm{H},{ }^{29} \mathrm{Si} \mathrm{HMQC} \mathrm{NMR} \mathrm{Spectra}$ of $(S, R)-5 \cdot \mathrm{O}=\mathrm{CPh}_{2}$

S58-S63

Figures S34-S35. ${ }^{1} \mathrm{H}$ and ${ }^{13} \mathrm{C}$ NMR Spectra of 12

S64-S65

Figures S36-S37. ${ }^{1} \mathrm{H}$ and ${ }^{13} \mathrm{C}$ NMR Spectra of 13 S66-S67

Figures S38-S39. ${ }^{1} \mathrm{H}$ and ${ }^{13} \mathrm{C}$ NMR Spectra of 14

S68-S69

Figures S40-S41. ${ }^{1} \mathrm{H}$ and ${ }^{13} \mathrm{C}$ NMR Spectra of 15

S70-S71

Figures S42-S43. ${ }^{1} \mathrm{H}$ and ${ }^{13} \mathrm{C}$ NMR Spectra of 16

S72-S73

Figures S44-S45. ${ }^{1} \mathrm{H}$ and ${ }^{13} \mathrm{C}$ NMR Spectra of 17 S74-S75

Figures S46-S47. ${ }^{1} \mathrm{H}$ and ${ }^{13} \mathrm{C}$ NMR Spectra of 18 S76-S77

Figures S48-S49. ${ }^{1} \mathrm{H}$ and ${ }^{13} \mathrm{C}$ NMR Spectra of 19

S78-S79

Figures S50-S51. ${ }^{1} \mathrm{H}$ and ${ }^{13} \mathrm{C}$ NMR Spectra of 20

S80-S81

Figures S52-S53. ${ }^{1} \mathrm{H}$ and ${ }^{13} \mathrm{C}$ NMR Spectra of 3

S82-S83

Figures S54-S55. ${ }^{1} \mathrm{H}$ and ${ }^{13} \mathrm{C}$ NMR Spectra of 21 S84-S85

Figures S56-S57. ${ }^{1} \mathrm{H}$ and ${ }^{13} \mathrm{C}$ NMR Spectra of 22 S86-S87

Figures S58-S59. ${ }^{1} \mathrm{H}$ and ${ }^{13} \mathrm{C}$ NMR Spectra of 23 S88-S89

Figures S60-S61. ${ }^{1} \mathrm{H}$ and ${ }^{13} \mathrm{C}$ NMR Spectra of 24 S90-S91

Figures S62-S63. ${ }^{1} \mathrm{H}$ and ${ }^{13} \mathrm{C}$ NMR Spectra of 25 S92-S93

Figures S64-S65. ${ }^{1} \mathrm{H}$ and ${ }^{13} \mathrm{C}$ NMR Spectra of 27 S94-S95

Figures S66-S67. ${ }^{1} \mathrm{H}$ and ${ }^{13} \mathrm{C}$ NMR Spectra of 28 S96-S97

Figures S68-S69. ${ }^{1} \mathrm{H}$ and ${ }^{13} \mathrm{C}$ NMR Spectra of 29 S98-S99

Figures S70-S71. ${ }^{1} \mathrm{H}$ and ${ }^{13} \mathrm{C}$ NMR Spectra of 31 


\section{$1 \quad$ General Information}

All reactions were performed in flame-dried glassware using an MBraun glovebox $\left(\mathrm{O}_{2}<0.5 \mathrm{ppm}\right.$, $\mathrm{H}_{2} \mathrm{O}<0.5 \mathrm{ppm}$ ) or conventional Schlenk techniques under a static pressure of argon (glovebox) or nitrogen. Liquids and solutions were transferred with syringes. Solvents were dried and purified following standard procedures. Technical grade solvents for extraction or chromatography were distilled prior to use. $\mathrm{C}_{6} \mathrm{D}_{6}, \mathrm{CDCl}_{3}$, and $\mathrm{CD}_{2} \mathrm{Cl}_{2}$ were dried over $4 \AA$ molecular sieves, and 1,2$\mathrm{Cl}_{2} \mathrm{C}_{6} \mathrm{D}_{4}$ was dried over $\mathrm{CaH}_{2}$, distilled, and stored under argon. Cyclohexa-1,3-diene (1) was distilled from $\mathrm{CaH}_{2}$ prior to use. Dienophiles were dried by azeotropic distillation with benzene prior to use. Trityl tetrakis(pentafluorophenyl)borate $\left(\left[\mathrm{Ph}_{3} \mathrm{C}\right]^{+}\left[\mathrm{B}\left(\mathrm{C}_{6} \mathrm{~F}_{5}\right)_{4}\right]^{-}\right.$) was prepared following a reported procedure, ${ }^{[\mathrm{S} 1 \mathrm{a}, \mathrm{b}]}$ recrystallized from $\mathrm{CH}_{2} \mathrm{Cl}_{2} / n$-pentane, and stored in a glovebox. Analytical thin layer chromatography (TLC) was performed on silica gel 60 F254 glass plates by Merck. Flash column chromatography was performed on silica gel LC60A $(40-63 \mu \mathrm{m})$ by Grace using the indicated solvents. ${ }^{1} \mathrm{H},{ }^{13} \mathrm{C},{ }^{11} \mathrm{~B},{ }^{19} \mathrm{~F}$, and ${ }^{29} \mathrm{Si} N \mathrm{NR}$ spectra were recorded in $\mathrm{CDCl}_{3}, \mathrm{C}_{6} \mathrm{D}_{6}$, or $1,2-$ $\mathrm{Cl}_{2} \mathrm{C}_{6} \mathrm{D}_{4}$ on Bruker AV500 or Bruker AV400 instruments. Chemical shifts are reported in parts per million (ppm) and are referenced to the residual solvent resonance as the internal standard $\left(\mathrm{CHCl}_{3}\right.$ : $\delta=7.26$ ppm for ${ }^{1} \mathrm{H}$ NMR and $\mathrm{CDCl}_{3}: \delta=77.16 \mathrm{ppm}$ for ${ }^{13} \mathrm{C}$ NMR, $\mathrm{C}_{6} \mathrm{D}_{5} \mathrm{H}: \delta 7.16 \mathrm{ppm}$ for ${ }^{1} \mathrm{H}$ NMR and $\mathrm{C}_{6} \mathrm{D}_{6}: \delta 128.06 \mathrm{ppm}$ for ${ }^{13} \mathrm{C} \mathrm{NMR}, \mathrm{CDHCl}_{2}: \delta 5.32 \mathrm{ppm}$ for ${ }^{1} \mathrm{H} \mathrm{NMR}$ and $\mathrm{CD}_{2} \mathrm{Cl}_{2}: \delta 53.84 \mathrm{ppm}$ for ${ }^{13} \mathrm{C}$ NMR; $1,2-\mathrm{Cl}_{2} \mathrm{C}_{6} \mathrm{D}_{3} \mathrm{H}: \delta 6.94$ and $7.20 \mathrm{ppm}$ for ${ }^{1} \mathrm{H}$ NMR and $1,2-\mathrm{Cl}_{2} \mathrm{C}_{6} \mathrm{D}_{4}: \delta 127.1,130.1$, and $132.5 \mathrm{ppm}$ for ${ }^{13} \mathrm{C} \mathrm{NMR}$ ). Data are reported as follows: chemical shift, multiplicity (br $\mathrm{s}=$ broad singlet, $\mathrm{s}=$ singlet, $\mathrm{d}=$ doublet, $\mathrm{dd}=$ doublet of doublets, $\mathrm{ddd}=$ doublet of doublet of doublets, $\mathrm{t}=$ triplet, $\mathrm{q}=$ quartet, $\mathrm{m}=$ multiplet, $\mathrm{m}_{c}=$ centrosymmetric multiplet, $\mathrm{br} \mathrm{m}=$ broad multiplet), coupling constants $(\mathrm{Hz})$, and integration. ${ }^{1} \mathrm{H},{ }^{29} \mathrm{Si} \mathrm{HMQC}$ NMR spectra were measured with a coupling constant of $7.0 \mathrm{~Hz}$ for the ${ }^{3} \mathrm{~J}_{\mathrm{H}, \mathrm{Si}}$ coupling. The peak intensities in the ${ }^{1} \mathrm{H},{ }^{29} \mathrm{Si} \mathrm{HMQC}$ NMR spectra cannot be correlated to the amount of compound. Infrared (IR) spectra were recorded on an Agilent Technologies Cary 630 FTIR spectrophotometer equipped with an ATR unit and are reported as wavenumbers $\left[\mathrm{cm}^{-1}\right]$ (br = broad, $\mathrm{w}=$ weak, $\mathrm{m}=$ medium, $\mathrm{s}=$ strong, vs = very strong). Gas liquid chromatography (GLC) was performed on an Agilent Technologies 7820A gas chromatograph equipped with a HP-5 capillary column $(30 \mathrm{~m} \times 0.32 \mathrm{~mm}, 0.25 \mu \mathrm{m}$ film thickness $)$ using the following program: $\mathrm{N}_{2}$ carrier gas, column flow: $1.74 \mathrm{~mL} \mathrm{~min}^{-1}$, injection temperature: $250{ }^{\circ} \mathrm{C}$, detector temperature: $300{ }^{\circ} \mathrm{C}$; temperature program: start temperature $40{ }^{\circ} \mathrm{C}$, heating rate $10{ }^{\circ} \mathrm{C}$ $\mathrm{min}^{-1}$, end temperature $280{ }^{\circ} \mathrm{C}$ for $10 \mathrm{~min}$. Melting points (m.p.) were determined with a Stuart Scientific SMP20 melting point apparatus and are not corrected. Optical rotations were measured on a Schmidt \& Haensch Polatronic $\mathrm{H} 532$ polarimeter with $[\alpha]_{D}$ values reported in $\left[{ }^{0} 10^{-1} \mathrm{~cm}^{2} \mathrm{~g}^{-1}\right]$;

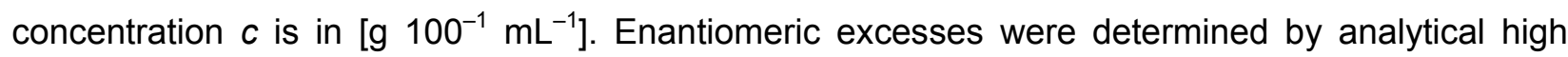


performance liquid chromatography (HPLC) analysis on an Agilent Technologies 1200 Infinity instrument with a Daicel Chiralcel OD-H column as the chiral stationary phase ( $n$-heptane/i-PrOH mixtures as solvents). High-resolution mass spectrometry (HRMS) and elemental analysis were performed at the Analytical Facility of the Institut für Chemie, Technische Universität Berlin.

\section{Experimental Details for the Synthesis of the Hydrosilanes}

\subsection{General Procedure for the Preparation of 2,2'-Dihalo-1,1'-binaphthyls (GP1) ${ }^{[\mathrm{S} 2]}$}

To a solution of 2,2'-dihalo-1,1'-binaphthyl (1.00 equiv) in THF $(0.18 \mathrm{M})$ is added a hexane solution of $n \mathrm{BuLi}$ ( 0.95 equiv) over a period of $70 \mathrm{~min}$ at $-78^{\circ} \mathrm{C}$, and the reaction mixture is then maintained at this temperature for $1 \mathrm{~h}$. A solution of diphenyl disulfide (1.2 equiv) in THF (0.44 M) is slowly added, the mixture is subsequently allowed to slowly warm to room temperature, followed by stirring overnight. The reaction is quenched by the addition of saturated aqueous $\mathrm{NaHCO}_{3}$, and the phases are separated. The aqueous phase is extracted with $\mathrm{CH}_{2} \mathrm{Cl}_{2}(3 \times 20 \mathrm{~mL})$. The combined organic phases are washed with brine, dried over $\mathrm{Na}_{2} \mathrm{SO}_{4}$, and concentrated under reduced pressure. The residue is purified by flash column chromatography on silica gel using cyclohexane/ethyl acetate (40/1) as eluent to afford 2-halo-2'-phenylthio-1,1'-binaphthyls as solids.

\subsection{1 rac-2-Bromo-2'-phenylthio-1,1'-binaphthyl (rac-9)}

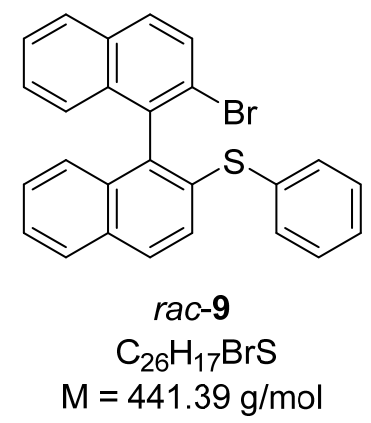

Prepared according to GP1 from rac-2,2'-dibromo-1,1'-binaphthyl (rac-8, $300 \mathrm{mg}, 0.728 \mathrm{mmol}$, 1.00 equiv) and diphenyl disulfide (191 mg, $0.874 \mathrm{mmol}, 1.20$ equiv) in 95\% yield (306 mg, $0.693 \mathrm{mmol}$ ). The product rac-9 was obtained as a white solid after flash column chromatography on silica gel using cyclohexane/ethyl acetate (40/1) as eluent. $\boldsymbol{R}_{\mathbf{f}}=0.37$ (cyclohexane/ethyl acetate 40/1). m.p.: $60{ }^{\circ} \mathrm{C}$ (cyclohexane). GLC (HP-5): $t_{R}=45.6 \mathrm{~min}$. IR (ATR): $\tilde{\mathrm{v}}=3049$ (w), $2920(\mathrm{w})$, $1576(m), 1498(m), 1472(m), 1437(m), 1304(w), 1254(w), 1108(w), 1021(w), 942(w), 834(w)$, 
804 (s), 737 (s), 688 (s) $\mathrm{cm}^{-1}$. HRMS (APCl) calculated for $\mathrm{C}_{26} \mathrm{H}_{17} \mathrm{BrS}$ [M] ${ }^{+}:$440.0229; found: 440.0230. ${ }^{1} \mathrm{H}$ NMR $\left(500 \mathrm{MHz}, \mathrm{CDCl}_{3}\right): \delta 7.08(\mathrm{~d}, J=8.5 \mathrm{~Hz}, 1 \mathrm{H}), 7.18(\mathrm{~d}, J=8.5 \mathrm{~Hz}, 1 \mathrm{H}), 7.25-$ $7.32(\mathrm{~m}, 5 \mathrm{H}), 7.37(\mathrm{~d}, J=8.7 \mathrm{~Hz}, 1 \mathrm{H}), 7.40-7.41(\mathrm{~m}, 2 \mathrm{H}), 7.45$ (ddd, $J=8.0 \mathrm{~Hz}, J=7.0 \mathrm{~Hz}, J=1.0$ $\mathrm{Hz}, 1 \mathrm{H}), 7.50$ (ddd, $J=8.0 \mathrm{~Hz}, J=7.0 \mathrm{~Hz}, J=1.0 \mathrm{~Hz}, 1 \mathrm{H}), 7.83-7.88(\mathrm{~m}, 4 \mathrm{H}), 7.93(\mathrm{~d}, J=8.2 \mathrm{~Hz}$, 1H). ${ }^{13} \mathrm{C}$ NMR $\left(126 \mathrm{MHz}, \mathrm{CDCl}_{3}\right): \delta 123.3,125.6,126.1,126.2,126.4,127.2,127.4,127.7,127.8$, $128.3,128.4,129.1,129.3,129.9,130.0,132.2$, 132.6, 132.7, 133.0, 134.1, 134.9, 135.6, 136.0, 136.5. Elemental analysis was not performed. The analytical and spectroscopic data are in accordance with those reported. ${ }^{[\mathrm{S} 2]}$

\subsection{2 (S)-2-lodo-2'-phenylthio-1,1'-binaphthyl [(S)-7]}

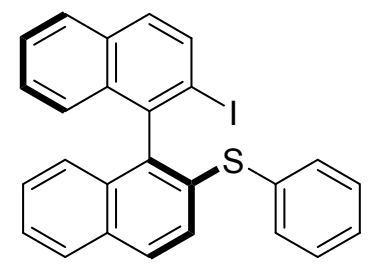

(S)-7

$$
\mathrm{C}_{26} \mathrm{H}_{17} \mathrm{IS}
$$

$\mathrm{M}=488.39 \mathrm{~g} / \mathrm{mol}$

Prepared according to GP1 from (S)-2,2'-diiodo-1,1'-binaphthyl [(S)-6, $670 \mathrm{mg}, 1.32 \mathrm{mmol}$, 1.00 equiv] and diphenyl disulfide (346 mg, $1.58 \mathrm{mmol}, 1.20$ equiv). Desired (S)-7 was obtained together with the disubstituted derivative in a ratio of $2: 1\left(300 \mathrm{mg}, 46 \%\right.$, determined by ${ }^{1} \mathrm{H}$ NMR analysis) as a pale yellow solid after flash column chromatography on silica gel using cyclohexane/ethyl acetate (40/1) as eluent.

\subsection{2 (R)-2-lodo-2'-phenylthio-1,1'-binaphthyl [(R)-7]}

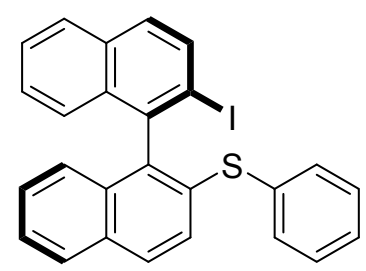

$(R)-7$

$\mathrm{C}_{26} \mathrm{H}_{17} \mathrm{IS}$

$\mathrm{M}=488.39 \mathrm{~g} / \mathrm{mol}$

Prepared according to GP2 from $(R)-2,2$ '-diiodo-1,1'-binaphthyl $[(R)-6,190 \mathrm{mg}, 0.375 \mathrm{mmol}$, 1.00 equiv] and diphenyl disulfide (98 mg, $0.45 \mathrm{mmol}, 1.2$ equiv). Desired $(R)-7$ was obtained 
together with the disubstituted derivative in a ratio of $2: 1$ (82 $\mathrm{mg}, 45 \%$, determined by ${ }^{1} \mathrm{H}$ NMR analysis) as a pale yellow solid after flash column chromatography on silica gel using cyclohexane/ethyl acetate (40/1) as eluent.

\subsection{General Procedure for the Synthesis of the Diastereomeric Hydrosilanes (GP2)}

To a solution of aryl halides rac-9 (1.00 equiv) or $(S)-/(R)-7$ (1.40 equiv) in THF $(0.03 \mathrm{M})$ cooled to $-78^{\circ} \mathrm{C}$ is added $n \mathrm{BuLi}$ (1.10 or 1.54 equiv) dropwise, and the resulting mixture is maintained at $-78^{\circ} \mathrm{C}$ for $1 \mathrm{~h}$. (S)-10 (1.00 equiv) dissolved in THF $(0.11 \mathrm{M})$ is quickly added, and the mixture is subsequently allowed to warm to room temperature, followed by stirring overnight. The reaction is quenched by the addition of water, and the phases are separated. The aqueous phase is extracted with $\mathrm{CH}_{2} \mathrm{Cl}_{2}(3 \times 20 \mathrm{~mL})$, the combined organic phases are washed with brine, and dried over $\mathrm{Na}_{2} \mathrm{SO}_{4}$. The solvents are removed under reduced pressure. The residue is purified by flash column chromatography on silica gel using cyclohexane/ $\mathrm{CH}_{2} \mathrm{Cl}_{2}(10 / 1)$ as eluent to afford hydrosilanes $(S, R S)-\mathbf{1 1},(S, S)-\mathbf{1 1}$, and $(S, R)$-11 as white solids.

\subsection{1 (4RS,11bS)-4-(2'-Phenylthio-[1,1'-binaphthalen]-2-yl)-4,5-dihydro-3H-dinaphtho[2,1-c:1',2'-} e]silepine $[(S, R S)-11]$

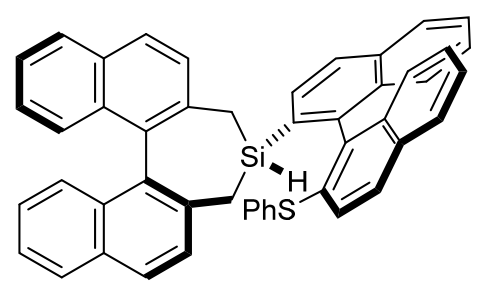

$(S, R S)-11$

$$
\mathrm{C}_{48} \mathrm{H}_{34} \mathrm{SSi}
$$

$M=670.95 \mathrm{~g} / \mathrm{mol}$

Prepared according to GP2 from aryl bromide rac-9 (142 mg, $0.322 \mathrm{mmol}, 1.00$ equiv) and (S)-10 (100 mg, $0.322 \mathrm{mmol}, 1.00$ equiv) in 57\% yield. The hydrosilane (S,RS)-11 was obtained as a white solid after flash column chromatography on silica gel using cyclohexane $/ \mathrm{CH}_{2} \mathrm{Cl}_{2}(10 / 1)$ as eluent. $\boldsymbol{R}_{\mathbf{f}}$ $=0.28$ (cyclohexane/ $\mathrm{CH}_{2} \mathrm{Cl}_{2}$ 10/1). m.p.: $168-170{ }^{\circ} \mathrm{C}$ (benzene). IR (ATR): $\tilde{\mathrm{v}}=3039(\mathrm{w}), 2140(\mathrm{w})$, $1579(w), 1498$ (w), $1473(w), 1309$ (w), 1238 (w), 1137 (w), 1021 (w), 809 (s), 741 (s), $689(\mathrm{~m})$, $673(\mathrm{~m}) \mathrm{cm}^{-1}$. HRMS (APCl) calculated for $\mathrm{C}_{48} \mathrm{H}_{34} \mathrm{SSi}[\mathrm{M}]^{+}: 670.2145$; found: $670.2172 .{ }^{1} \mathbf{H}$ NMR $\left(500 \mathrm{MHz}, \mathrm{CDCl}_{3}\right): \delta 1.57(\mathrm{~d}, J=13.8 \mathrm{~Hz}, 1 \mathrm{H}), 1.70-1.76(\mathrm{~m}, 2 \mathrm{H}), 2.06-2.14(\mathrm{~m}, 3 \mathrm{H}), 2.35-2.39$ $(\mathrm{m}, 1 \mathrm{H}), 2.46(\mathrm{~d}, J=13.5 \mathrm{~Hz}, 1 \mathrm{H}), 3.86\left(\mathrm{~m}_{\mathrm{c}}, 1 \mathrm{H}\right), 4.04\left(\mathrm{~m}_{\mathrm{c}}, 1 \mathrm{H}\right), 6.94(\mathrm{~d}, J=8.5 \mathrm{~Hz}, 1 \mathrm{H}), 7.00(\mathrm{~d}, J=$ 
8.5 Hz, 1H), $7.05\left(\mathrm{~m}_{\mathrm{c}}, 1 \mathrm{H}\right), 7.09-7.34(\mathrm{~m}, 28 \mathrm{H}), 7.36-7.43(\mathrm{~m}, 9 \mathrm{H}), 7.47-7.52(\mathrm{~m}, 3 \mathrm{H}), 7.70(\mathrm{~d}, J=$ $8.3 \mathrm{~Hz}, 1 \mathrm{H}), 7.75-7.93(\mathrm{~m}, 16 \mathrm{H}) .{ }^{13} \mathrm{C}$ NMR $\left(126 \mathrm{MHz}, \mathrm{CDCl}_{3}\right): \delta 19.8,20.0,20.0,20.4,124.5$, $124.5,125.7,125.8,125.9,125.9,126.0,126.2$, 126.2, 126.3, 126.4, 126.5, 126.7, 127.0, 127.0, $127.0,127.4,127.4,127.7,127.7,127.9,128.0,128.0,128.1,128.1,128.1,128.2,128.3,128.3$, $128.5,128.7,128.8,128.9,129.2,129.4,129.5,130.8,131.6,131.9,132.0,132.0,132.1,132.4$, $132.5,132.6,132.6,132.7,132.7,132.8,133.0,133.3$, 133.5, 134.0, 134.1, 134.4, 134.5, 135.7, $135.8,136.1,136.1,136.1,136.2,136.3,136.5,143.8,143.9 .{ }^{29}$ Si DEPT $\left(99 \mathrm{MHz}, \mathrm{CDCl}_{3}\right): \delta-7.69$, -7.67 . Elemental analysis was not performed.

\subsection{2 (4S,11bS)-4-(2'-Phenylthio-[1,1'-binaphthalen]-2-yl)-4,5-dihydro-3H-dinaphtho[2,1-c:1',2'-} e]silepine $[(S, S)-11]$

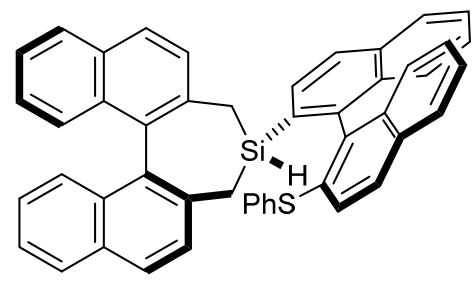

$$
\begin{gathered}
(S, S)-11 \\
\mathrm{C}_{48} \mathrm{H}_{34} \mathrm{SSi} \\
\mathrm{M}=670.95 \mathrm{~g} / \mathrm{mol}
\end{gathered}
$$

Prepared according to GP2 from contaminated aryl iodide (S)-7 (377 mg, $0.772 \mathrm{mmol}, 1.40$ equiv) and (S)-10 (171 mg, $0.551 \mathrm{mmol}, 1.00$ equiv) in $37 \%$ yield (128 mg, $0.191 \mathrm{mmol}$ ). The hydrosilane $(S, S)$-11 was obtained as a white solid after flash column chromatography on silica gel using cyclohexane $/ \mathrm{CH}_{2} \mathrm{Cl}_{2}(10 / 1)$ as eluent. $\boldsymbol{R}_{\mathrm{f}}=0.13$ (cyclohexane $/ \mathrm{CH}_{2} \mathrm{Cl}_{2}$ 10/1). m.p.: $168-170{ }^{\circ} \mathrm{C}$ (benzene). IR (ATR): $\tilde{v}=3057(w), 2909$ (w), $2166(w), 2144$ (w), $1579(w), 1499$ (w), 1239 (w), 1135 (br w), 1023 (w), $830(\mathrm{~m}), 808(\mathrm{~m}), 740(\mathrm{~s}), 690(\mathrm{~m}), 670(\mathrm{~m}) \mathrm{cm}^{-1}$. HRMS (APCl) calculated for $\mathrm{C}_{48} \mathrm{H}_{34} \mathrm{SSi}[\mathrm{M}]^{+}:$: 670.2145; found: $670.2136 .{ }^{1} \mathrm{H}$ NMR $\left(500 \mathrm{MHz}, \mathrm{CDCl}_{3}\right): \delta 1.73(\mathrm{~d}, J=12.6 \mathrm{~Hz}$, $1 \mathrm{H}), 2.08-2.13(\mathrm{~m}, 2 \mathrm{H}), 2.48(\mathrm{~d}, J=13.6 \mathrm{~Hz}, 1 \mathrm{H}), 3.88(\mathrm{~d}, J=7.5 \mathrm{~Hz}, 1 \mathrm{H}), 6.96(\mathrm{~d}, J=8.5 \mathrm{~Hz}, 1 \mathrm{H})$, 7.05-7.19 (m, 7H), 7.22-7.33 (m, 6H), 7.38-7.40 (m, 4H), 7.48-7.50 (m, 2H), $7.71(\mathrm{~d}, J=8.4 \mathrm{~Hz}$, 1H), 7.77-7.87 (m, 6H), 7.90-7.92 (m, 2H). ${ }^{13} \mathrm{C}$ NMR (126 MHz, $\left.\mathrm{CDCl}_{3}\right): \delta 20.0,20.4,124.5,124.5$, $125.7,125.9,126.0,126.2,126.4,126.5,126.6,127.0,127.0,127.3,127.4,127.7,128.0,128.0$, $128.1,128.1,128.2,128.4,128.5,128.7,128.9,128.9,129.3,129.5,130.8,131.6,132.0,132.1$, $132.4,132.5,132.7,132.7,132.8,133.0,133.5,134.1,134.5,135.7,136.1,136.2,136.3,143.8$. ${ }^{29}$ Si DEPT (99 MHz, $\left.\mathrm{CDCl}_{3}\right): \delta-7.67$. Anal. Calcd for $\mathrm{C}_{48} \mathrm{H}_{34} \mathrm{SSi}$ C, 85.93; $\mathrm{H}, 5.11 ; \mathrm{S}, 4.78$. Found: C, 85.78; H, 5.42; S, 4.52. 
2.2.3 (4R,11bS)-4-(2'-Phenylthio-[1,1'-binaphthalen]-2-yl)-4,5-dihydro-3H-dinaphtho[2,1-c:1',2'e]silepine $[(S, R)-11]$

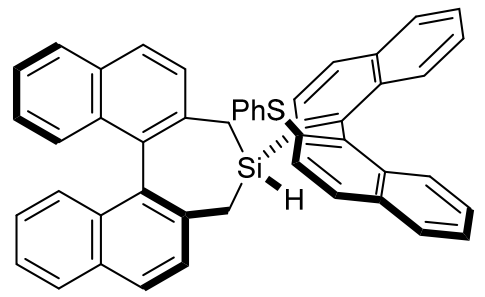

$(S, R)-11$

$\mathrm{C}_{48} \mathrm{H}_{34} \mathrm{SSi}$

$M=670.95 \mathrm{~g} / \mathrm{mol}$

Prepared according to GP2 from contaminated aryl iodide $(R)-7$ (126 mg, $0.252 \mathrm{mmol}, 1.40$ equiv) and (S)-10 (57 mg, $0.18 \mathrm{mmol}, 1.0$ equiv) in $32 \%$ yield (32 mg, $0.048 \mathrm{mmol}$ ). The hydrosilane $(S, R)$-11 was obtained as a white solid after flash column chromatography on silica gel using cyclohexane/ $\mathrm{CH}_{2} \mathrm{Cl}_{2}$ (10/1) as eluent. $\boldsymbol{R}_{\mathrm{f}}=0.41$ (cyclohexane $/ \mathrm{CH}_{2} \mathrm{Cl}_{2}$ 10/1). m.p.: $168-170{ }^{\circ} \mathrm{C}$ (benzene). IR (ATR): $\tilde{v}=3054(w), 2908$ (w), $2140(w), 1579(w), 1499(w), 1475(w), 1240(w)$, 1141 (br w), $1023(\mathrm{w}), 830(\mathrm{~m}), 809(\mathrm{~m}), 740(\mathrm{~s}), 690(\mathrm{~m}), 670(\mathrm{~m}) \mathrm{cm}^{-1}$. HRMS (APCl) calculated for $\mathrm{C}_{48} \mathrm{H}_{34} \mathrm{SSi}[\mathrm{M}]^{+}:$: 670.2145; found: $670.2136 .{ }^{1} \mathrm{H}$ NMR $\left(500 \mathrm{MHz}, \mathrm{CDCl}_{3}\right): \delta 1.57(\mathrm{~d}, J=13.6 \mathrm{~Hz}$, $1 \mathrm{H}), 1.74-1.77(\mathrm{~m}, 1 \mathrm{H}), 2.12-2.15(\mathrm{~m}, 1 \mathrm{H}), 2.36-2.40(\mathrm{~m}, 1 \mathrm{H}), 4.04-4.05(\mathrm{~m}, 1 \mathrm{H}), 7.01(\mathrm{~d}, J=8.6$ $\mathrm{Hz}, 1 \mathrm{H}), 7.10-7.22(\mathrm{~m}, 5 \mathrm{H}), 7.24-7.30(\mathrm{~m}, 5 \mathrm{H}), 7.31-7.35(\mathrm{~m}, 5 \mathrm{H}), 7.36-7.44(\mathrm{~m}, 4 \mathrm{H}), 7.51\left(\mathrm{~m}_{\mathrm{c}}\right.$, 1H), 7.76-7.86 (m, 6H), 7.89-7.92 (m, 2H). ${ }^{13} \mathrm{C}$ NMR (126 MHz, CDCl $)$ : $\delta 19.8,20.0,124.5,124.5$, $125.8,125.9,125.9,126.2,126.3,126.4,126.5,126.6,126.7,127.0,127.0,127.4,127.7,127.9$, $128.0,128.1,128.1,128.2,128.3,128.3,128.5,128.8,129.4,130.8,131.9,132.0,132.5,132.6$, $132.7,132.7,133.0,133.3,134.0,134.4,134.5,135.8,136.1,136.2,136.5,143.9 .{ }^{29}$ Si DEPT (99 $\mathrm{MHz}, \mathrm{CDCl}_{3}$ ): $\delta-7.69$. Elemental analysis was not performed.

\section{General Procedure for the Generation of Intramolecularly Sulfur-Stabilized Silicon Cations (GP3)}

In a glovebox, a solution of the indicated hydrosilane (1.00 equiv) in $1,2-\mathrm{Cl}_{2} \mathrm{C}_{6} \mathrm{D}_{4}(0.4 \mathrm{~mL})$ is added to a solution of $\left[\mathrm{Ph}_{3} \mathrm{C}\right]^{+}\left[\mathrm{B}\left(\mathrm{C}_{6} \mathrm{~F}_{5}\right)_{4}\right]^{-}\left(1.00\right.$ equiv) in $1,2-\mathrm{Cl}_{2} \mathrm{C}_{6} \mathrm{D}_{4}(0.2 \mathrm{~mL})$ in a $8 \mathrm{~mL}$-vial. The resulting mixture is stirred for $5 \mathrm{~min}$, transferred to an NMR tube, and directly subjected to NMR spectroscopic analysis. 
3.1 (4RS,11bS)-4-(2'-Phenylthio-[1,1'-binaphthalen]-2-yl)-4,5-dihydro-3H-dinaphtho[ 2,1-c:1',2'-e]silepinylium Tetrakis(pentafluorophenyl)borate [(S,RS)-5]

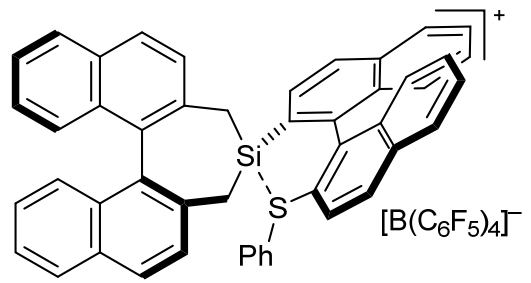

$(S, R S)-5$

$\mathrm{C}_{72} \mathrm{H}_{33} \mathrm{BF}_{20} \mathrm{SSi}$

$M=1348.98 \mathrm{~g} / \mathrm{mol}$

Prepared according to GP3 from (4RS,11bS)-4-(2'-phenylthio-[1,1'-binaphthalen]-2-yl)-4,5-dihydro$3 H$-dinaphtho[2,1-c:1',2'-e]silepine $\quad[(S, R S)-11, \quad 33.5 \mathrm{mg}, \quad 50.0 \mu \mathrm{mol}, \quad 1.00$ equiv) and $\left[\mathrm{Ph}_{3} \mathrm{C}\right]^{+}\left[\mathrm{B}\left(\mathrm{C}_{6} \mathrm{~F}_{5}\right)_{4}\right]^{-}\left(46.1 \mathrm{mg}, 50.0 \mu \mathrm{mol}, 1.00\right.$ equiv). ${ }^{1} \mathrm{H}$ NMR $\left(500 \mathrm{MHz}, 1,2-\mathrm{Cl}_{2} \mathrm{C}_{6} \mathrm{D}_{4}\right): \delta 1.31(\mathrm{~d}, \mathrm{~J}=$ $15.2 \mathrm{~Hz}, 1 \mathrm{H}), 1.40-1.43(\mathrm{~m}, 1 \mathrm{H}), 2.12(\mathrm{~d}, J=15.0 \mathrm{~Hz}, 1 \mathrm{H}), 2.27(\mathrm{~d}, J=13.8 \mathrm{~Hz}, 1 \mathrm{H}), 2.56-2.59(\mathrm{~m}$, $1 \mathrm{H}), 2.89-2.95(\mathrm{~m}, 3 \mathrm{H}), 6.45-6.48(\mathrm{~m}, 2 \mathrm{H}), 6.62-6.70(\mathrm{~m}, 3 \mathrm{H}), 6.73-6.79(\mathrm{~m}, 2 \mathrm{H}), 6.92-7.20(\mathrm{~m}$, $8 \mathrm{H}),{ }^{*} 7.29-7.53(\mathrm{~m}, 20 \mathrm{H}), 7.55-8.02(\mathrm{~m}, 20 \mathrm{H}), 8.05(\mathrm{~d}, J=8.5 \mathrm{~Hz}, 1 \mathrm{H}), 8.12(\mathrm{~d}, J=8.5 \mathrm{~Hz}, 1 \mathrm{H})$, $8.32(\mathrm{~d}, J=8.5 \mathrm{~Hz}, 1 \mathrm{H}) .{ }^{13} \mathrm{C}$ NMR $\left(126 \mathrm{MHz}, 1,2-\mathrm{Cl}_{2} \mathrm{C}_{6} \mathrm{D}_{4}\right): \delta-4.0,-3.0,14.3,22.7,114.6,120.0$, 123.7, 124.8 (br m), 125.7, 125.8, 126.0, 126.1, 126.2, 126.9, 127.6, 127.8, 127.9, 128.1, 128.2, 128.3, 128.7, 128.8, 128.9, 129.1, 129.2, 129.4, 130.4, 130.6, 130.8, 131.4, 131.8, 132.2, 132.8, 132.9, 133.2, 134.0, 134.1, 136.4, 136.7 (d, $J=240 \mathrm{~Hz}), 138.7$ (d, $J=250 \mathrm{~Hz}), 139.4,140.1,142.4$, 143.3, $148.8(\mathrm{~d}, J=242 \mathrm{~Hz}) .{ }^{11} \mathrm{~B}$ NMR $\left(160 \mathrm{MHz}, 1,2-\mathrm{Cl}_{2} \mathrm{C}_{6} \mathrm{D}_{4}\right): \delta-16.0 .{ }^{19} \mathrm{~F}$ NMR $(470 \mathrm{MHz}, 1,2-$ $\left.\mathrm{Cl}_{2} \mathrm{C}_{6} \mathrm{D}_{4}\right): \delta-165.8,-161.9,-131.5 .{ }^{1} \mathrm{H},{ }^{29} \mathrm{Si}$ HMQC NMR (500/99 MHz, $\left.1,2-\mathrm{Cl}_{2} \mathrm{C}_{6} \mathrm{D}_{4}\right): \delta 39.7,32.8$, 7.5, $-13.5 \mathrm{ppm}$.

*Signal overlapping of $1,2-\mathrm{Cl}_{2} \mathrm{C}_{6} \mathrm{D}_{4}$ and $\mathrm{Ph}_{3} \mathrm{CH}$ resonances (8H expected). 
3.2 (4S,11bS)-4-(2'-Phenylthio-[1,1'-binaphthalen]-2-yl)-4,5-dihydro-3H-dinaphtho[ 2,1-c:1',2'-e]silepinylium Tetrakis(pentafluorophenyl)borate $[(S, S)-5]$

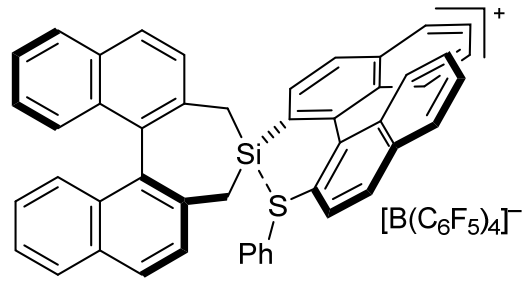

$(S, S)-5$

$\mathrm{C}_{72} \mathrm{H}_{33} \mathrm{BF}_{20} \mathrm{SSi}$

$\mathrm{M}=1348.98 \mathrm{~g} / \mathrm{mol}$

Prepared according to GP3 from (4S,11bS)-4-(2'-phenylthio-[1,1'-binaphthalen]-2-yl)-4,5-dihydro$3 H$-dinaphtho[2,1-c:1',2'-e]silepine $\quad[(S, S)-11, \quad 33.5 \quad \mathrm{mg}, \quad 50.0 \mu \mathrm{mol}, \quad 1.00$ equiv) and $\left[\mathrm{Ph}_{3} \mathrm{C}\right]^{+}\left[\mathrm{B}\left(\mathrm{C}_{6} \mathrm{~F}_{5}\right)_{4}\right]^{-}\left(46.1 \mathrm{mg}, 50.0 \mu \mathrm{mol}, 1.00\right.$ equiv). ${ }^{1} \mathrm{H}$ NMR $\left(500 \mathrm{MHz}, 1,2-\mathrm{Cl}_{2} \mathrm{C}_{6} \mathrm{D}_{4}\right): \delta 1.31(\mathrm{~d}, J=$ $15.4 \mathrm{~Hz}, 1 \mathrm{H}), 2.11(\mathrm{~d}, J=14.6 \mathrm{~Hz}, 1 \mathrm{H}), 2.91\left(\mathrm{~m}_{\mathrm{c}}, 2 \mathrm{H}\right), 6.46-6.47(\mathrm{~m}, 2 \mathrm{H}), 6.64-6.66(\mathrm{~m}, 2 \mathrm{H}), 6.74-$ $6.75(\mathrm{~m}, 1 \mathrm{H}), 7.03-7.27(\mathrm{~m}, 4 \mathrm{H}),{ }^{*} 7.32-7.40(\mathrm{~m}, 5 \mathrm{H}), 7.46-7.51(\mathrm{~m}, 2 \mathrm{H}), 7.59\left(\mathrm{~m}_{\mathrm{c}}, 1 \mathrm{H}\right), 7.66-8.12$ $(\mathrm{m}, 11 \mathrm{H}), 8.32(\mathrm{~d}, J=8.4 \mathrm{~Hz}, 1 \mathrm{H}) .{ }^{13} \mathrm{C}$ NMR $\left(126 \mathrm{MHz}, 1,2-\mathrm{Cl}_{2} \mathrm{C}_{6} \mathrm{D}_{4}\right): \delta$ 19.3, 19.9, 114.0, 120.3, 122.5, 124.8 (br m), 125.8, 126.3, 126.6, 128.1, 128.2, 128.4, 128.7, 128.8, 129.0, 129.0, 129.1, $129.2,129.3,129.3,129.5,130.6,131.0,131.2,131.6,132.2,132.8,132.9,133.0,133.1$, 133.2, 133.4, 133.6, 134.0, 136.8 (d, $J=247 \mathrm{~Hz}), 137.0,138.7$ (d, $J=233 \mathrm{~Hz}), 140.2,140.7,142.4,143.3$, $148.9(\mathrm{~d}, J=236 \mathrm{~Hz}) .{ }^{11} \mathrm{~B}$ NMR $\left(160 \mathrm{MHz}, 1,2-\mathrm{Cl}_{2} \mathrm{C}_{6} \mathrm{D}_{4}\right): \delta-15.9 .{ }^{19} \mathrm{~F}$ NMR $\left(470 \mathrm{MHz}, 1,2-\mathrm{Cl}_{2} \mathrm{C}_{6} \mathrm{D}_{4}\right)$ : $\delta-165.8,-162.0,-131.4 .{ }^{29}$ Si DEPT NMR (99 MHz, 1,2-Cl $\left.{ }_{2} \mathrm{C}_{6} \mathrm{D}_{4}\right): \delta 39.9 \mathrm{ppm}$.

*Signal overlapping of $1,2-\mathrm{Cl}_{2} \mathrm{C}_{6} \mathrm{D}_{4}$ and $\mathrm{Ph}_{3} \mathrm{CH}$ resonances (4H expected). 
3.3 (4R,11bS)-4-(2'-Phenylthio-[1,1'-binaphthalen]-2-yl)-4,5-dihydro-3H-dinaphtho[ 2,1-c:1',2'-e]silepinylium Tetrakis(pentafluorophenyl)borate $[(S, R)-5]$

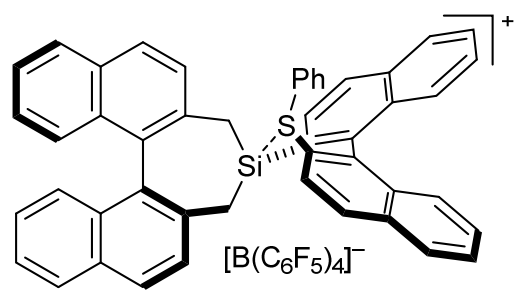

$(S, R)-5$

$\mathrm{C}_{72} \mathrm{H}_{33} \mathrm{BF}_{20} \mathrm{SSi}$

$M=1348.98 \mathrm{~g} / \mathrm{mol}$

Prepared according to GP3 from (4R,11bS)-4-(2'-phenylthio-[1,1'-binaphthalen]-2-yl)-4,5-dihydro$3 H$-dinaphtho[2,1-c:1',2'-e]silepine $\quad[(S, R)-11, \quad 33.5 \quad \mathrm{mg}, \quad 50.0 \mu \mathrm{mol}, \quad 1.00$ equiv) and $\left[\mathrm{Ph}_{3} \mathrm{C}\right]^{+}\left[\mathrm{B}\left(\mathrm{C}_{6} \mathrm{~F}_{5}\right)_{4}\right]^{-}\left(46.1 \mathrm{mg}, 50.0 \mu \mathrm{mol}, 1.00\right.$ equiv). ${ }^{1} \mathrm{H}$ NMR $\left(500 \mathrm{MHz}, 1,2-\mathrm{Cl}_{2} \mathrm{C}_{6} \mathrm{D}_{4}\right): \delta 1.41-1.43$ (m, 1H), 2.27 (d, J = 14.2 Hz, 1H), 2.60-2.63 (m, 1H), 2.93 (d, J=15.2 Hz, 1H), 6.67 (d, J = 8.3 Hz, $1 \mathrm{H}), 6.91-6.93(\mathrm{~m}, 4 \mathrm{H}), 7.06-7.19(\mathrm{~m}, 4 \mathrm{H}),{ }^{*} 7.31-7.90(\mathrm{~m}, 19 \mathrm{H}), 7.96-7.97(\mathrm{~m}, 1 \mathrm{H}) .{ }^{13} \mathrm{C}$ NMR $(126$ $\left.\mathrm{MHz}, 1,2-\mathrm{Cl}_{2} \mathrm{C}_{6} \mathrm{D}_{4}\right): \delta 18.3,20.3,124.7$ (br m), 125.9, 126.0, 126.1, 126.5, 126.6, 126.6, 127.5, $127.6,128.1,128.5,128.7,128.8,128.9,129.0,129.1,129.2,130.3,130.8,131.5,132.0,132.4$, 132.5, 132.9, 133.3, 135.9, 136.5 (d, $J=244 \mathrm{~Hz}$ ), 137.5 (d, $J=240 \mathrm{~Hz}$ ), 140.2, 140.9, 142.2, 143.0, $148.6(\mathrm{~d}, J=240 \mathrm{~Hz}) .{ }^{11} \mathrm{~B}$ NMR $\left(160 \mathrm{MHz}, 1,2-\mathrm{Cl}_{2} \mathrm{C}_{6} \mathrm{D}_{4}\right): \delta-16.2 .{ }^{19} \mathrm{~F}$ NMR $\left(470 \mathrm{MHz}, 1,2-\mathrm{Cl}_{2} \mathrm{C}_{6} \mathrm{D}_{4}\right)$ : $\left.\delta-166.0,-162.2,-131.7 .{ }^{1} \mathrm{H}^{29}{ }^{29} \mathrm{Si} \mathrm{HMQC} \mathrm{NMR} \mathrm{(500/99} \mathrm{MHz,} 1,2-\mathrm{Cl}_{2} \mathrm{C}_{6} \mathrm{D}_{4}\right): \delta 32.8,7.5,-14.5 \mathrm{ppm}$. *Signal overlapping of $1,2-\mathrm{Cl}_{2} \mathrm{C}_{6} \mathrm{D}_{4}$ and $\mathrm{Ph}_{3} \mathrm{CH}$ resonances (4H expected). 
3.4 (4R,11bS)-4-(2'-Phenylthio-[1,1'-binaphthalen]-2-yl)-4,5-dihydro-3H-dinaphtho[ 2,1-c:1',2'-e]silepinylium Tetrakis(pentafluorophenyl)borate Benzophenone Adduct $\left[(S, R)-5 \cdot O=\mathrm{CPh}_{2}\right]$

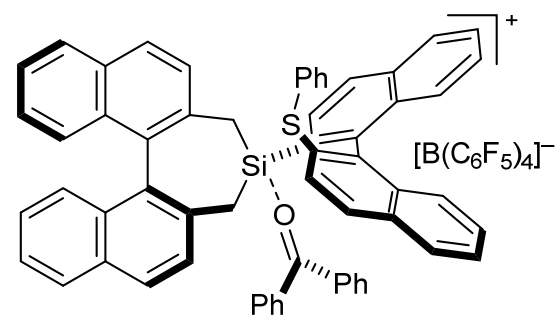

$(S, R)-\mathbf{x x}$

$\mathrm{C}_{85} \mathrm{H}_{43} \mathrm{BF}_{20} \mathrm{OSSi}$

$\mathrm{M}=1531.20 \mathrm{~g} / \mathrm{mol}$

In a glovebox, a solution of (4R,11bS)-4-(2'-phenylthio-[1,1'-binaphthalen]-2-yl)-4,5-dihydro-3Hdinaphtho[2,1-c:1',2'-e]silepine $\left[(S, R)-11,33.5 \mathrm{mg}, 50.0 \mu \mathrm{mol}, 1.00\right.$ equiv) in $1,2-\mathrm{Cl}_{2} \mathrm{C}_{6} \mathrm{D}_{4}(0.4 \mathrm{~mL})$ was added to a solution of $\left[\mathrm{Ph}_{3} \mathrm{C}\right]^{+}\left[\mathrm{B}\left(\mathrm{C}_{6} \mathrm{~F}_{5}\right)_{4}\right]^{-}\left(46.1 \mathrm{mg}, 50.0 \mu \mathrm{mol}, 1.00\right.$ equiv) in $1,2-\mathrm{Cl}_{2} \mathrm{C}_{6} \mathrm{D}_{4}$ $(0.2 \mathrm{~mL})$ in an $8 \mathrm{~mL}$-vial. After stirring for $5 \mathrm{~min}$, a solution of benzophenone $(9.23 \mathrm{mg}, 50.0 \mu \mathrm{mol}$, 1.00 equiv) was added, the mixture transferred to an NMR tube, and directly subjected to NMR spectroscopic analysis. ${ }^{1} \mathrm{H}$ NMR $\left(500 \mathrm{MHz}, 1,2-\mathrm{Cl}_{2} \mathrm{C}_{6} \mathrm{D}_{4}\right): \delta 1.78\left(\mathrm{~m}_{\mathrm{c}}, 2 \mathrm{H}\right), 2.33(\mathrm{~d}, J=13.9 \mathrm{~Hz}, 1 \mathrm{H})$, $2.69(\mathrm{~d}, J=14.8 \mathrm{~Hz}, 1 \mathrm{H}), 6.62\left(\mathrm{~m}_{\mathrm{c}}, 2 \mathrm{H}\right), 6.71\left(\mathrm{~m}_{\mathrm{c}}, 1 \mathrm{H}\right), 6.81\left(\mathrm{~m}_{\mathrm{c}}, 1 \mathrm{H}\right), 6.93-7.38(\mathrm{~m}, 29 \mathrm{H})^{\star}, 7.60$ $7.72(\mathrm{~m}, 9 \mathrm{H}), 7.81-7.91(\mathrm{~m}, 3 \mathrm{H}), 8.11\left(\mathrm{~m}_{\mathrm{c}}, 1 \mathrm{H}\right) .{ }^{13} \mathrm{C} \mathrm{NMR}\left(126 \mathrm{MHz}, 1,2-\mathrm{Cl}_{2} \mathrm{C}_{6} \mathrm{D}_{4}\right): \delta 22.0,22.5$, $124.9,125.0,125.7,126.0,126.2,126.3,126.6,127.6,127.7,127.9,128.1,128.5,128.7,128.9$, $129.1,129.1,129.3,130.3,130.5,130.7,131.4,131.5,132.0,132.0,132.9,133.2,133.6,133.9$, 135.7, 136.5 (d, $J=232 \mathrm{~Hz}), 138.7,137.5(\mathrm{~d}, J=240 \mathrm{~Hz}), 141.1,142.2,143.1,146.3,148.6(\mathrm{~d}, J=$ $243 \mathrm{~Hz}) .{ }^{11} \mathrm{~B}$ NMR $\left(160 \mathrm{MHz}, 1,2-\mathrm{Cl}_{2} \mathrm{C}_{6} \mathrm{D}_{4}\right): \delta-16.2 .{ }^{19} \mathrm{~F}$ NMR $\left(470 \mathrm{MHz}, 1,2-\mathrm{Cl}_{2} \mathrm{C}_{6} \mathrm{D}_{4}\right): \delta-166.0,-$ 162.2, -131.7. ${ }^{29}$ Si DEPT NMR $\left(99 \mathrm{MHz}, 1,2-\mathrm{Cl}_{2} \mathrm{C}_{6} \mathrm{D}_{4}\right): \delta 31.7 \mathrm{ppm}$.

*Signal overlapping of $1,2-\mathrm{Cl}_{2} \mathrm{C}_{6} \mathrm{D}_{4}$ and $\mathrm{Ph}_{3} \mathrm{CH}$ resonances (29H expected). 


\section{General Procedure for the Preparation of Chalcones 12-20 by Claisen- Schmidt Condensation (GP4)}

The indicated benzaldehyde or 2-naphthaldehyde $(4.20 \mathrm{mmol})$ is dissolved in methanol or ethanol $(10 \mathrm{~mL})$, and the resulting solution is cooled to $0{ }^{\circ} \mathrm{C}$. Then, aqueous $\mathrm{NaOH}$ solution $(10 \%$ wt., $2.2 \mathrm{~mL})$ is added dropwise, followed by slow addition of the indicated ketone $(4.20 \mathrm{mmol}$, 1.00 equiv). The reaction mixture is allowed to warm to room temperature and stirred overnight. The mixture is diluted with water, the precipitate formed is collected by filtration, washed with water, and (if necessary) purified by flash chromatography on silica gel using cyclohexane/ethyl acetate mixtures as eluent. In the case of oils, the reaction mixture is extracted into $\mathrm{CH}_{2} \mathrm{Cl}_{2}$, and the crude material is purified by flash column chromatography on silica gel.

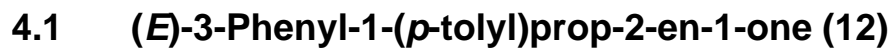

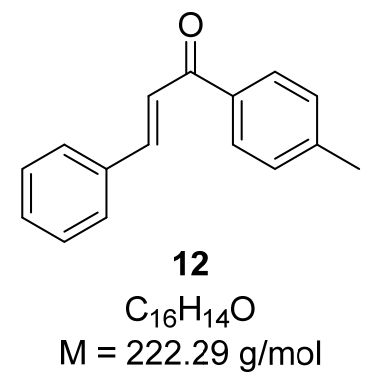

Prepared according to GP4 from benzaldehyde $(0.45 \mathrm{~g}, 0.43 \mathrm{~mL}, 4.2 \mathrm{mmol}, 1.0$ equiv) and 1-(ptolyl)ethan-1-one ( $0.56 \mathrm{~g}, 0.56 \mathrm{~mL}, 4.2 \mathrm{mmol}, 1.0$ equiv) in $40 \%$ yield $(0.37 \mathrm{~g}, 1.6 \mathrm{mmol})$ and with $E: Z>98: 2$. The product was obtained as a yellow solid after flash column chromatography on silica gel using cyclohexane/ethyl acetate (10/1) as eluent. $\boldsymbol{R}_{\mathbf{f}}=0.36$ (cyclohexane/ethyl acetate 10/1). m.p.: $50{ }^{\circ} \mathrm{C}$ (cyclohexane). GLC (HP-5): $t_{\mathrm{R}}=21.8 \mathrm{~min}$. IR (ATR): $\tilde{\mathrm{v}}=3032$ (w), $2054(\mathrm{w}), 1655$ (s), $1597(\mathrm{~s}), 1572(\mathrm{~m}), 1492(\mathrm{~m}), 1448(\mathrm{~m}), 1335(\mathrm{~m}), 1303(\mathrm{~m}), 1224(\mathrm{~m}), 1205(\mathrm{~m}), 1183(\mathrm{~m}), 1031$ (m), $1014(\mathrm{~m}), 972$ (s), 821 (s), 755 (s), 691 (s), 669 (s). HRMS (ESI) calculated for $\mathrm{C}_{16} \mathrm{H}_{13} \mathrm{O}$ [M$\mathrm{H}^{+}:$221.0961; found: 221.0964. ${ }^{1} \mathrm{H}$ NMR (500 MHz, $\left.\mathrm{CDCl}_{3}\right): \delta 2.44(\mathrm{~s}, 3 \mathrm{H}), 7.31$ (d, J = $7.9 \mathrm{~Hz}$, 2H), 7.40-7.43 (m, 3H), $7.54(\mathrm{~d}, J=15.7 \mathrm{~Hz}, 1 \mathrm{H}), 7.64-7.66(\mathrm{~m}, 2 \mathrm{H}), 7.81(\mathrm{~d}, J=15.7 \mathrm{~Hz}, 1 \mathrm{H})$, $7.94(\mathrm{~d}, J=8.2 \mathrm{~Hz}, 2 \mathrm{H}) .{ }^{13} \mathrm{C}$ NMR $\left(126 \mathrm{MHz} \mathrm{CDCl}_{3}\right): \delta 21.8,122.3,128.5,128.8,129.1,129.5$, $130.5,135.2,135.8,143.8,144.5,190.2$. Elemental analysis was not performed. 


\section{2 (E)-1-(4-(tert-Butyl)phenyl)-3-phenylprop-2-en-1-one (13)}

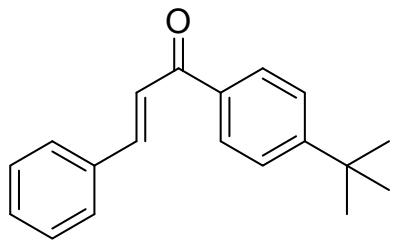

13

$\mathrm{C}_{19} \mathrm{H}_{20} \mathrm{O}$

$\mathrm{M}=264.37 \mathrm{~g} / \mathrm{mol}$

Prepared according to GP4 from benzaldehyde ( $0.45 \mathrm{~g}, 0.43 \mathrm{~mL}, 4.2 \mathrm{mmol}, 1.0$ equiv) and 1-(4(tert-butyl)phenyl)ethan-1-one $(0.74 \mathrm{~g}, 0.77 \mathrm{~mL}, 4.2 \mathrm{mmol}, 1.0$ equiv) in $62 \%$ yield $(0.69 \mathrm{~g}$, $2.6 \mathrm{mmol}$ ) and with $E: Z>98: 2$. The product was obtained as a pale yellow solid after flash column chromatography on silica gel using cyclohexane/ethyl acetate (10/1) as eluent. $\boldsymbol{R}_{\mathbf{f}}=0.41$ (cyclohexane/ethyl acetate 10/1). m.p.: $90^{\circ} \mathrm{C}$ (cyclohexane). GLC (HP-5): $t_{R}=24.1 \mathrm{~min}$. IR (ATR): $\tilde{v}=3067(w), 2964(b r w), 1652(s), 1588(s), 1573(s), 1448(\mathrm{~m}), 1407(w), 1338(\mathrm{~m}), 1223(\mathrm{~m})$, $1187(\mathrm{~m}), 1108(\mathrm{~m}), 980(\mathrm{~m}), 838(\mathrm{~m}), 770(\mathrm{~s}), 742(\mathrm{~s}), 716(\mathrm{~m}), 678(\mathrm{~s}) \mathrm{cm}^{-1}$. HRMS (ESI) calculated for $\mathrm{C}_{19} \mathrm{H}_{19} \mathrm{O}[\mathrm{M}-\mathrm{H}]^{+}: 263.1430$; found: $263.1422 .{ }^{1} \mathrm{H}$ NMR $\left(500 \mathrm{MHz}, \mathrm{CDCl}_{3}\right): \delta 1.37(\mathrm{~s}$, 9H), 7.41-7.45 (m, 3H), 7.52-7.56 (m, 3H), 7.64-7.66 (m, 2H), 7.81 (d, J = $15.8 \mathrm{~Hz}, 1 \mathrm{H}), 7.98\left(\mathrm{~m}_{\mathrm{c}}\right.$, $2 \mathrm{H}) .{ }^{13} \mathrm{C}$ NMR $\left(126 \mathrm{MHz}, \mathrm{CDCl}_{3}\right): \delta 31.2,35.3,122.4,125.7,128.5,128.6,129.1,130.5,135.2$, $135.7,144.5,156.7,190.2$. Elemental analysis was not performed.

\section{3 (E)-1-([1,1'-Biphenyl]-4-yl)-3-phenylprop-2-en-1-one (14)}

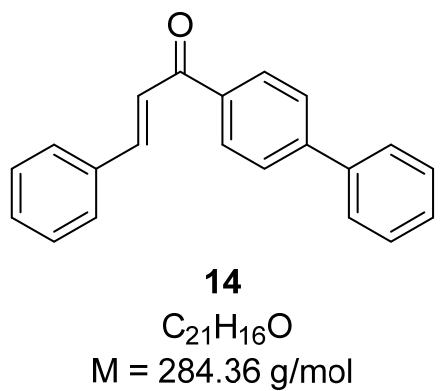

Prepared according to GP4 from benzaldehyde ( $0.45 \mathrm{~g}, 0.43 \mathrm{~mL}, 4.2 \mathrm{mmol}, 1.0$ equiv) and 1-([1,1'biphenyl]-4-yl)ethan-1-one ( $0.84 \mathrm{~g}, 4.2 \mathrm{mmol}, 1.0$ equiv) in $67 \%$ yield $(0.38 \mathrm{~g}, 1.3 \mathrm{mmol})$ and with $E: Z>98: 2$. The product was obtained as a yellow solid after flash column chromatography on silica gel using cyclohexane/ethyl acetate (10/1) as eluent. $\boldsymbol{R}_{\mathbf{f}}=0.34$ (cyclohexane/ethyl acetate 10/1). m.p.: $153-154{ }^{\circ} \mathrm{C}$ (cyclohexane). GLC (HP-5): $t_{R}=18.5 \mathrm{~min}$. IR (ATR): $\tilde{v}=3051(\mathrm{~m}), 3029(\mathrm{w})$, 
$1964(\mathrm{w}), 1655(\mathrm{~m}), 1589(\mathrm{~m}), 1446(\mathrm{~m}), 1400(\mathrm{~m}), 1334(\mathrm{~m}), 1197(\mathrm{w}), 1122(\mathrm{w}), 1033(\mathrm{w}), 982$ (w), 836 (w), 747 (s), 684 (s) cm $\mathrm{cm}^{-1}$. HRMS (ESI) calculated for $\mathrm{C}_{21} \mathrm{H}_{15} \mathrm{O}[\mathrm{M}-\mathrm{H}]^{+}:$283.1117; found: 283.1117. ${ }^{1} \mathrm{H}$ NMR $\left(500 \mathrm{MHz}, \mathrm{CDCl}_{3}\right): \delta 7.40-7.51(\mathrm{~m}, 6 \mathrm{H}), 7.59(\mathrm{~d}, \mathrm{~J}=15.7 \mathrm{~Hz}, 1 \mathrm{H}), 7.65-7.69$ $(\mathrm{m}, 4 \mathrm{H}), 7.73\left(\mathrm{~m}_{\mathrm{c}}, 2 \mathrm{H}\right), 7.86(\mathrm{~d}, J=15.7 \mathrm{~Hz}, 1 \mathrm{H}), 8.12\left(\mathrm{~m}_{\mathrm{c}}, 2 \mathrm{H}\right) .{ }^{13} \mathrm{C}$ NMR $\left(126 \mathrm{MHz}, \mathrm{CDCl}_{3}\right): \delta$ $122.2,127.5,128.4,128.6,129.1,129.3,130.7,135.1,137.1,140.1,144.9,145.7,190.1$. Elemental analysis was not performed.

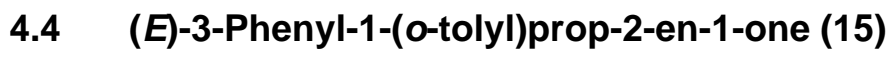

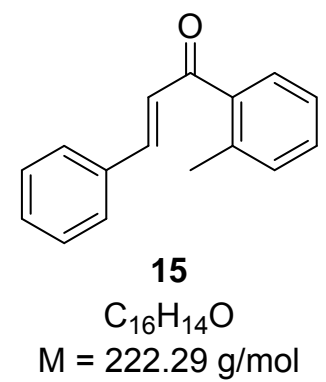

Prepared according to GP4 from benzaldehyde ( $0.45 \mathrm{~g}, 0.43 \mathrm{~mL}, 4.2 \mathrm{mmol}, 1.0$ equiv) and 1-(otolyl)ethan-1-one ( $0.56 \mathrm{~g}, 0.55 \mathrm{~mL}, 4.2 \mathrm{mmol}, 1.0$ equiv) in $90 \%$ yield $(0.84 \mathrm{~g}, 3.8 \mathrm{mmol})$ and with $E: Z=96: 4$. The product was obtained as a yellow oil. $\boldsymbol{R}_{\mathrm{f}}=0.41$ (cyclohexane/ethyl acetate 10/1). GLC (HP-5): $t_{R}=20.7 \mathrm{~min}$. IR (ATR): $\tilde{v}=3063(\mathrm{w}), 3027(\mathrm{w}), 1665(\mathrm{~m}), 1639(\mathrm{~m}), 1598(\mathrm{~s}), 1574$ (m), $1448(\mathrm{~m}), 1328(\mathrm{~m}), 1299(\mathrm{~m}), 1271(\mathrm{~m}), 1203(\mathrm{~m}), 978(\mathrm{~m}), 748(\mathrm{~s}), 695(\mathrm{~m}) \mathrm{cm}^{-1}$. HRMS (ESI) calculated for $\mathrm{C}_{16} \mathrm{H}_{13} \mathrm{O}[\mathrm{M}-\mathrm{H}]^{+}: 221.0961$; found: $221.0972 .{ }^{1} \mathrm{H} \mathrm{NMR}\left(500 \mathrm{MHz}, \mathrm{CDCl}_{3}\right): \delta 2.45$ (s, 3H), $7.14(\mathrm{~d}, J=16.0 \mathrm{~Hz}, 1 \mathrm{H}), 7.26-7.29(\mathrm{~m}, 2 \mathrm{H}), 7.37-7.42(\mathrm{~m}, 4 \mathrm{H}), 7.47(\mathrm{~d}, J=16.1 \mathrm{~Hz}, 1 \mathrm{H})$, 7.49-7.51 (m, 1H), 7.56-7.58 (m, 2H). ${ }^{13} \mathrm{C}$ NMR (126 MHz, $\left.\mathrm{CDCl}_{3}\right): \delta 20.3,125.6,126.9,128.2$, $128.6,129.1,130.6,130.8,131.5,134.8,137.1,139.2,146.0,196.7$. Elemental analysis was not performed. 


\section{5 (E)-1-(2-Methoxyphenyl)-3-phenylprop-2-en-1-one (16)}

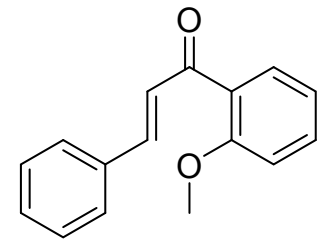

16

$\mathrm{C}_{16} \mathrm{H}_{14} \mathrm{O}_{2}$

$\mathrm{M}=238.29 \mathrm{~g} / \mathrm{mol}$

Prepared according to GP4 from benzaldehyde $(0.45 \mathrm{~g}, 0.43 \mathrm{~mL}, 4.2 \mathrm{mmol}, 1.0$ equiv) and 1-(2methoxyphenyl)ethan-1-one ( $0.63 \mathrm{~g}, 0.58 \mathrm{~mL}, 4.2 \mathrm{mmol}, 1.0$ equiv) in $87 \%$ yield $(0.87 \mathrm{~g}, 3.7 \mathrm{mmol})$ and with $E: Z>98: 2$. The product was obtained as a yellow oil after flash column chromatography on silica gel using cyclohexane/ethyl acetate (20/1) as eluent. $\boldsymbol{R}_{\mathrm{f}}=0.28$ (cyclohexane/ethyl acetate 10/1). GLC (HP-5): $t_{R}=22.2 \mathrm{~min}$. IR (ATR): $\tilde{v}=2839(\mathrm{w}), 1655(\mathrm{~m}), 1598(\mathrm{~s}), 1483(\mathrm{~m}), 1434(\mathrm{~m})$, $1330(\mathrm{~m}), 1239(\mathrm{~s}), 1202(\mathrm{~m}), 1111(\mathrm{~m}), 1015(\mathrm{~m}), 975(\mathrm{~m}), 868(\mathrm{w}), 808(\mathrm{w}), 752(\mathrm{~s}), 696(\mathrm{~m}) \mathrm{cm}^{-1}$. HRMS (ESI) calculated for $\mathrm{C}_{16} \mathrm{H}_{14} \mathrm{O}_{2}[\mathrm{M}]^{+}:$: 238.0988; found: $238.0988 .{ }^{1} \mathrm{H}$ NMR $\left(500 \mathrm{MHz}, \mathrm{CDCl}_{3}\right)$ : $\delta 3.90(\mathrm{~s}, 3 \mathrm{H}), 7.00-7.06(\mathrm{~m}, 2 \mathrm{H}), 7.36-7.40(\mathrm{~m}, 4 \mathrm{H}), 7.48\left(\mathrm{~m}_{\mathrm{c}}, 1 \mathrm{H}\right), 7.58-7.64(\mathrm{~m}, 4 \mathrm{H}) .{ }^{13} \mathrm{C}$ NMR $\left(126 \mathrm{MHz}, \mathrm{CDCl}_{3}\right): \delta 55.9,111.8,120.9,127.2,128.5,129.0,129.5,130.4,130.5,133.0,135.3$, $143.4,158.3,193.1$. Elemental analysis was not performed.

\section{6 (E)-1-Mesityl-3-phenylprop-2-en-1-one (17)}

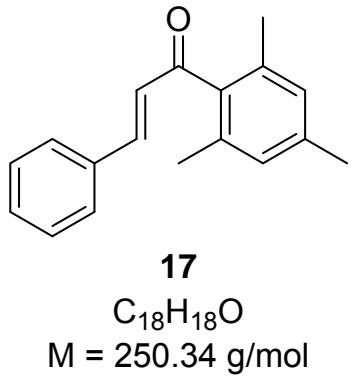

Prepared according to GP4 from benzaldehyde $(0.45 \mathrm{~g}, 0.43 \mathrm{~mL}, 4.2 \mathrm{mmol}, 1.0$ equiv) and 1mesitylethan-1-one ( $0.68 \mathrm{~g}, 0.70 \mathrm{~mL}, 4.2 \mathrm{mmol}, 1.0$ equiv) in $91 \%$ yield $(0.96 \mathrm{~g}, 3.8 \mathrm{mmol})$ and with $E: Z>98: 2$. The product was obtained as a yellow oil. $\boldsymbol{R}_{\mathbf{f}}=0.38$ (cyclohexane/ethyl acetate 10/1). GLC (HP-5): $t_{R}=21.9$ min. IR (ATR): $\tilde{v}=2922(w), 1640(\mathrm{~s}), 1598(\mathrm{~m}), 1448(\mathrm{~m}), 1378(\mathrm{w}), 1327$ (m), $1268(\mathrm{~s}), 1202(\mathrm{~m}), 1165(\mathrm{~m}), 1062(\mathrm{~s}), 1012(\mathrm{w}), 978(\mathrm{~m}), 851(\mathrm{w}), 768(\mathrm{~s}), 707(\mathrm{~m}), 683(\mathrm{~s})$ 
$\mathrm{cm}^{-1}$. HRMS (ESI) calculated for $\mathrm{C}_{18} \mathrm{H}_{19} \mathrm{O}[\mathrm{M}+\mathrm{H}]^{+}:$251.1430; found: $251.1428 .{ }^{1} \mathrm{H}$ NMR $(500 \mathrm{MHz}$, $\mathrm{CDCl}_{3}$ ): $\delta 2.20(\mathrm{~s}, 6 \mathrm{H}), 2.34(\mathrm{~s}, 3 \mathrm{H}), 6.90(\mathrm{~s}, 2 \mathrm{H}), 6.94(\mathrm{~d}, J=16.2 \mathrm{~Hz}, 1 \mathrm{H}), 7.20(\mathrm{~d}, J=16.3 \mathrm{~Hz}$, 1H), 7.36-7.41 (m, 3H), 7.50-7.52 (m, 2H). ${ }^{13} \mathrm{C}$ NMR (126 MHz, $\left.\mathrm{CDCl}_{3}\right): \delta 19.5,21.2,128.5,128.6$, $129.1,130.9,134.2,134.6,137.3,138.5,146.7,201.5$. Elemental analysis was not performed.

\section{7 (E)-1-(Naphthalen-1-yl)-3-phenylprop-2-en-1-one (18)}

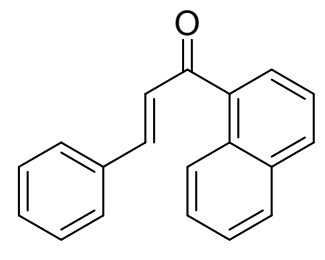

18

$\mathrm{C}_{19} \mathrm{H}_{14} \mathrm{O}$

$\mathrm{M}=258.32 \mathrm{~g} / \mathrm{mol}$

Prepared according to GP4 from benzaldehyde $(0.45 \mathrm{~g}, 0.43 \mathrm{~mL}, 4.2 \mathrm{mmol}, 1.0$ equiv) and 1(naphthalen-1-yl)ethan-1-one $(0.71 \mathrm{~g}, 0.64 \mathrm{~mL}, 4.2 \mathrm{mmol}, 1.0$ equiv) in $36 \%$ yield $(0.39 \mathrm{~g}$, $1.5 \mathrm{mmol}$ ) and with $E: Z=94: 6$. The product was obtained as a highly-viscous yellow oil after flash column chromatography on silica gel using cyclohexane/ethyl acetate $(10 / 1)$ as eluent. $\boldsymbol{R}_{\mathrm{f}}=0.36$ (cyclohexane/ethyl acetate 10/1). GLC (HP-5): $t_{R}=25.8 \mathrm{~min}$. IR (ATR): $\tilde{v}=3040(\mathrm{w}), 1952(\mathrm{w})$, $1637(\mathrm{~m}), 1595(\mathrm{~m}), 1508(\mathrm{~m}), 1448(\mathrm{~m}), 1283(\mathrm{~m}), 1098(\mathrm{~m}), 969(\mathrm{w}), 864(\mathrm{w}), 803(\mathrm{~m}), 811(\mathrm{~s})$, $776(\mathrm{~s}), 683(\mathrm{~m}) \mathrm{cm}^{-1}$. HRMS (ESI) calculated for $\mathrm{C}_{19} \mathrm{H}_{14} \mathrm{O}[\mathrm{M}]^{+*}: 258.1039$; found: 258.1050. ${ }^{1} \mathrm{H}$ NMR $\left(500 \mathrm{MHz}, \mathrm{CDCl}_{3}\right): \delta 7.29-7.33(\mathrm{~m}, 1 \mathrm{H}), 7.40-7.41(\mathrm{~m}, 3 \mathrm{H}), 7.53-7.63(\mathrm{~m}, 6 \mathrm{H}), 7.78(\mathrm{~d}, J=$ $7.0 \mathrm{~Hz}, 1 \mathrm{H}), 7.91-7.93(\mathrm{~m}, 1 \mathrm{H}), 8.00(\mathrm{~d}, J=8.3 \mathrm{~Hz}, 1 \mathrm{H}), 8.33(\mathrm{~d}, J=7.5 \mathrm{~Hz}, 1 \mathrm{H}) .{ }^{13} \mathrm{C}$ NMR $(126$ $\mathrm{MHz}_{\mathrm{CDCl}}$ ): $\delta 124.7,125.8,126.6,127.3,127.3,127.6,128.6,128.6,129.1,130.7,130.8,131.7$, $134.0,134.8,137.2,146.1,195.9$. Elemental analysis was not performed. 


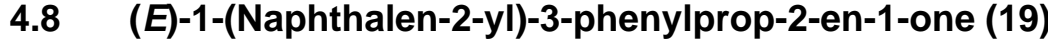

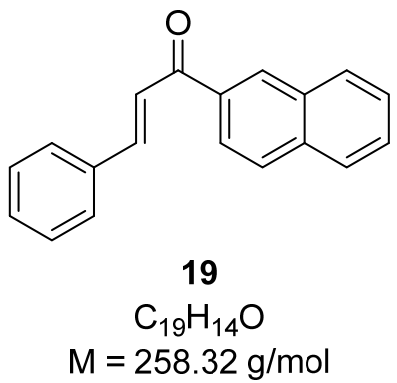

Prepared according to GP4 from benzaldehyde ( $0.45 \mathrm{~g}, 0.43 \mathrm{~mL}, 4.2 \mathrm{mmol}, 1.0$ equiv) and 1(naphthalen-2-yl)ethan-1-one $(0.71 \mathrm{~g}, 4.2 \mathrm{mmol}, 1.0$ equiv) in $91 \%$ yield $(0.98 \mathrm{~g}, 3.8 \mathrm{mmol}$ ) and with $E: Z>98: 2$. The product was obtained as a white solid. $\boldsymbol{R}_{\mathrm{f}}=0.34$ (cyclohexane/ethyl acetate 10/1). m.p.: $104{ }^{\circ} \mathrm{C}$ (pentane). GLC (HP-5): $t_{R}=26.8 \mathrm{~min}$. IR (ATR): $\tilde{v}=3055$ (w), $1660(\mathrm{~m}), 1601(\mathrm{~m})$, $1570(\mathrm{~m}), 1446(\mathrm{~m}), 1357(\mathrm{w}), 1283(\mathrm{w}), 1174(\mathrm{~m}), 1128(\mathrm{w}), 1049(\mathrm{w}), 983(\mathrm{~m}), 860(\mathrm{~m}), 819(\mathrm{~m})$, 758 (s), 685 (s) cm ${ }^{-1}$. HRMS (ESI) calculated for $\mathrm{C}_{19} \mathrm{H}_{13} \mathrm{O}[\mathrm{M}-\mathrm{H}]^{+}:$257.0961; found: 257.0950. ${ }^{1} \mathbf{H}$ NMR $\left(500 \mathrm{MHz}, \mathrm{CDCl}_{3}\right)$ : $\delta 7.43-7.47(\mathrm{~m}, 3 \mathrm{H}), 7.56-7.64(\mathrm{~m}, 2 \mathrm{H}), 7.69-7.72(\mathrm{~m}, 3 \mathrm{H}), 7.87-8.02(\mathrm{~m}$, $4 \mathrm{H}), 8.11(\mathrm{dd}, J=1.8 \mathrm{~Hz}, J=8.6 \mathrm{~Hz}, 1 \mathrm{H}), 8.55\left(\mathrm{~m}_{\mathrm{c}}, 1 \mathrm{H}\right) .{ }^{13} \mathrm{C} \mathrm{NMR}\left(126 \mathrm{MHz}, \mathrm{CDCl}_{3}\right): \delta 122.3$, 124.7, 126.9, 128.0, 128.5, 128.7, 128.7, 129.1, 129.7, 130.1, 130.7, 132.7, 135.2, 135.7, 135.7, $135.7,144.9,190.4$. Elemental analysis was not performed.

\section{$4.9 \quad(E)-1,3-D i($ naphthalen-2-yl)prop-2-en-1-one (20)}<smiles>O=C(/C=C/c1ccc2ccccc2c1)c1ccc2ccccc2c1</smiles>

Prepared according to GP4 from 2-naphthaldehyde (0.66 g, $4.2 \mathrm{mmol}, 1.0$ equiv) and 1(naphthalen-2-yl)ethan-1-one $(0.71 \mathrm{~g}, 4.2 \mathrm{mmol}, 1.00$ equiv) in $61 \%$ yield $(0.78 \mathrm{~g}, 2.5 \mathrm{mmol}$ ) and with $E: Z>98: 2$. The product was obtained as a yellow solid after flash column chromatography on silica gel using cyclohexane/ethyl acetate $(5 / 1) \rightarrow$ ethyl acetate as eluent. $\boldsymbol{R}_{\mathrm{f}}=0.31$ (cyclohexane/ethyl acetate 10/1). m.p.: $204{ }^{\circ} \mathrm{C}$ (ethyl acetate). GLC (HP-5): $t_{\mathrm{R}}=42.3 \mathrm{~min}$. IR (ATR): $\tilde{v}=3075(w), 1660(m), 1586(m), 1561(m), 1459(w), 1358(m), 1295(m), 1170(m), 1124$ 
(m), $1049(\mathrm{~m}), 984(\mathrm{~m}), 858(\mathrm{~m}), 814(\mathrm{~s}), 737(\mathrm{~s}) \mathrm{cm}^{-1}$. HRMS (ESI) calculated for $\mathrm{C}_{23} \mathrm{H}_{15} \mathrm{O}[\mathrm{M}-\mathrm{H}]^{+}$: 307.1117; found: 307.1106. ${ }^{1} \mathrm{H}$ NMR $\left(500 \mathrm{MHz}, \mathrm{CDCl}_{3}\right): \delta 7.52-7.64(\mathrm{~m}, 4 \mathrm{H}), 7.82(\mathrm{~d}, J=15.7 \mathrm{~Hz}$, $1 \mathrm{H}), 7.76-7.93(\mathrm{~m}, 5 \mathrm{H}), 7.97(\mathrm{~d}, J=8.7 \mathrm{~Hz}, 1 \mathrm{H}), 8.02-8.08(\mathrm{~m}, 3 \mathrm{H}), 8.14(\mathrm{dd}, J=1.7 \mathrm{~Hz}, J=$ $8.6 \mathrm{~Hz}, 1 \mathrm{H}), 8.59(\mathrm{~s}, 1 \mathrm{H}) .{ }^{13} \mathrm{C}$ NMR $\left(126 \mathrm{MHz} \mathrm{CDCl}_{3}\right): \delta 122.4,123.9,124.7,127.5,128.0,128.0$, $128.5,128.8,128.8,128.9,129.7,130.1,130.8,132.6,132.8,133.6,134.6,135.7,135.8,145.0$, 190.4 .

\section{General Procedure for Diels-Alder Reactions Catalyzed by Sulfur-Stabilized Silicon Cations (GP5)}

In a glovebox, a flame-dried Schlenk tube equipped with a magnetic stir bar is charged with $\left[\mathrm{Ph}_{3} \mathrm{C}\right]^{+}\left[\mathrm{B}\left(\mathrm{C}_{6} \mathrm{~F}_{5}\right)_{4}\right]^{-}(5.00 \mathrm{~mol} \%)$. The Schlenk tube is transferred to a fume hood and connected to a Schlenk line. After addition of $1,2-\mathrm{Cl}_{2} \mathrm{C}_{6} \mathrm{H}_{4}(0.1 \mathrm{~mL})$, a solution of the indicated hydrosilane $(5.50 \mathrm{~mol} \%)$ in $1,2-\mathrm{Cl}_{2} \mathrm{C}_{6} \mathrm{H}_{4}(0.2 \mathrm{~mL})$ is added, and the resulting mixture is stirred for $30 \mathrm{~min}$ at room temperature. Then, a solution of cyclohexa-1,3-diene (1, 2.10 equiv) and the indicated dienophile (1.00 equiv) in $1,2-\mathrm{Cl}_{2} \mathrm{C}_{6} \mathrm{H}_{4}(0.2 \mathrm{~mL})$ is added dropwise. The reaction mixture is stirred at room temperature for $3 \mathrm{~h}$, followed by the addition of saturated aqueous $\mathrm{NaHCO}_{3}$ solution $(2 \mathrm{~mL}$ ). The phases are separated, and the aqueous phase is extracted with tert-butyl methyl ether $(3 \times$ $10 \mathrm{~mL})$. The combined organic phases are washed with brine $(5 \mathrm{~mL})$, dried over $\mathrm{Na}_{2} \mathrm{SO}_{4}$, and concentrated under reduced pressure. The residue is purified by flash column chromatography on silica gel using cyclohexane/ethyl acetate mixtures as eluent to afford the Diels-Alder adduct. 


\section{1 endo-1-(-3-Phenylbicyclo[2.2.2]oct-5-en-2-yl)ethan-1-one (3)}

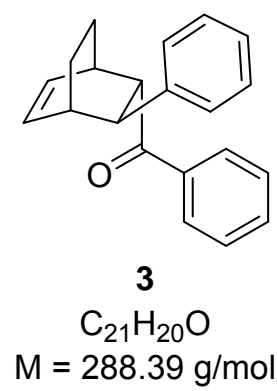

Diels-Alder reaction catalyzed by silicon cation (S,RS)-5:

Prepared according to GP5 from chalcone $(2,51.8 \mathrm{mg}, 0.249 \mathrm{mmol}, 1.00$ equiv) and cyclohexa-1,3diene (1, $50.0 \mu \mathrm{L}, 42.0 \mathrm{mg}, 0.523 \mathrm{mmol}, 2.1$ equiv) in $53 \%$ yield $(38.0 \mathrm{mg}, 0.132 \mathrm{mmol})$ and with endo:exo $=97: 3, \mathrm{dr}>95: 5$, and $25 \%$ ee. HPLC (Daicel Chiralcel OD-H, $20{ }^{\circ} \mathrm{C}, n$-heptane $/ i-\mathrm{PrOH}$ 97/3, flow rate: $0.65 \mathrm{~mL} / \mathrm{min}, \lambda=254 \mathrm{~nm}$ ): $t_{R}=10.8 \mathrm{~min}$ (major-3), $t_{\mathrm{R}}=12.6 \mathrm{~min}$ (minor-3).

Diels-Alder reaction catalyzed by silicon cation (S,S)-5:

Prepared according to GP5 from chalcone $(2,51.8 \mathrm{mg}, 0.249 \mathrm{mmol}, 1.00$ equiv) and cyclohexa-1,3diene (1, $50.0 \mu \mathrm{L}, 42.0 \mathrm{mg}, 0.523 \mathrm{mmol}, 2.1$ equiv) in $61 \%$ yield $(44.0 \mathrm{mg}, 0.152 \mathrm{mmol})$ and with endo:exo $=97: 3$, dr > 95:5, and $34 \%$ ee. HPLC (Daicel Chiralcel OD-H, $20{ }^{\circ} \mathrm{C}, n$-heptane $/ i-\mathrm{PrOH}$ 97/3, flow rate: $0.65 \mathrm{~mL} / \mathrm{min}, \lambda=254 \mathrm{~nm}$ ): $t_{\mathrm{R}}=11.0 \mathrm{~min}$ (major-3), $t_{\mathrm{R}}=12.8 \mathrm{~min}$ (minor-3).

Diels-Alder reaction catalyzed by silicon cation $(S, R)-5$ :

Prepared according to GP5 from chalcone $(2,51.8 \mathrm{mg}, 0.249 \mathrm{mmol}, 1.00$ equiv) and cyclohexa-1,3diene (1, $50.0 \mu \mathrm{L}, 42.0 \mathrm{mg}, 0.523 \mathrm{mmol}, 2.1$ equiv) in $57 \%$ yield $(41.0 \mathrm{mg}, 0.142 \mathrm{mmol})$ and with endo:exo $=97: 3, \mathrm{dr}>95: 5$, and $13 \%$ ee. HPLC (Daicel Chiralcel OD-H, $20{ }^{\circ} \mathrm{C}, n$-heptane $/ i$-PrOH 97/3, flow rate: $0.65 \mathrm{~mL} / \mathrm{min}, \lambda=254 \mathrm{~nm}$ ): $t_{\mathrm{R}}=10.8 \mathrm{~min}$ (major-3), $t_{\mathrm{R}}=12.6 \mathrm{~min}$ (minor-3).

The cycloadduct $\mathbf{3}$ was obtained as a white solid after flash column chromatography on silica gel using cyclohexane/ethyl acetate (20/1) as eluent. $\boldsymbol{R}_{\mathrm{f}}=0.33$ (cyclohexane/ethyl acetate 20/1). m.p.: $120-122{ }^{\circ} \mathrm{C}$ (cyclohexane) GLC (HP-5): $t_{\mathrm{R}}=24.1 \mathrm{~min}$ (exo-3), $t_{\mathrm{R}}=24.4 \mathrm{~min}$ (endo-3). HRMS (ESI) calculated for $\mathrm{C}_{21} \mathrm{H}_{21} \mathrm{O}[\mathrm{M}+\mathrm{H}]^{+}:$289.1587; found: $289.1582 .{ }^{1} \mathrm{H}$ NMR $\left(500 \mathrm{MHz}, \mathrm{CDCl}_{3}\right): \delta 1.10-1.18$ $(\mathrm{m}, 1 \mathrm{H}), 1.50\left(\mathrm{~m}_{\mathrm{c}}, 1 \mathrm{H}\right), 1.81-1.88(\mathrm{~m}, 1 \mathrm{H}), 1.88-1.96(\mathrm{~m}, 1 \mathrm{H}), 2.66-2.71(\mathrm{~m}, 1 \mathrm{H}), 2.97-3.01(\mathrm{~m}$, $1 \mathrm{H}), 3.48-3.52(\mathrm{~m}, 1 \mathrm{H}), 3.82(\mathrm{dd}, J=6.5 \mathrm{~Hz}, J=1.3 \mathrm{~Hz}, 1 \mathrm{H}), 6.13\left(\mathrm{~m}_{\mathrm{c}}, 1 \mathrm{H}\right), 6.58\left(\mathrm{~m}_{\mathrm{c}}, 1 \mathrm{H}\right), 7.20-$ $7.25(\mathrm{~m}, 1 \mathrm{H}), 7.29-7.36(\mathrm{~m}, 4 \mathrm{H}), 7.38-7.43(\mathrm{~m}, 2 \mathrm{H}), 7.49-7.54(\mathrm{~m}, 1 \mathrm{H}), 7.86-7.91\left(\mathrm{~m}_{\mathrm{c}}, 2 \mathrm{H}\right) .{ }^{13} \mathrm{C}$ NMR $\left(126 \mathrm{MHz}, \mathrm{CDCl}_{3}\right): \delta 18.6,26.6,34.7,36.6,44.9,51.1,126.3,128.2,128.5,128.6,130.8$, 
132.8, 136.4, 136.6, 143.0, 200.9. Elemental analysis was not performed. The analytical and spectroscopic data are in accordance with those reported. ${ }^{[33]}$

\section{2 endo-3-Phenylbicyclo[2.2.2]oct-5-en-2-yl)(p-tolyl)methanone (21)}

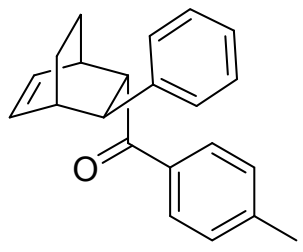

21

$\mathrm{C}_{22} \mathrm{H}_{22} \mathrm{O}$

$\mathrm{M}=302.42 \mathrm{~g} / \mathrm{mol}$

Prepared according to GP5 from (E)-3-phenyl-1-(p-tolyl)prop-2-en-1-one (12, $55.0 \mathrm{mg}, 0.249 \mathrm{mmol}$, 1.00 equiv) and cyclohexa-1,3-diene (1, $50.0 \mu \mathrm{L}, 42.0 \mathrm{mg}, 0.523 \mathrm{mmol}, 2.1$ equiv) in $67 \%$ yield $(51.0 \mathrm{mg}, 0.169 \mathrm{mmol}$ ) and with endo:exo $=96: 4, \mathrm{dr}>95: 5$, and $40 \%$ ee. The cycloadduct 21 was obtained as a white solid after flash column chromatography on silica gel using cyclohexane/ethyl acetate (50/1) as eluent. $\boldsymbol{R}_{\mathbf{f}}=0.62$ (cyclohexane/ethyl acetate 10/1). m.p.: $122{ }^{\circ} \mathrm{C}$ (pentane). GLC (HP-5): $t_{\mathrm{R}}=25.3 \mathrm{~min}$ (exo-21), $t_{\mathrm{R}}=25.7 \mathrm{~min}$ (endo-21). IR (ATR): $\tilde{\mathrm{v}}=2927$ (w), 2880 (w), 1671 (s), $1606(\mathrm{~m}), 1450(\mathrm{w}), 1230(\mathrm{~m}), 960(\mathrm{~m}), 818(\mathrm{~m}), 750(\mathrm{~m}), 700(\mathrm{~s}), 679(\mathrm{~m}) \mathrm{cm}^{-1}$. HRMS (ESI) calculated for $\mathrm{C}_{22} \mathrm{H}_{23} \mathrm{O}[\mathrm{M}+\mathrm{H}]^{+}: 303.1743$; found: $303.1743 .{ }^{1} \mathrm{H}$ NMR $\left(500 \mathrm{MHz}, \mathrm{CDCl}_{3}\right): \delta 1.09-1.15$ $(\mathrm{m}, 1 \mathrm{H}), 1.48\left(\mathrm{~m}_{\mathrm{c}}, 1 \mathrm{H}\right), 1.79-1.92(\mathrm{~m}, 2 \mathrm{H}), 2.38(\mathrm{~s}, 3 \mathrm{H}), 2.67-2.68(\mathrm{~m}, 1 \mathrm{H}), 2.95-2.97(\mathrm{~m}, 1 \mathrm{H})$, $3.47-3.49(\mathrm{~m}, 1 \mathrm{H}), 3.78(\mathrm{dd}, J=6.6 \mathrm{~Hz}, J=1.6 \mathrm{~Hz}, 1 \mathrm{H}), 6.11\left(\mathrm{~m}_{\mathrm{c}}, 1 \mathrm{H}\right), 6.54-6.57(\mathrm{~m}, 1 \mathrm{H}), 7.18-$ $7.22(\mathrm{~m}, 3 \mathrm{H}), 7.28-7.33(\mathrm{~m}, 4 \mathrm{H}), 7.78(\mathrm{mc}, 2 \mathrm{H}) .{ }^{13} \mathrm{C} \mathrm{NMR}\left(126 \mathrm{MHz}, \mathrm{CDCl}_{3}\right): \delta 18.7,21.7,26.6$, 34.8, 36.6, 44.9, 51.0, 126.3, 128.3, 128.6, 128.7, 129.3, 130.9, 134.0, 136.3, 143.1, 143.6, 200.5. HPLC (Daicel Chiralcel OD-H, $20{ }^{\circ} \mathrm{C}, n$-heptane/i-PrOH $=97: 3$, flow rate: $0.65 \mathrm{~mL} / \mathrm{min}, \lambda=254$ $\mathrm{nm}): t_{\mathrm{R}}=10.2 \mathrm{~min}\left(\right.$ major-21), $t_{\mathrm{R}}=12.1 \mathrm{~min}($ minor-21). Elemental analysis results are outside the tolerance range. 
5.3 endo-(4-(tert-Butyl)phenyl)(-3-phenylbicyclo[2.2.2]oct-5-en-2-yl)methanone (22)

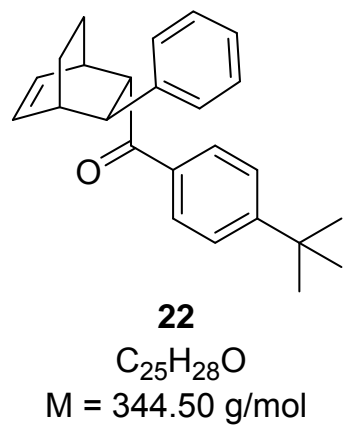

Prepared according to GP5 from (E)-1-(4-(tert-butyl)phenyl)-3-phenylprop-2-en-1-one (13, $66.0 \mathrm{mg}$, $0.249 \mathrm{mmol}, 1.00$ equiv) and cyclohexa-1,3-diene (1, $50.0 \mu \mathrm{L}, 42.0 \mathrm{mg}, 0.523 \mathrm{mmol}, 2.1$ equiv) in $72 \%$ yield $(62.0 \mathrm{mg}, 0.180 \mathrm{mmol})$ and with endo:exo $=97: 3, \mathrm{dr}>95: 5$, and $53 \%$ ee. The cycloadduct 22 was obtained as a white solid after flash column chromatography on silica gel using cyclohexane/ethyl acetate $(50 / 1)$ as eluent. $\boldsymbol{R}_{\mathbf{f}}=0.69$ (cyclohexane/ethyl acetate 10/1). m.p.: $122{ }^{\circ} \mathrm{C}$ (pentane) GLC (HP-5): $t_{\mathrm{R}}=27.7 \mathrm{~min}\left(\right.$ exo-22), $t_{\mathrm{R}}=28.1 \mathrm{~min}($ endo-22). IR (ATR): $\tilde{\mathrm{v}}=2941$ $(\mathrm{w}), 2870(\mathrm{w}), 1671(\mathrm{~s}), 1605(\mathrm{~m}), 1408(\mathrm{w}), 1229(\mathrm{~m}), 1109(\mathrm{~m}), 963(\mathrm{~m}), 825(\mathrm{~m}), 811(\mathrm{~s}), 745$ (m), $694(\mathrm{~s}) \mathrm{cm}^{-1}$. HRMS (ESI) calculated for $\mathrm{C}_{25} \mathrm{H}_{29} \mathrm{O}[\mathrm{M}+\mathrm{H}]^{+}: 345.2213$; found: $345.2214 .{ }^{1} \mathbf{H}$ NMR $\left(500 \mathrm{MHz}, \mathrm{CDCl}_{3}\right): \delta 1.10-1.17(\mathrm{~m}, 1 \mathrm{H}), 1.33(\mathrm{~s}, 9 \mathrm{H}), 1.48\left(\mathrm{~m}_{\mathrm{c}}, 1 \mathrm{H}\right), 1.81-1.93(\mathrm{~m}, 2 \mathrm{H}), 2.69-2.71$ (m, 1H), 2.97-2.98 (m, 1H), 3.52-3.54 (m, 1H), $3.81(\mathrm{dd}, J=6.5 \mathrm{~Hz}, J=1.5 \mathrm{~Hz}, 1 \mathrm{H}), 6.10\left(\mathrm{~m}_{\mathrm{c}}, 1 \mathrm{H}\right)$, $6.57\left(m_{c}, 1 H\right), 7.18-7.22(m, 1 H), 7.28-7.33(m, 4 H), 7.43\left(m_{c}, 2 H\right), 7.85\left(m_{c}, 2 H\right) .{ }^{13} \mathrm{C}$ NMR $(126$ $\left.\mathrm{MHz}, \mathrm{CDCl}_{3}\right): \delta 18.6,26.7,31.2,34.9,35.2,36.5,44.7,50.9,125.6,126.2,128.3,128.5,128.5$, $130.8,133.9,136.4,143.1,156.5,200.4$. Anal. Calcd for $\mathrm{C}_{25} \mathrm{H}_{28} \mathrm{O}$ : C, 87.16; $\mathrm{H}, 8.19$. Found: C, 87.24; H, 8.38. HPLC (Daicel Chiralcel OD-H, $20{ }^{\circ} \mathrm{C}, n$-heptane/i-PrOH 97/3, flow rate: 0.65 $\mathrm{mL} / \mathrm{min}, \lambda=254 \mathrm{~nm}$ ): $t_{\mathrm{R}}=12.2 \mathrm{~min}$ (major-22), $t_{\mathrm{R}}=14.1 \mathrm{~min}$ (minor-22). 


\section{4 endo-[1,1'-Biphenyl]-4-yl(-3-phenylbicyclo[2.2.2]oct-5-en-2-yl)methanone (23)}

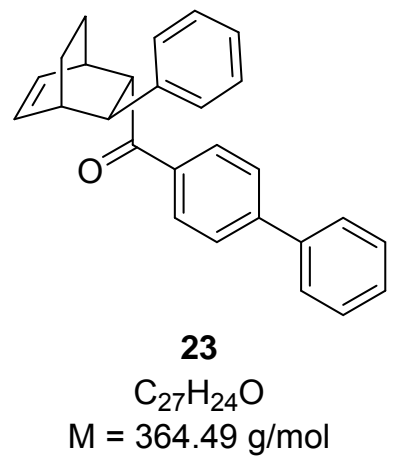

Prepared according to GP5 from (E)-1-([1,1'-biphenyl]-4-yl)-3-phenylprop-2-en-1-one (14, 71.0 mg, $0.249 \mathrm{mmol}, 1.00$ equiv) and cyclohexa-1,3-diene (1, $50.0 \mu \mathrm{L}, 42.0 \mathrm{mg}, 0.523 \mathrm{mmol}, 2.1$ equiv) in $71 \%$ yield $(65.0 \mathrm{mg}, 0.178 \mathrm{mmol})$ and with endo:exo $=96: 4, \mathrm{dr}>95: 5$, and $55 \%$ ee. The cycloadduct $\mathbf{2 3}$ was obtained as a white solid after flash column chromatography on silica gel using cyclohexane/ethyl acetate $(50 / 1)$ as eluent. $\boldsymbol{R}_{\mathbf{f}}=0.59$ (cyclohexane/ethyl acetate 10/1). m.p.: $120^{\circ} \mathrm{C}$ (pentane). GLC (HP-5): $t_{\mathrm{R}}=38.5 \mathrm{~min}$ (exo-23), $t_{\mathrm{R}}=39.5 \mathrm{~min}$ (endo-23). IR (ATR): $\tilde{\mathrm{v}}=2950$ (w), $2937(w), 1670(\mathrm{~s}), 1602(\mathrm{~m}), 1404(\mathrm{~m}), 1228(\mathrm{~m}), 962(\mathrm{~m}), 829(\mathrm{w}), 743(\mathrm{~m}), 701$ (s), $692(\mathrm{~s})$ $\mathrm{cm}^{-1}$. HRMS (ESI) calculated for $\mathrm{C}_{27} \mathrm{H}_{24} \mathrm{O}[\mathrm{M}]^{+\bullet}: 364.1822$; found: 364.1820 . ${ }^{1} \mathrm{H}$ NMR $(500 \mathrm{MHz}$, $\left.\mathrm{CD}_{2} \mathrm{Cl}_{2}\right): \delta 1.01-1.07(\mathrm{~m}, 1 \mathrm{H}), 1.36-1.43(\mathrm{~m}, 1 \mathrm{H}), 1.70-1.76(\mathrm{~m}, 1 \mathrm{H}), 1.83-1.89(\mathrm{~m}, 1 \mathrm{H}), 2.57-2.59$ $(\mathrm{m}, 1 \mathrm{H}), 2.92-2.93(\mathrm{~m}, 1 \mathrm{H}), 3.38-3.39(\mathrm{~m}, 1 \mathrm{H}), 3.74-3.75(\mathrm{~m}, 1 \mathrm{H}), 6.02\left(\mathrm{~m}_{\mathrm{c}}, 1 \mathrm{H}\right), 6.47\left(\mathrm{~m}_{\mathrm{c}}, 1 \mathrm{H}\right)$, $7.12\left(m_{c}, 1 H\right), 7.22-7.26(m, 4 H), 7.29-7.32(m, 1 H), 7.38\left(m_{c}, 2 H\right), 7.53-7.57(m, 4 H), 7.85(d, J=$ $8.4 \mathrm{~Hz}, 2 \mathrm{H}) .{ }^{13} \mathrm{C}$ NMR $\left(126 \mathrm{MHz}, \mathrm{CDCl}_{3}\right): \delta 18.9,26.8,35.1,37.1,45.1,51.3,126.5,127.5,127.6$, 128.6, 128.8, 129.3, 129.4, 131.2, 135.7, 136.6, 140.3, 143.3, 145.6, 200.5. HPLC (Daicel Chiralcel OD-H, $20^{\circ} \mathrm{C}, n$-heptane/i-PrOH 97/3, flow rate: $0.65 \mathrm{~mL} / \mathrm{min}, \lambda=254 \mathrm{~nm}$ ): $t_{\mathrm{R}}=26.6 \mathrm{~min}($ minor-23), $t_{\mathrm{R}}=33.3 \mathrm{~min}$ (major-23). Elemental analysis results are outside the tolerance range. 


\section{5 endo-3-Phenylbicyclo[2.2.2]oct-5-en-2-yl)(o-tolyl)methanone (24)}

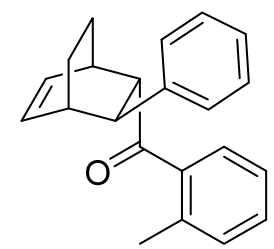

24

$$
\begin{gathered}
\mathrm{C}_{22} \mathrm{H}_{22} \mathrm{O} \\
\mathrm{M}=302.42 \mathrm{~g} / \mathrm{mol}
\end{gathered}
$$

Prepared according to GP5 from (E)-3-phenyl-1-(o-tolyl)prop-2-en-1-one (15, $55.0 \mathrm{mg}, 0.249 \mathrm{mmol}$, 1.00 equiv) and cyclohexa-1,3-diene (1, $50.0 \mu \mathrm{L}, 42.0 \mathrm{mg}, 0.523 \mathrm{mmol}, 2.1$ equiv) in $50 \%$ yield $(38.0 \mathrm{mg}, 0.126 \mathrm{mmol}$ ) and with endo:exo $=96: 4, \mathrm{dr}>95: 5$, and $31 \%$ ee. The cycloadduct 24 was obtained as a white solid after flash column chromatography on silica gel using cyclohexane/ethyl acetate (50/1) as eluent. $\boldsymbol{R}_{\mathbf{f}}=0.67$ (cyclohexane/ethyl acetate 10/1). m.p.: $114-116{ }^{\circ} \mathrm{C}$ (pentane). GLC (HP-5): $t_{R}=24.5 \mathrm{~min}\left(\right.$ exo-24), $t_{R}=24.6 \mathrm{~min}$ (endo-24). IR (ATR): $\tilde{\mathrm{v}}=2942(\mathrm{w}), 2873(\mathrm{w})$, 2168 (w), 1683 (s), 1450 (w), 1230 (w), 1036 (w), $961(\mathrm{~m}), 885$ (w), 783 (w), $746(\mathrm{~m}), 702(\mathrm{~s}), 683$ $(\mathrm{m}), 660(\mathrm{w}) \mathrm{cm}^{-1}$. HRMS (ESI) calculated for $\mathrm{C}_{22} \mathrm{H}_{23} \mathrm{O}[\mathrm{M}+\mathrm{H}]^{+}:$303.1743; found: 303.1745. ${ }^{1} \mathrm{H}$ NMR $\left(500 \mathrm{MHz}, \mathrm{CDCl}_{3}\right): \delta 1.05-1.09(\mathrm{~m}, 1 \mathrm{H}), 1.40-1.44(\mathrm{~m}, 1 \mathrm{H}), 1.77\left(\mathrm{~m}_{\mathrm{c}}, 2 \mathrm{H}\right), 2.35(\mathrm{~s}, 3 \mathrm{H}), 2.63-2.64$ $(\mathrm{m}, 1 \mathrm{H}), 2.89-2.90(\mathrm{~m}, 1 \mathrm{H}), 3.46\left(\mathrm{~m}_{\mathrm{c}}, 1 \mathrm{H}\right), 3.60(\mathrm{dd}, J=6.5 \mathrm{~Hz}, J=1.5 \mathrm{~Hz}, 1 \mathrm{H}), 6.12\left(\mathrm{~m}_{\mathrm{c}}, 1 \mathrm{H}\right), 6.57$ $\left(\mathrm{m}_{\mathrm{c}}, 1 \mathrm{H}\right), 7.13\left(\mathrm{~m}_{\mathrm{c}}, 1 \mathrm{H}\right), 7.20-7.23(\mathrm{~m}, 2 \mathrm{H}), 7.27-7.34(\mathrm{~m}, 6 \mathrm{H}) .{ }^{13} \mathrm{C}$ NMR $\left(126 \mathrm{MHz}, \mathrm{CDCl}_{3}\right): \delta 18.7$, 20.6, 26.5, 33.8, 36.9, 44.4, 54.4, 125.5, 126.3, 127.2, 128.3, 128.4, 128.6, 128.6, 130.4, 130.8, 131.5, 136.9, 137.3, 139.2, 143.0, 205.7. HPLC (Daicel Chiralcel OD-H, $20{ }^{\circ} \mathrm{C}, n$-heptane/i-PrOH 97/3, flow rate: $0.65 \mathrm{~mL} / \mathrm{min}, \lambda=210 \mathrm{~nm}$ ): $t_{R}=8.4 \mathrm{~min}$ (major-24), $t_{\mathrm{R}}=9.1 \mathrm{~min}$ (minor-24). Elemental analysis results are outside the tolerance range. 


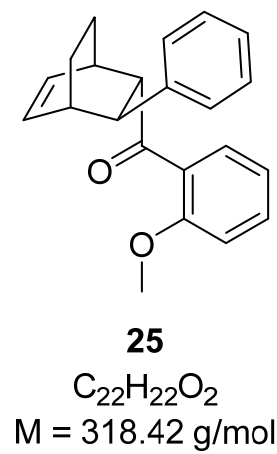

Prepared according to GP5 from (E)-1-(2-methoxyphenyl)-3-phenylprop-2-en-1-one (16, $59.3 \mathrm{mg}$, $0.249 \mathrm{mmol}, 1.00$ equiv) and cyclohexa-1,3-diene (1, $50.0 \mu \mathrm{L}, 42.0 \mathrm{mg}, 0.523 \mathrm{mmol}, 2.1$ equiv) in $69 \%$ yield $(54.5 \mathrm{mg}, 0.171 \mathrm{mmol})$ and with endo:exo $=97: 3, \mathrm{dr}>95: 5$, and $7 \%$ ee. The cycloadduct 25 was obtained as a white solid after flash column chromatography on silica gel using cyclohexane/ethyl acetate (50/1) as eluent. $\boldsymbol{R}_{\mathbf{f}}=0.42$ (cyclohexane/ethyl acetate 10/1). m.p.: $66{ }^{\circ} \mathrm{C}$ (cyclohexane). GLC (HP-5): $t_{\mathrm{R}}=25.6 \mathrm{~min}\left(\right.$ exo-25), $t_{\mathrm{R}}=25.8 \mathrm{~min}$ (endo-25). IR (ATR): $\tilde{\mathrm{v}}=3041$ (w), 2940 (m), 2869 (w), 1683 (s), 1594 (m), 1483 (m), 1395 (w), 1285 (s), 1244 (s), 1110 (m), 1015 (s), $960(\mathrm{~m}), 748$ (s), 682 (s) cm ${ }^{-1}$. HRMS (ESI) calculated for $\mathrm{C}_{22} \mathrm{H}_{22} \mathrm{O}_{2}$ [M] ${ }^{+}:$318.1614; found: 318.1623. ${ }^{1} \mathrm{H}$ NMR $\left(500 \mathrm{MHz}, \mathrm{CDCl}_{3}\right): \delta 1.02-1.08(\mathrm{~m}, 1 \mathrm{H}), 1.37-1.44(\mathrm{~m}, 1 \mathrm{H}), 1.70-1.79(\mathrm{~m}, 2 \mathrm{H})$, 2.59-2.60 (m, 1H), $2.95(\mathrm{~s}, 1 \mathrm{H}), 3.39-3.41(\mathrm{~m}, 1 \mathrm{H}), 3.69(\mathrm{~s}, 3 \mathrm{H}), 3.90(\mathrm{dd}, J=6.5 \mathrm{~Hz}, J=1.2 \mathrm{~Hz}$, $1 \mathrm{H}), 6.11\left(\mathrm{~m}_{\mathrm{c}}, 1 \mathrm{H}\right), 6.52\left(\mathrm{~m}_{\mathrm{c}}, 1 \mathrm{H}\right), 6.82(\mathrm{~d}, J=8.8 \mathrm{~Hz}, 1 \mathrm{H}), 6.94\left(\mathrm{~m}_{\mathrm{c}}, 1 \mathrm{H}\right), 7.16-7.19(\mathrm{~m}, 1 \mathrm{H}), 7.25-$ $7.31(\mathrm{~m}, 4 \mathrm{H}), 7.36\left(\mathrm{~m}_{\mathrm{c}}, 1 \mathrm{H}\right), 7.41(\mathrm{dd}, J=7.6 \mathrm{~Hz}, J=1.7 \mathrm{~Hz}, 1 \mathrm{H}) .{ }^{13} \mathrm{C}$ NMR $\left(126 \mathrm{MHz}, \mathrm{CDCl}_{3}\right): \delta$ 18.7, 26.5, 33.5, 37.0, 44.8, 55.0, 55.4, 111.3, 120.8, 126.0, 128.3, 128.3, 129.3, 130.1, 131.3, 132.4, 136.6, 143.4, 157.4, 204.5. HPLC (Daicel Chiralcel OD-H, $20{ }^{\circ} \mathrm{C}$, $n$-heptane/i-PrOH = 97:3, flow rate: $0.65 \mathrm{~mL} / \mathrm{min}, \lambda=210 \mathrm{~nm}$ ): $t_{\mathrm{R}}=17.3 \mathrm{~min}$ (major-25), $t_{\mathrm{R}}=21.0 \mathrm{~min}$ (minor-25). Elemental analysis was not performed. 


\section{7 endo-Naphthalen-1-yl(-3-phenylbicyclo[2.2.2]oct-5-en-2-yl)methanone (27)}

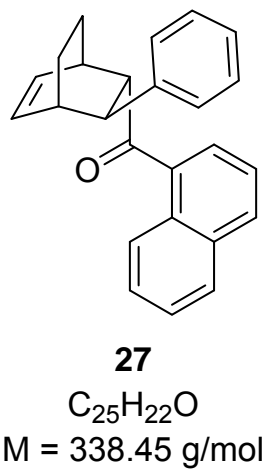

Prepared according to GP5 from (E)-1-(naphthalen-1-yl)-3-phenylprop-2-en-1-one (18, 64.0 mg, $0.249 \mathrm{mmol}, 1.00$ equiv) and cyclohexa-1,3-diene (1, $50.0 \mu \mathrm{L}, 42.0 \mathrm{mg}, 0.523 \mathrm{mmol}, 2.1$ equiv) in $68 \%$ yield $(57.0 \mathrm{mg}, 0.168 \mathrm{mmol})$ and with endo:exo $=95: 5, \mathrm{dr}>95: 5$, and $15 \%$ ee. The cycloadduct $\mathbf{2 7}$ was obtained as a white solid after flash column chromatography on silica gel using cyclohexane/ethyl acetate (50/1) as eluent. $\boldsymbol{R}_{\mathbf{f}}=0.59$ (cyclohexane/ethyl acetate 10/1). m.p.: $100-$ $102{ }^{\circ} \mathrm{C}$ (pentane). GLC (HP-5): $t_{R}=30.7 \mathrm{~min}$ (exo-27), $t_{\mathrm{R}}=30.9 \mathrm{~min}$ (endo-27). IR (ATR): $\tilde{\mathrm{v}}=2957$ (w), $2867(w), 1678(\mathrm{~m}), 1507(\mathrm{w}), 1231(\mathrm{~m}), 1103(\mathrm{~m}), 943(\mathrm{~m}), 779(\mathrm{~s}), 751(\mathrm{~m}), 702(\mathrm{~m}), 690(\mathrm{~s})$ $\mathrm{cm}^{-1}$. HRMS (ESI) calculated for $\mathrm{C}_{25} \mathrm{H}_{23} \mathrm{O}[\mathrm{M}+\mathrm{H}]^{+}: 339.1743$; found: $339.1745 .{ }^{1} \mathrm{H}$ NMR $(500 \mathrm{MHz}$, $\left.\mathrm{CDCl}_{3}\right): \delta 1.04-1.11(\mathrm{~m}, 1 \mathrm{H}), 1.38-1.45(\mathrm{~m}, 1 \mathrm{H}), 1.75-1.82(\mathrm{~m}, 2 \mathrm{H}), 2.65-2.66(\mathrm{~m}, 1 \mathrm{H}), 2.92-2.93$ $(\mathrm{m}, 1 \mathrm{H}), 3.56\left(\mathrm{~m}_{\mathrm{c}}, 1 \mathrm{H}\right), 3.77(\mathrm{dd}, J=6.4 \mathrm{~Hz}, J=1.5 \mathrm{~Hz}, 1 \mathrm{H}), 6.19\left(\mathrm{~m}_{\mathrm{c}}, 1 \mathrm{H}\right), 6.62\left(\mathrm{~m}_{\mathrm{c}}, 1 \mathrm{H}\right), 7.24\left(\mathrm{~m}_{\mathrm{c}}\right.$, 1H), 7.29-7.38 (m, 5H), 7.50-7.54 (m, 3H), 7.84-7.86 (m, 1H), $7.89(\mathrm{~d}, J=8.2 \mathrm{~Hz}, 1 \mathrm{H}), 8.10-8.13$ (m, 1H). ${ }^{13} \mathrm{C}$ NMR $\left(126 \mathrm{MHz}, \mathrm{CDCl}_{3}\right): \delta 18.8,26.4,33.8,37.0,44.4,55.3,124.4,125.5,125.6$, 126.3, 126.5, 127.5, 128.3, 128.5, 128.6, 130.6, 131.0, 131.3, 133.9, 136.9, 137.7, 143.0, 205.5. HPLC (Daicel Chiralcel OD-H, $20^{\circ} \mathrm{C}, n$-heptane/i-PrOH 97/3, flow rate: $0.65 \mathrm{~mL} / \mathrm{min}, \lambda=210 \mathrm{~nm}$ ): $t_{\mathrm{R}}=12.5 \mathrm{~min}$ (minor-27), $t_{\mathrm{R}}=14.2 \mathrm{~min}$ (major-27). Elemental analysis results are outside the tolerance range. 
5.8 endo-Naphthalen-2-yl(-3-phenylbicyclo[2.2.2]oct-5-en-2-yl)methanone (28)

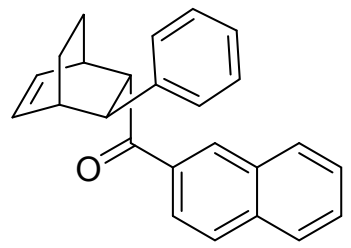

$$
\begin{gathered}
28 \\
\mathrm{C}_{25} \mathrm{H}_{22} \mathrm{O} \\
\mathrm{M}=338.45 \mathrm{~g} / \mathrm{mol}
\end{gathered}
$$

Prepared according to GP5 from (E)-1-(naphthalen-2-yl)-3-phenylprop-2-en-1-one (19, $64.0 \mathrm{mg}$, $0.249 \mathrm{mmol}, 1.00$ equiv) and cyclohexa-1,3-diene (1, $50.0 \mu \mathrm{L}, 42.0 \mathrm{mg}, 0.523 \mathrm{mmol}, 2.1$ equiv) in $61 \%$ yield $(51.0 \mathrm{mg}, 0.151 \mathrm{mmol})$ and with endo:exo $=96: 4, \mathrm{dr}>95: 5$, and $50 \%$ ee. The cycloadduct 28 was obtained as a white solid after flash column chromatography on silica gel using cyclohexane/ethyl acetate $(50 / 1)$ as eluent. $\boldsymbol{R}_{\mathbf{f}}=0.62$ (cyclohexane/ethyl acetate 10/1). m.p.: $160{ }^{\circ} \mathrm{C}$ (cyclohexane). GLC (HP-5): $t_{\mathrm{R}}=32.3 \mathrm{~min}\left(\right.$ exo-28), $t_{\mathrm{R}}=33.1 \mathrm{~min}$ (endo-28). IR (ATR): $\tilde{\mathrm{v}}=$ 2955 (w), 1674 (s), 1186 (w), 938 (w), 831 (m), 811 (s), 746 (s), 698 (s) cm cm $^{-1}$ HRMS (ESI) calculated for $\mathrm{C}_{25} \mathrm{H}_{23} \mathrm{O}[\mathrm{M}+\mathrm{H}]^{+}: 339.1743$; found: $339.1745 .{ }^{1} \mathrm{H}$ NMR $\left(500 \mathrm{MHz}, \mathrm{CDCl}_{3}\right): \delta 1.13-1.19$ $(\mathrm{m}, 1 \mathrm{H}), 1.50-1.56(\mathrm{~m}, 1 \mathrm{H}), 1.86-1.99(\mathrm{~m}, 2 \mathrm{H}), 2.68\left(\mathrm{~m}_{\mathrm{c}}, 1 \mathrm{H}\right), 3.06-3.07(\mathrm{~m}, 1 \mathrm{H}), 3.44\left(\mathrm{~m}_{\mathrm{c}}, 1 \mathrm{H}\right)$, $3.94(\mathrm{dd}, J=6.6 \mathrm{~Hz}, 1.6 \mathrm{~Hz}, 1 \mathrm{H}), 6.20-6.23\left(\mathrm{~m}_{\mathrm{c}}, 1 \mathrm{H}\right), 6.55-6.58\left(\mathrm{~m}_{\mathrm{c}}, 1 \mathrm{H}\right), 7.22-7.25(\mathrm{~m}, 1 \mathrm{H}), 7.32-$ $7.36(\mathrm{~m}, 4 \mathrm{H}), 7.48-7.51(\mathrm{~m}, 1 \mathrm{H}), 7.54-7.58(\mathrm{~m}, 1 \mathrm{H}), 7.74(\mathrm{~d}, J=8.2 \mathrm{~Hz}, 1 \mathrm{H}), 7.84(\mathrm{~d}, J=8.5 \mathrm{~Hz}$, $2 \mathrm{H}), 7.96$ (dd, $J=8.7 \mathrm{~Hz}, 1.8 \mathrm{~Hz}, 1 \mathrm{H}), 8.24(\mathrm{~s}, 1 \mathrm{H}) .{ }^{13} \mathrm{C} \mathrm{NMR}\left(126 \mathrm{MHz}, \mathrm{CDCl}_{3}\right): \delta 18.3,26.4,34.5$, 36.7, 45.6, 51.7, 124.7, 126.4, 126.7, 127.8, 128.4, 128.4 (3C), 128.7 (2C), 129.6, 130.2, 131.5, 132.6, 133.8, 135.5, 136.0, 143.2, 201.0. HPLC (Daicel Chiralcel OD-H, $20{ }^{\circ} \mathrm{C}, n$-heptane/i-PrOH 97/3, flow rate: $0.65 \mathrm{~mL} / \mathrm{min}, \lambda=254 \mathrm{~nm}$ ): $t_{\mathrm{R}}=16.6 \mathrm{~min}$ (major-28), $t_{\mathrm{R}}=21.6 \mathrm{~min}$ (minor-28). Elemental analysis results are outside the tolerance range. 
5.9 endo-Naphthalen-2-yl(-3-(naphthalen-2-yl)bicyclo[2.2.2]oct-5-en-2-yl)methanone (29)

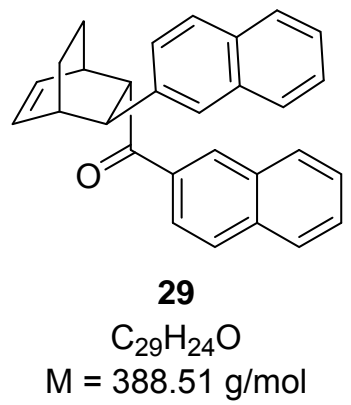

Prepared according to GP5 from (E)-1,3-di(naphthalen-2-yl)prop-2-en-1-one $(\mathbf{2 0}, 76 \mathrm{mg}$, $0.249 \mathrm{mmol}, 1.00$ equiv) and cyclohexa-1,3-diene (1, 50.0 $\mu \mathrm{L}, 42.0 \mathrm{mg}, 0.523 \mathrm{mmol}, 2.1$ equiv) in $58 \%$ yield (56 mg, $0.144 \mathrm{mmol}$ ) and with $59 \%$ ee. The cycloadduct 29 was obtained as a white solid after flash column chromatography on silica gel using cyclohexane/ethyl acetate (50/1) as eluent. $\boldsymbol{R}_{\mathbf{f}}=0.57$ (cyclohexane/ethyl acetate 10/1). m.p.: 150-154 ${ }^{\circ} \mathrm{C}$ (pentane). IR (ATR): $\tilde{v}=2940(\mathrm{w})$, 2869 (w), 1674 (s), 1460 (w), 1275 (w), 1123 (m), 937 (w), 853 (w), 811 (s), 734 (s), 693 (s) cm cm. $^{-1}$ HRMS (ESI) calculated for $\mathrm{C}_{29} \mathrm{H}_{24} \mathrm{O}[\mathrm{M}]^{+*}: 338.1822$; found: $338.1826 .{ }^{1} \mathbf{H}$ NMR $\left(500 \mathrm{MHz}, \mathrm{CD}_{2} \mathrm{Cl}_{2}\right)$ : ס 1.13-1.20 (m, 1H), 1.50-1.56 (m, 1H), 1.87-1.93 (m, 1H), 2.03-2.09 (m, 1H), 2.77-2.78 (m, 1H), 3.09-3.10 (m, 1H), 3.62-3.64 (m, 1H), $4.08(\mathrm{dd}, J=6.6 \mathrm{~Hz}, J=1.3 \mathrm{~Hz}, 1 \mathrm{H}), 6.17\left(\mathrm{~m}_{\mathrm{c}}, 1 \mathrm{H}\right), 6.59$ $\left(\mathrm{m}_{\mathrm{c}}, 1 \mathrm{H}\right), 7.42-7.48(\mathrm{~m}, 4 \mathrm{H}), 7.54-7.57(\mathrm{~m}, 1 \mathrm{H}), 7.74(\mathrm{~d}, J=8.4 \mathrm{~Hz}, 1 \mathrm{H}), 7.80-7.86(\mathrm{~m}, 6 \mathrm{H}), 7.96$ (dd, $J=1.7 \mathrm{~Hz}, J=8.6 \mathrm{~Hz}, 1 \mathrm{H}), 8.34(\mathrm{~s}, 1 \mathrm{H}) .{ }^{13} \mathrm{C}$ NMR $\left(126 \mathrm{MHz}, \mathrm{CD}_{2} \mathrm{Cl}_{2}\right): \delta 19.0,26.8,35.1,37.2$, $45.6,51.5,124.8,125.8,126.4,127.0,127.8,127.8,128.0,128.4,128.6,128.7,129.8,130.2$, 131.6, 132.6, 132.9, 134.0, 134.2, 135.8, 136.4, 140.9, 200.9. HPLC (Daicel Chiralcel OD-H, $20{ }^{\circ} \mathrm{C}$, $n$-heptane $/ i-\mathrm{PrOH}$ 97/3, flow rate: $0.65 \mathrm{~mL} / \mathrm{min}, \lambda=254 \mathrm{~nm}$ ): $t_{\mathrm{R}}=26.7 \mathrm{~min}$ (major-29), $t_{\mathrm{R}}=42.2 \mathrm{~min}$ (minor-29). Elemental analysis results are outside the tolerance range. 


\subsection{0 endo-Phenyl(-3-phenylbicyclo[2.2.2]oct-5-en-2-yl)methanone (31)}

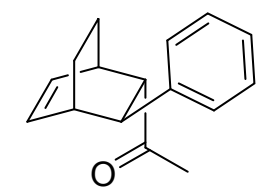

31

$\mathrm{C}_{16} \mathrm{H}_{18} \mathrm{O}$

$\mathrm{M}=226.32 \mathrm{~g} / \mathrm{mol}$

Prepared according to GP5 from (E)-4-phenylbut-3-en-2-one $(\mathbf{3 0}, 36.4, \mathrm{mg}, 0.249 \mathrm{mmol}$, 1.00 equiv) and cyclohexa-1,3-diene (1, $50.0 \mu \mathrm{L}, 42.0 \mathrm{mg}, 0.523 \mathrm{mmol}, 2.1$ equiv) in $63 \%$ yield (33.0 $\mathrm{mg}, 0.155 \mathrm{mmol}$ ) and with endo:exo $=98: 2, \mathrm{dr}>95: 5$, and $4 \%$ ee. The cycloadduct 31 was obtained as a sticky colorless oil after flash column chromatography on silica gel using cyclohexane/ethyl acetate (50/1) as eluent. $\boldsymbol{R}_{\mathbf{f}}=0.20$ (cyclohexane/ethyl acetate 20/1). GLC (HP$5): t_{\mathrm{R}}=18.2 \mathrm{~min}\left(\right.$ exo-31), $t_{\mathrm{R}}=18.7 \mathrm{~min}$ (endo-31). HRMS (ESI) calculated for $\mathrm{C}_{16} \mathrm{H}_{19} \mathrm{O}[\mathrm{M}+\mathrm{H}]^{+}$: 227.1430; found: 227.1429. ${ }^{1} \mathbf{H}$ NMR $\left(500 \mathrm{MHz}, \mathrm{CDCl}_{3}\right): \delta 1.00-1.08(\mathrm{~m}, 1 \mathrm{H}), 1.41-1.49(\mathrm{~m}, 1 \mathrm{H})$, 1.65-1.76 (m, 2H), $2.03(\mathrm{~s}, 3 \mathrm{H}), 2.51-2.55(\mathrm{~m}, 1 \mathrm{H}), 2.92-2.95(\mathrm{~m}, 1 \mathrm{H}), 3.00-3.04(\mathrm{~m}, 1 \mathrm{H}), 3.10-$ $3.14(\mathrm{~m}, 1 \mathrm{H}), 6.21\left(\mathrm{~m}_{\mathrm{c}}, 1 \mathrm{H}\right), 6.47\left(\mathrm{~m}_{\mathrm{c}}, 1 \mathrm{H}\right), 7.23\left(\mathrm{~m}_{\mathrm{c}}, 1 \mathrm{H}\right), 7.27-7.31(\mathrm{~m}, 2 \mathrm{H}), 7.32-7.38(\mathrm{~m}, 2 \mathrm{H})$. ${ }^{13} \mathrm{C}$ NMR $\left(126 \mathrm{MHz}, \mathrm{CDCl}_{3}\right): \delta 18.5,26.1,28.4,32.7,37.3,45.6,56.6,126.5,128.2,128.6,131.6$, 136.1, 142.8, 208.9. HPLC (Daicel Chiralcel OD-H, $20{ }^{\circ} \mathrm{C}$, $n$-heptane/i-PrOH 98/2, flow rate: $0.65 \mathrm{~mL} / \mathrm{min}, \lambda=210 \mathrm{~nm}$ ): $t_{\mathrm{R}}=14.1 \mathrm{~min}$ (major-31), $t_{\mathrm{R}}=15.2 \mathrm{~min}$ (minor-31). Elemental analysis was not performed. The analytical and spectroscopic data are in accordance with those reported. ${ }^{\text {[S3] }}$ 


\section{$6 \quad$ NMR spectra}

Figure S1. ${ }^{1} \mathrm{H}$ NMR (500 MHz, $\left.\mathrm{CDCl}_{3}\right)$ of rac-9.
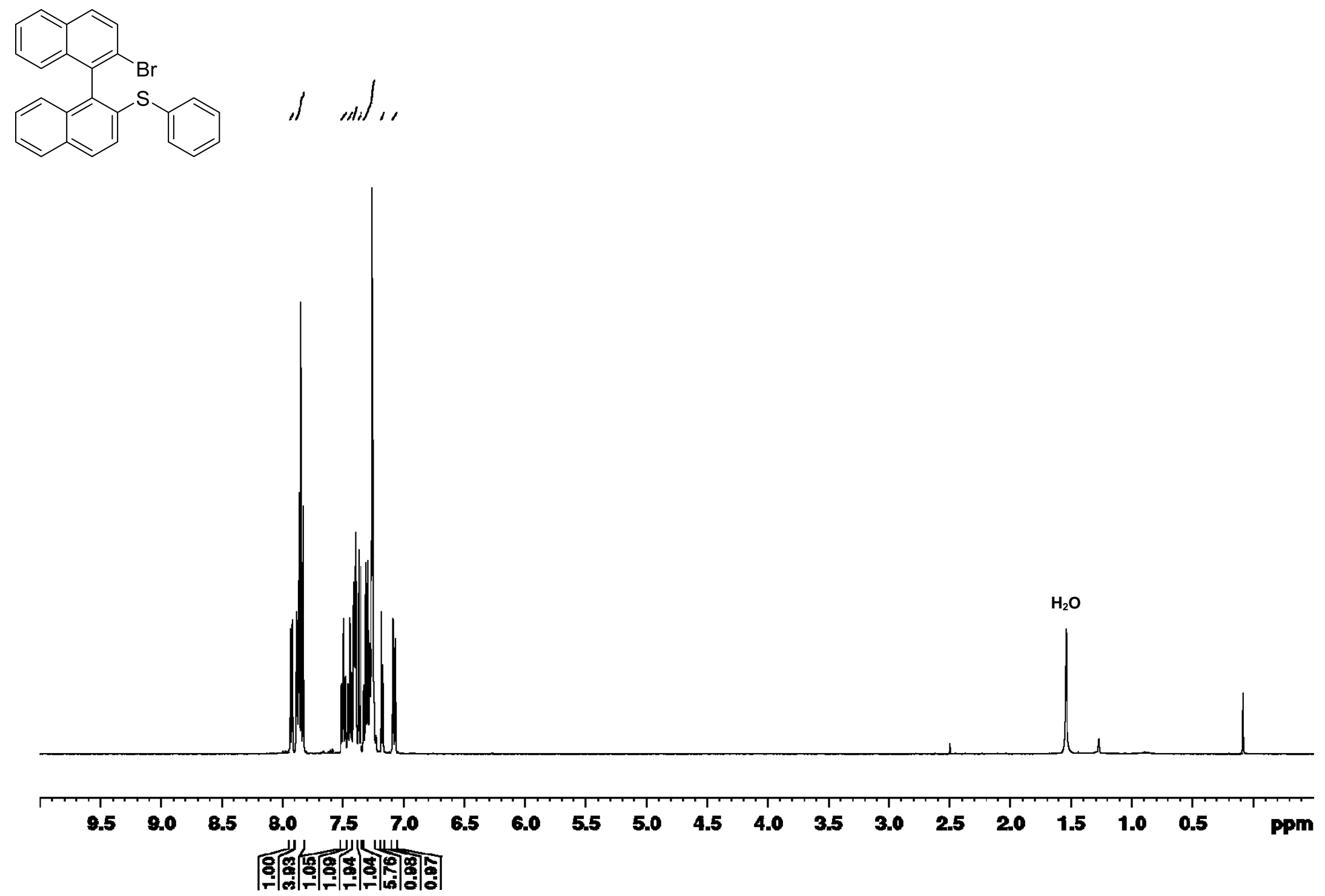
Supporting Information for Organometallics

S32

Figure S2. ${ }^{13} \mathrm{C}$ NMR (126 MHz, $\left.\mathrm{CDCl}_{3}\right)$ of rac-9.

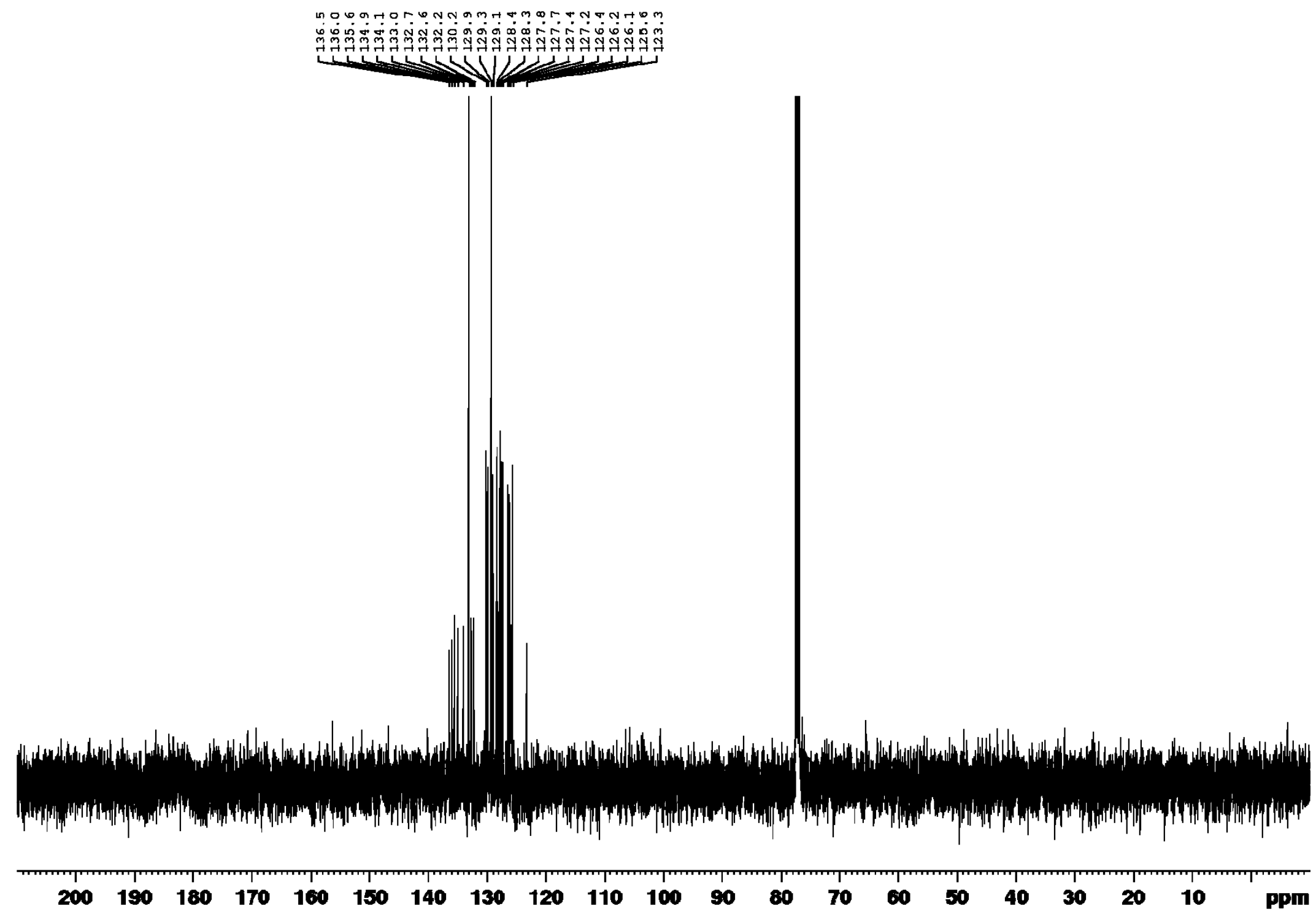


Figure S3. ${ }^{1} \mathrm{H} \mathrm{NMR}\left(500 \mathrm{MHz}, \mathrm{CDCl}_{3}\right)$ of $(S, R S)-11$.

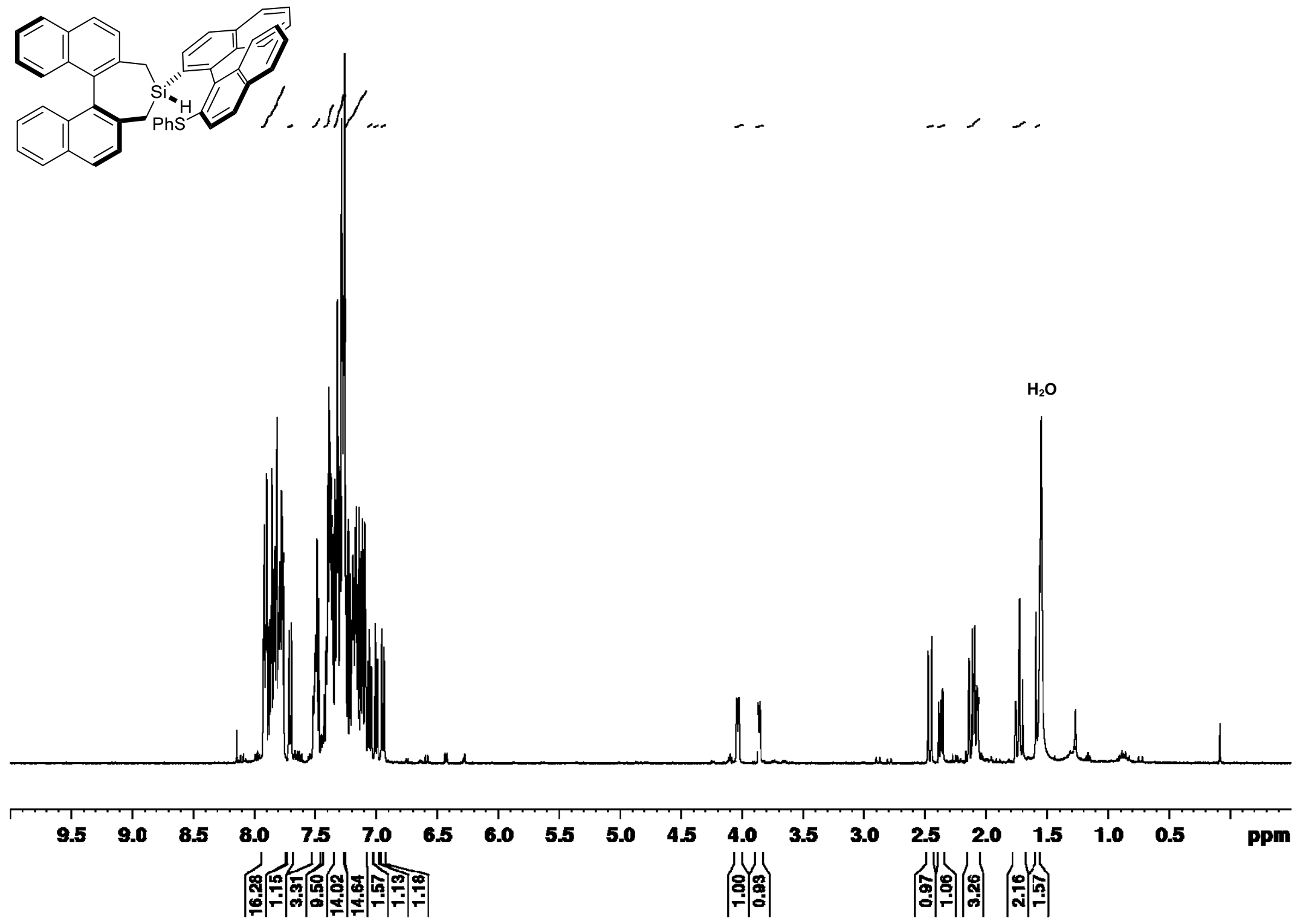


Figure S4. ${ }^{13} \mathrm{C} \mathrm{NMR}\left(126 \mathrm{MHz}, \mathrm{CDCl}_{3}\right)$ of $(S, R S)-\mathbf{1 1}$.

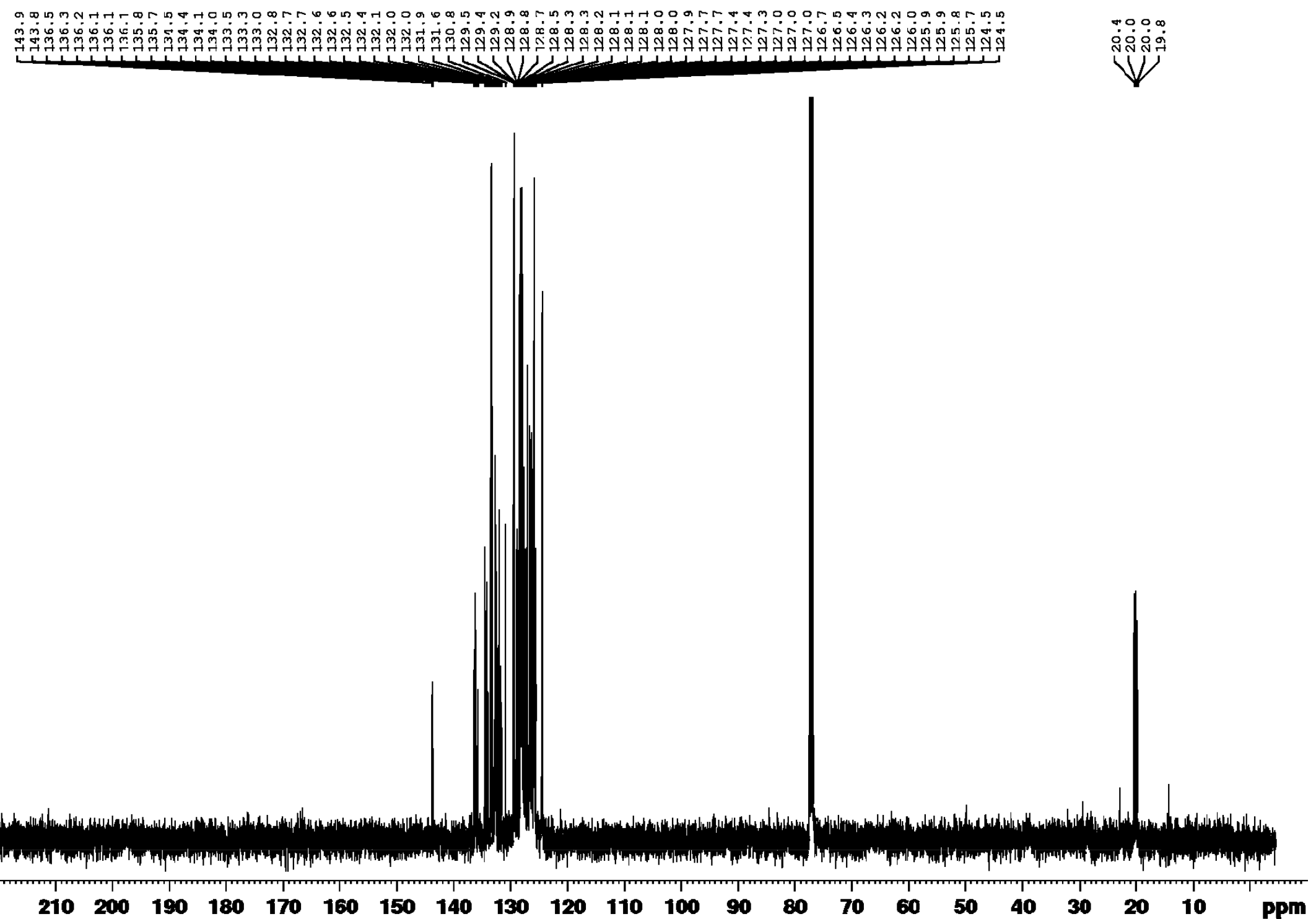


Figure S5. ${ }^{29} \mathrm{Si}$ DEPT $\left(99 \mathrm{MHz}, \mathrm{CDCl}_{3}\right)$ of $(S, R S)-11$.

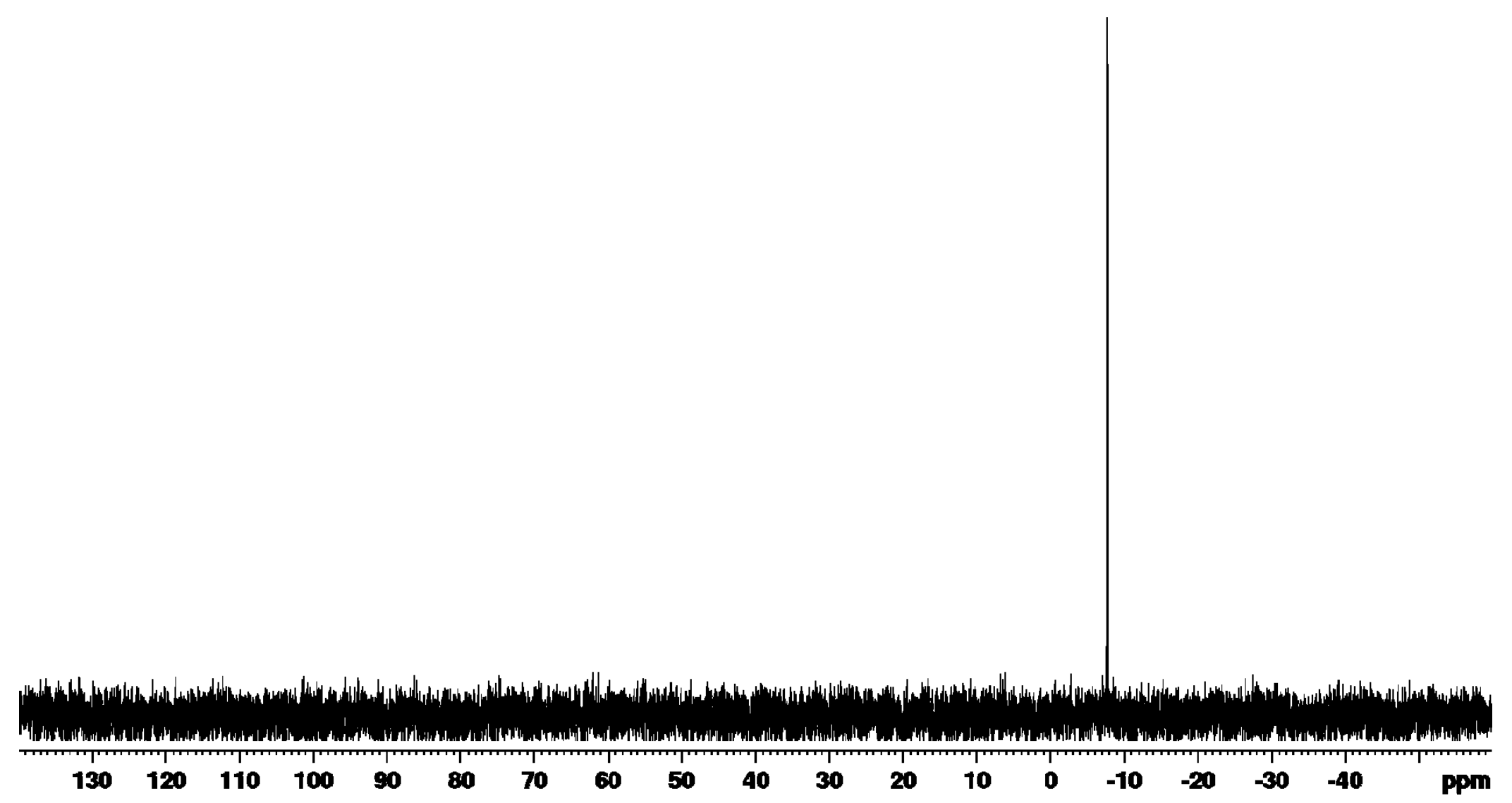


Figure S6. ${ }^{1} \mathrm{H} \mathrm{NMR}\left(500 \mathrm{MHz}, \mathrm{CDCl}_{3}\right)$ of $(\mathrm{S}, \mathrm{S})-\mathbf{1 1}$.

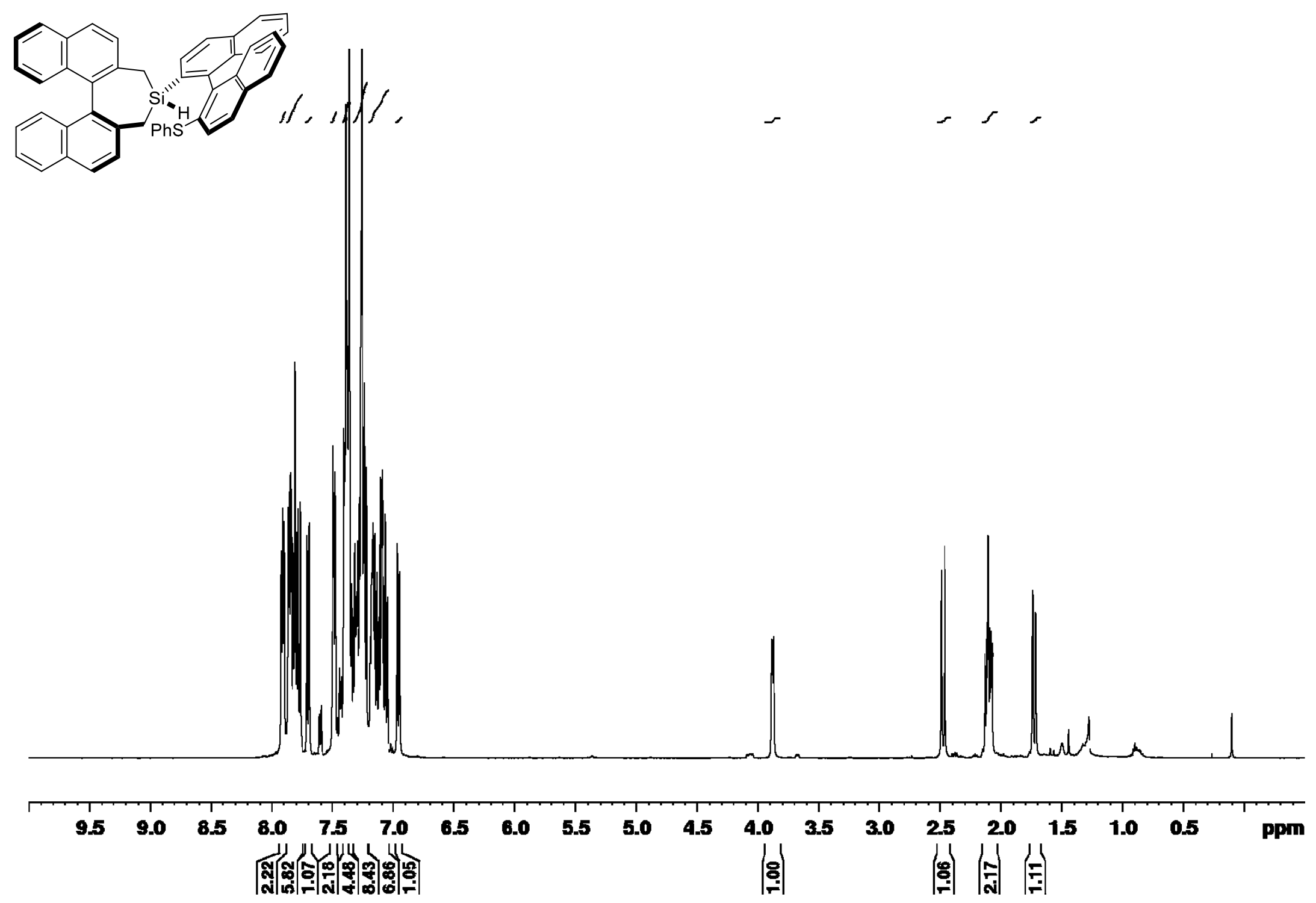


Figure S7. ${ }^{13} \mathrm{C} \mathrm{NMR}\left(126 \mathrm{MHz}, \mathrm{CDCl}_{3}\right)$ of $(S, S)-11$.

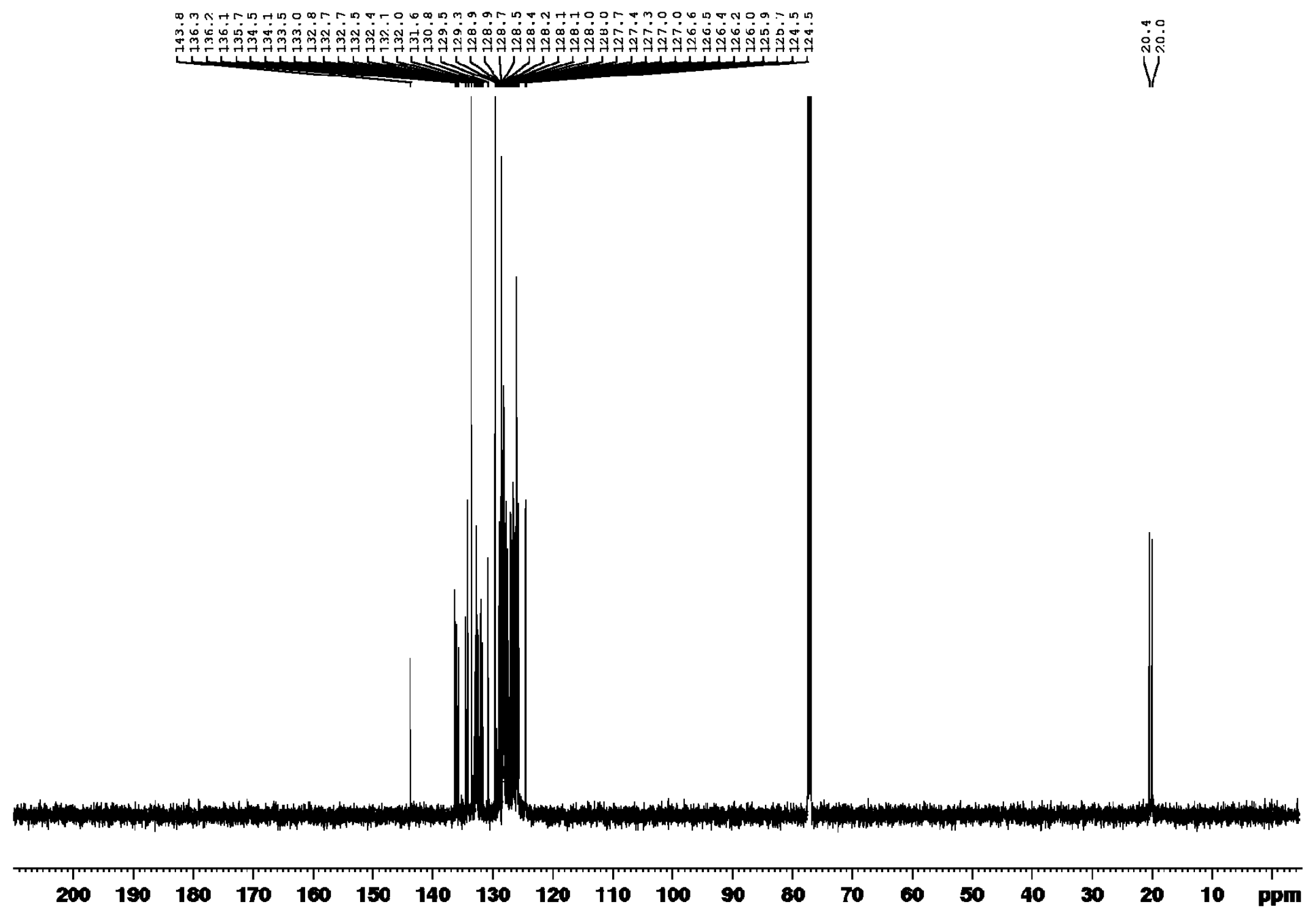


Figure S8. ${ }^{29} \mathrm{Si}$ DEPT $\left(99 \mathrm{MHz}, \mathrm{CDCl}_{3}\right)$ of $(\mathrm{S}, S)$-11.

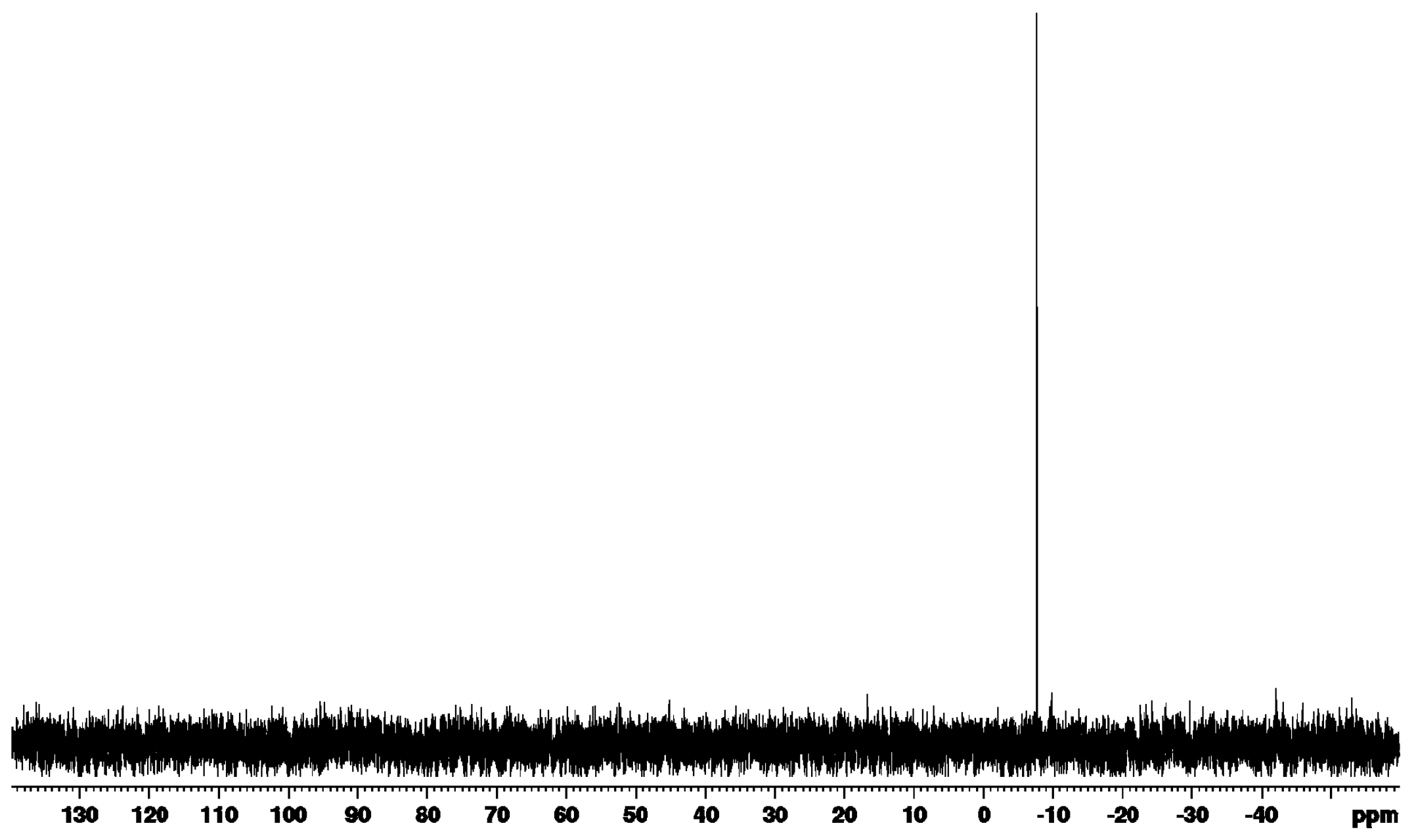


Figure S9. ${ }^{1} \mathrm{H} \mathrm{NMR}\left(500 \mathrm{MHz}, \mathrm{CDCl}_{3}\right)$ of $(S, R)-11$.

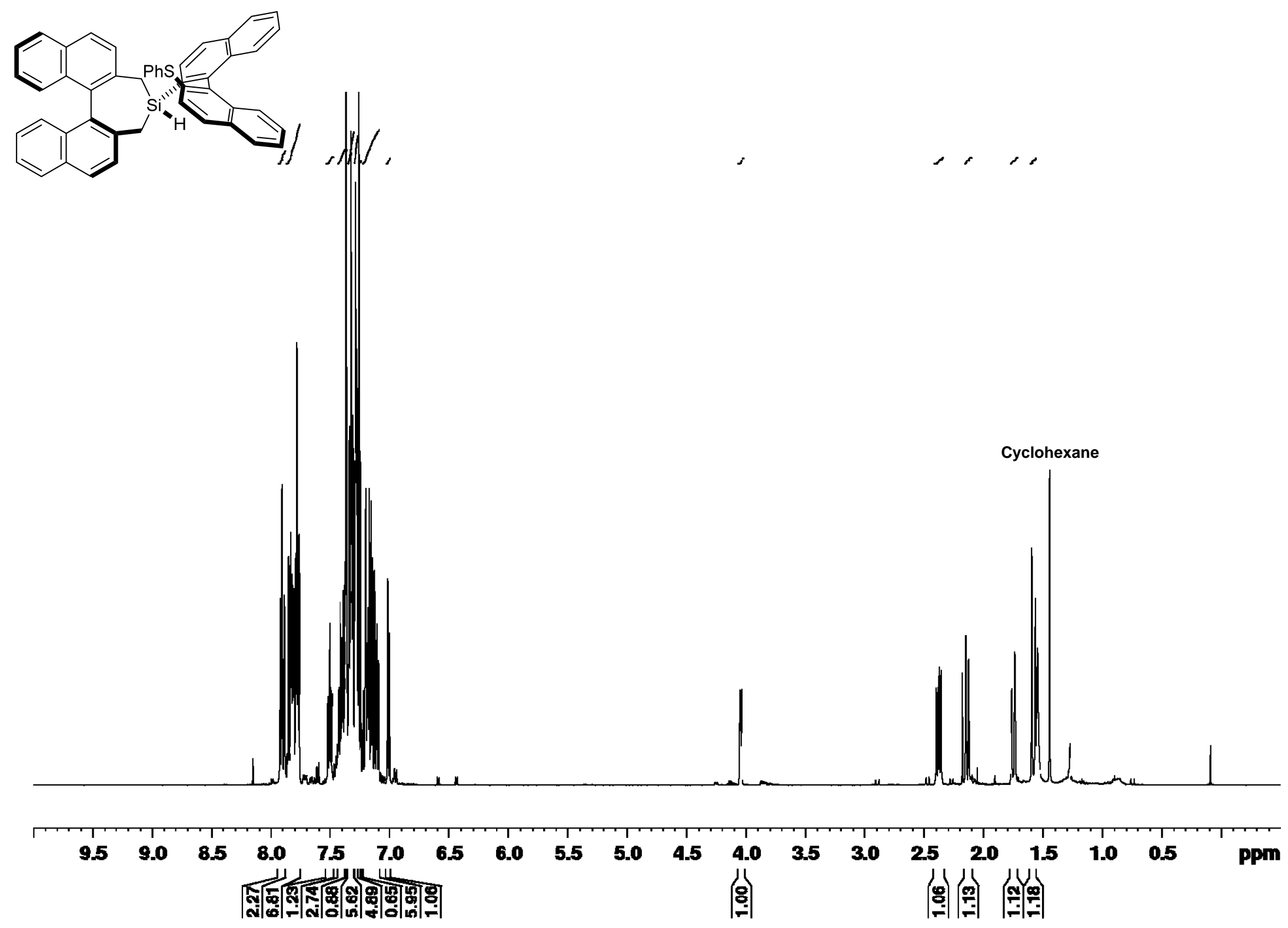


Figure S10. ${ }^{13} \mathrm{C}$ NMR $\left(126 \mathrm{MHz}, \mathrm{CDCl}_{3}\right)$ of $(S, R)-11$.

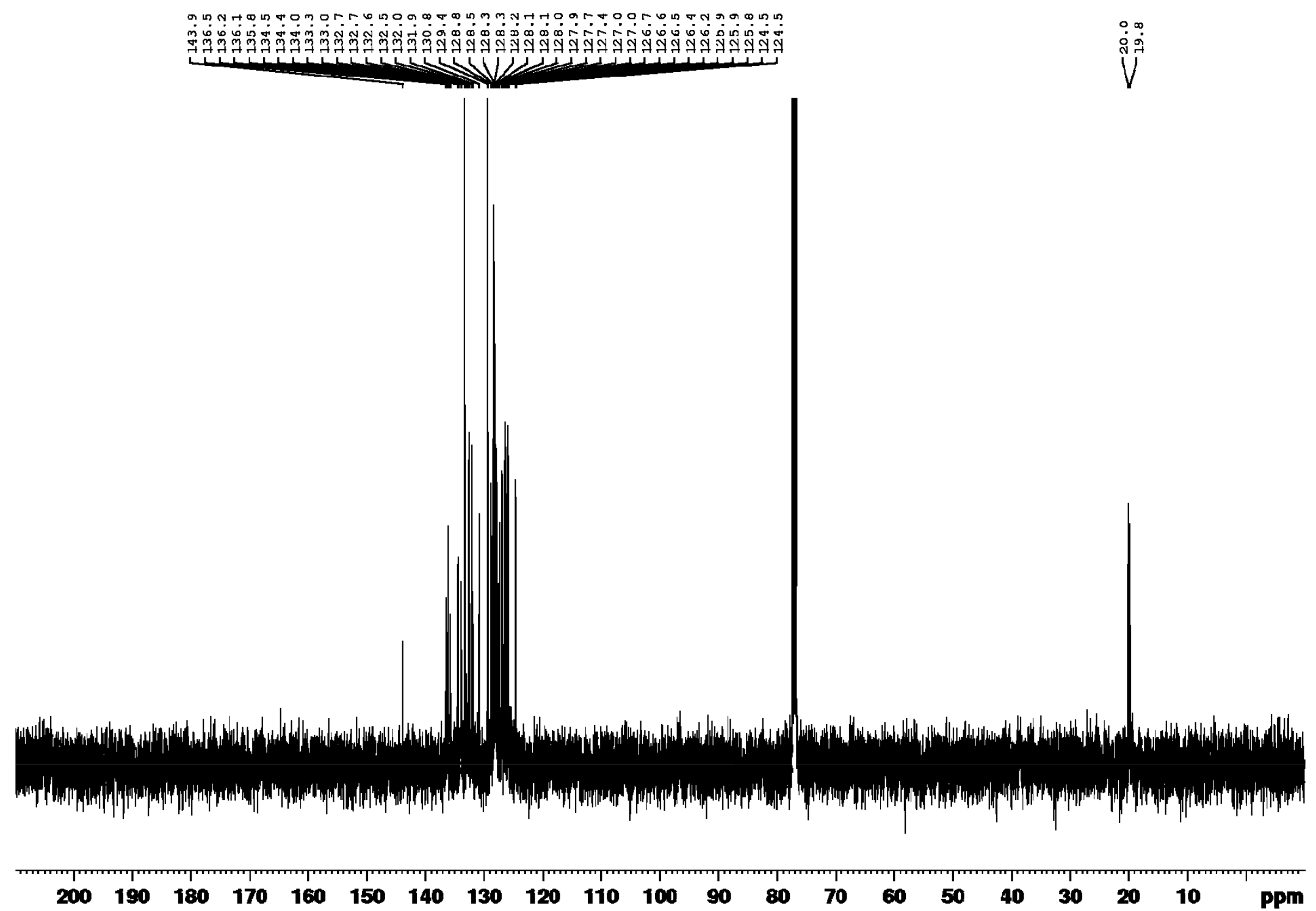


Figure S11. ${ }^{29} \mathrm{Si}$ DEPT $\left(99 \mathrm{MHz}, \mathrm{CDCl}_{3}\right)$ of $(S, R)-\mathbf{1 1}$.

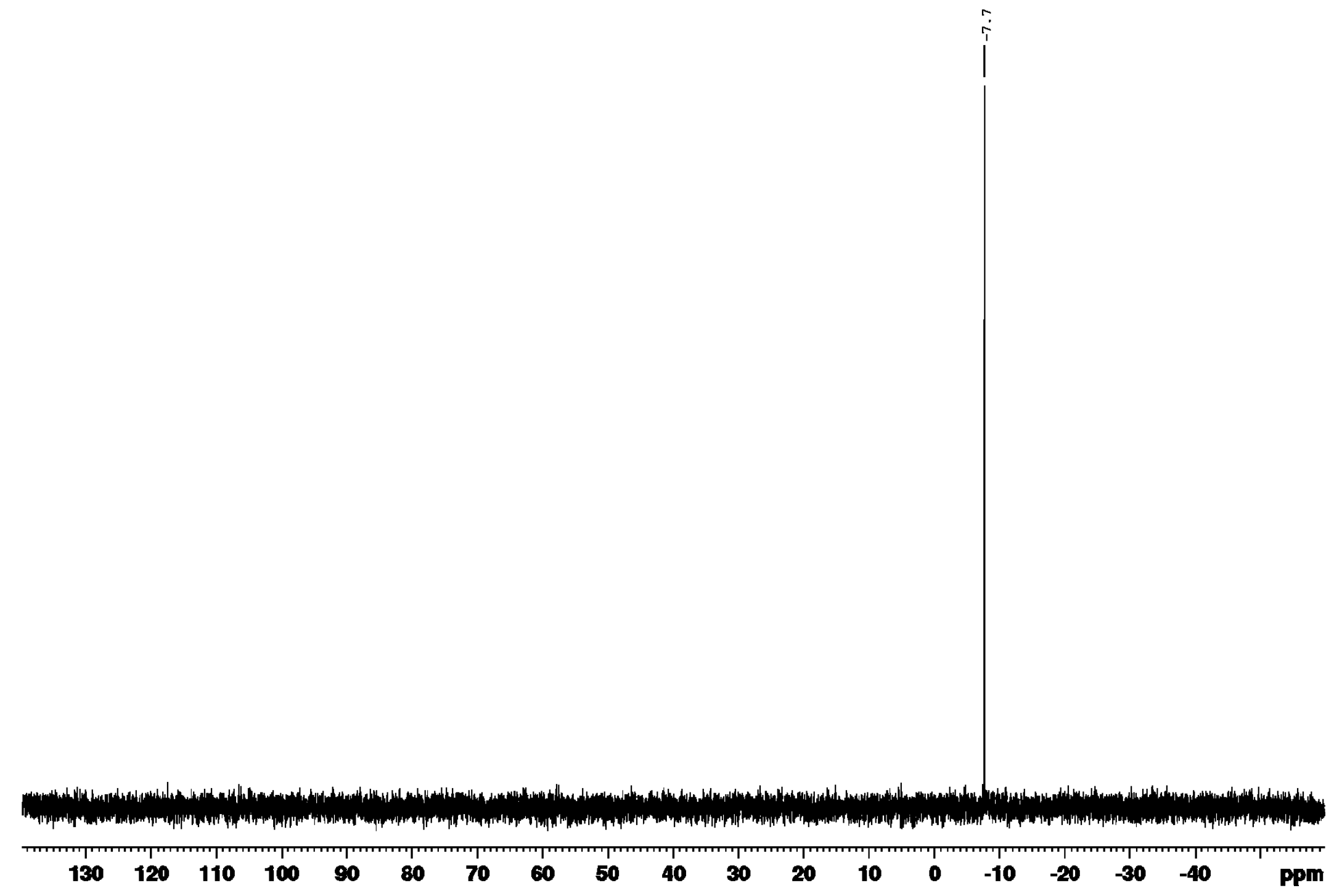


Figure S12. ${ }^{1} \mathrm{H}$ NMR $\left(500 \mathrm{MHz}, 1,2-\mathrm{Cl}_{2} \mathrm{C}_{6} \mathrm{D}_{4}\right)$ of $(S, R S)-5$.

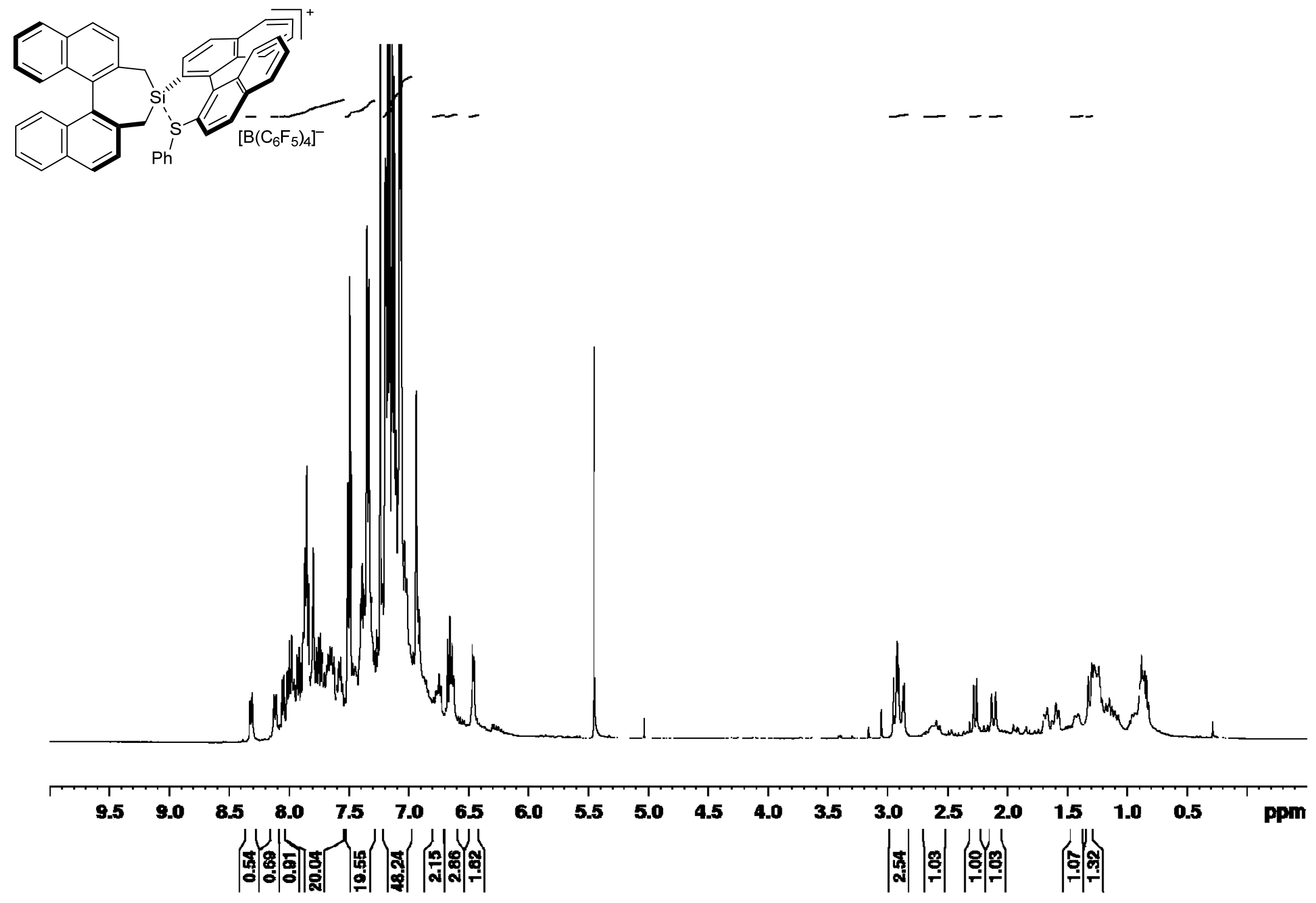


Figure S13. ${ }^{13} \mathrm{C}$ NMR $\left(126 \mathrm{MHz}, 1,2-\mathrm{Cl}_{2} \mathrm{C}_{6} \mathrm{D}_{4}\right)$ of $(S, R S)-5$.

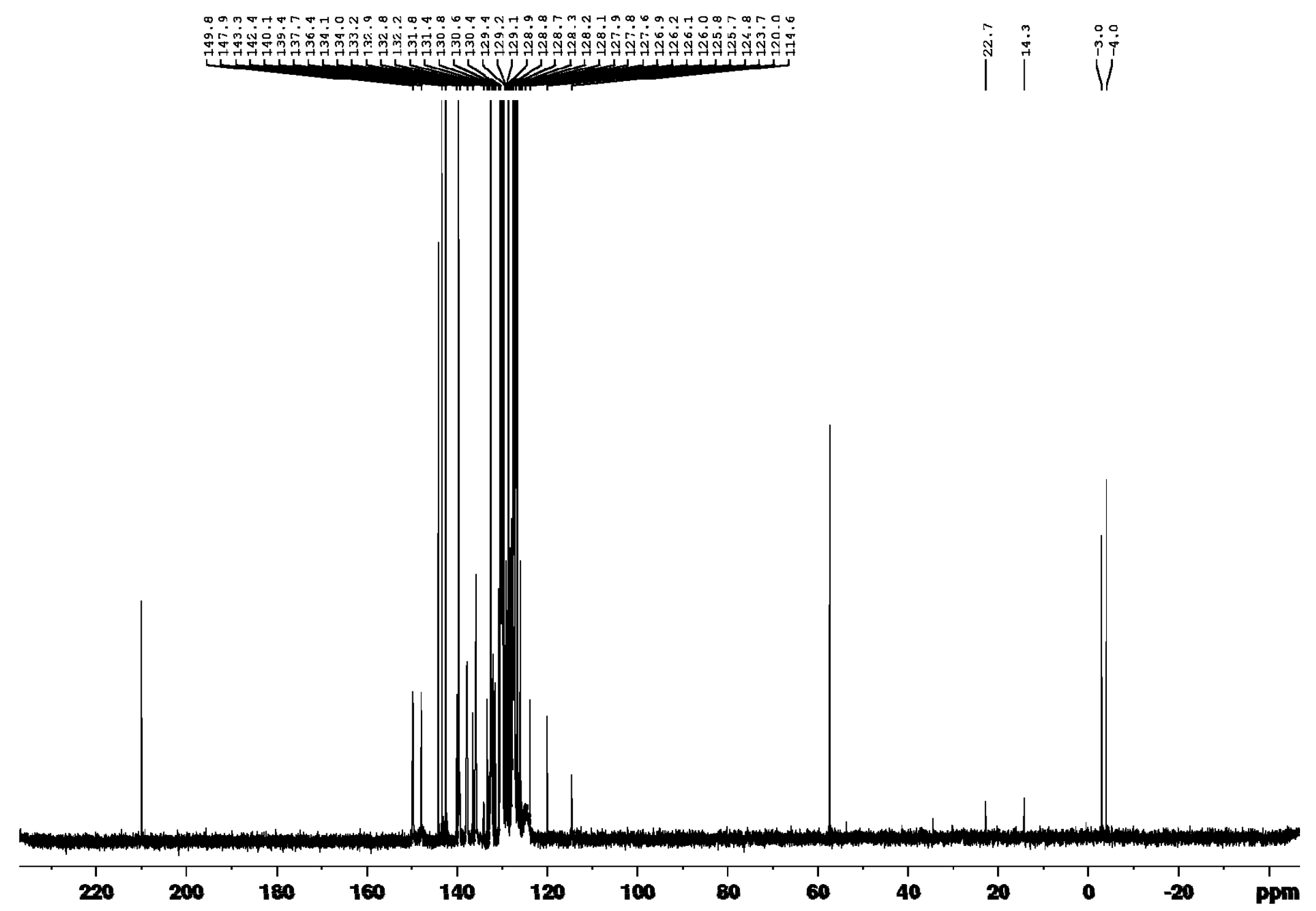


Figure S14. ${ }^{11} \mathrm{~B}$ NMR $\left(160 \mathrm{MHz}, 1,2-\mathrm{Cl}_{2} \mathrm{C}_{6} \mathrm{D}_{4}\right)$ of $(S, R S)-\mathbf{5}$.

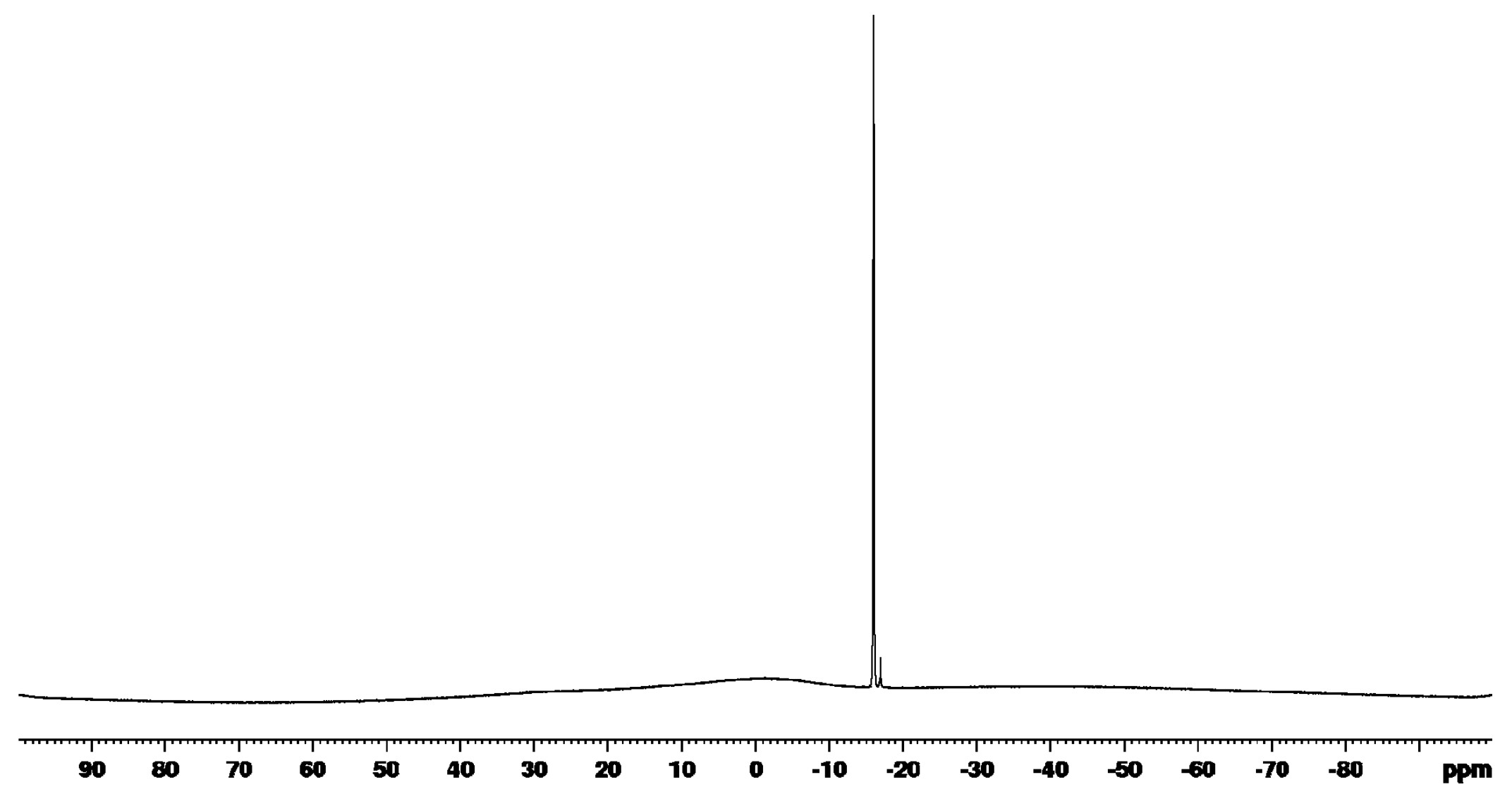


Figure S15. ${ }^{19} \mathrm{~F}$ NMR $\left(471 \mathrm{MHz}, 1,2-\mathrm{Cl}_{2} \mathrm{C}_{6} \mathrm{D}_{4}\right)$ of $(S, R S)-5$.

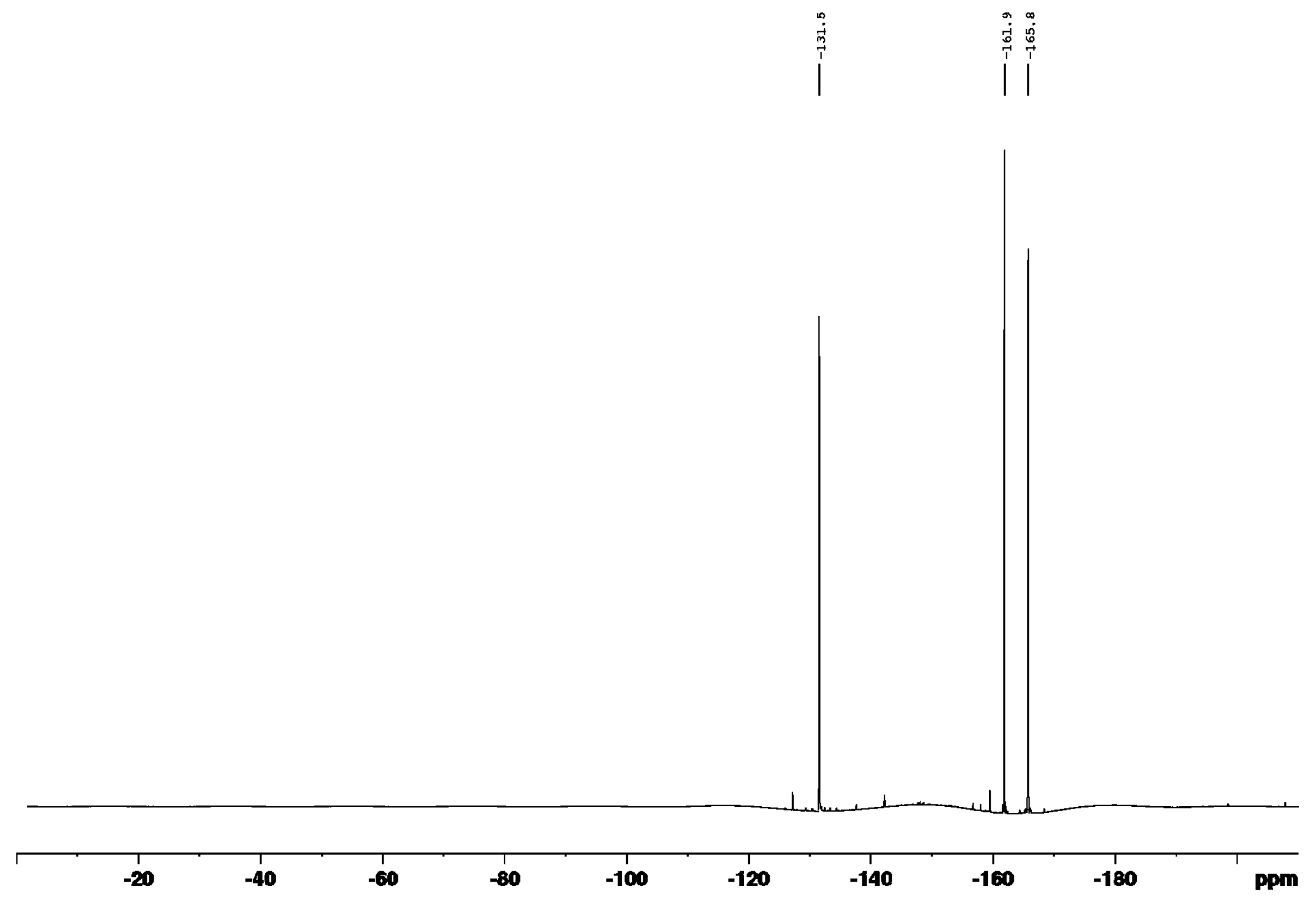


Figure S16. ${ }^{1} \mathrm{H},{ }^{29} \mathrm{Si} \mathrm{HMQC} \mathrm{NMR}\left(500 / 99 \mathrm{MHz}, 1,2-\mathrm{Cl}_{2} \mathrm{C}_{6} \mathrm{D}_{4}\right)$ of $(S, R S)-5$.

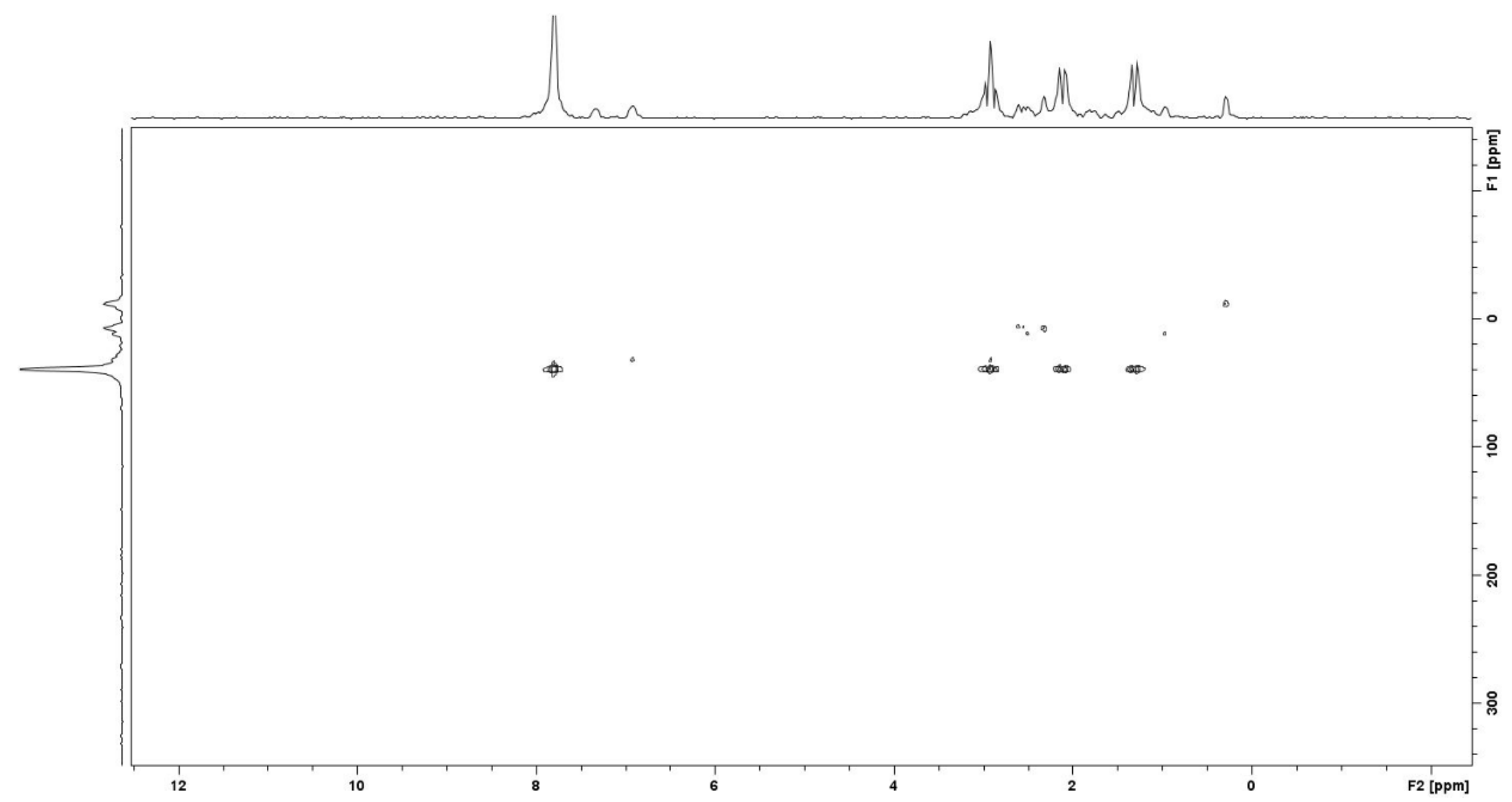


Figure S17. ${ }^{1} \mathrm{H}$ NMR $\left(500 \mathrm{MHz}, 1,2-\mathrm{Cl}_{2} \mathrm{C}_{6} \mathrm{D}_{4}\right)$ of $(S, S)-\mathbf{5}$.

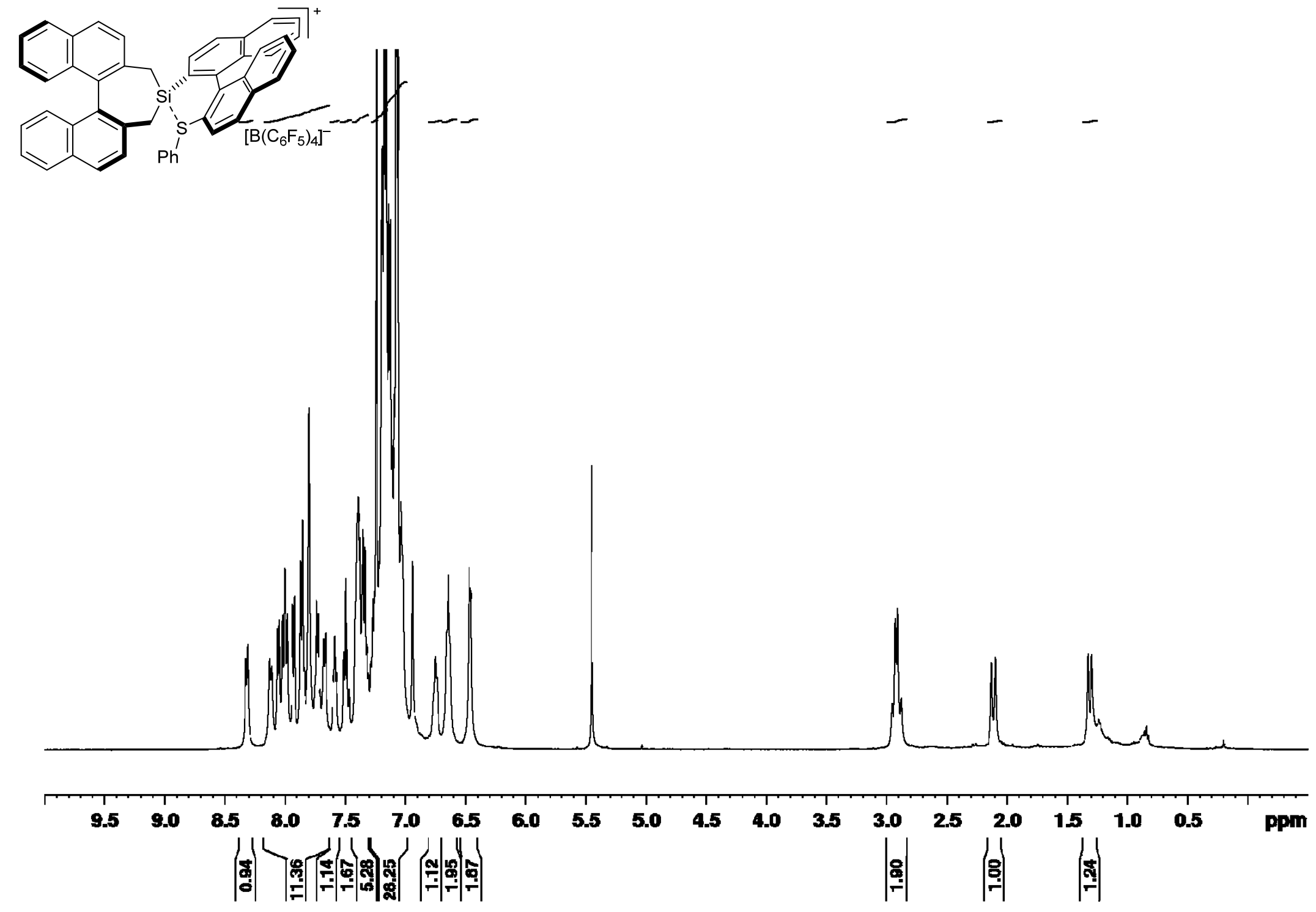


Figure S18. ${ }^{13} \mathrm{C}$ NMR $\left(126 \mathrm{MHz}, 1,2-\mathrm{Cl}_{2} \mathrm{C}_{6} \mathrm{D}_{4}\right)$ of $(S, S)-5$.

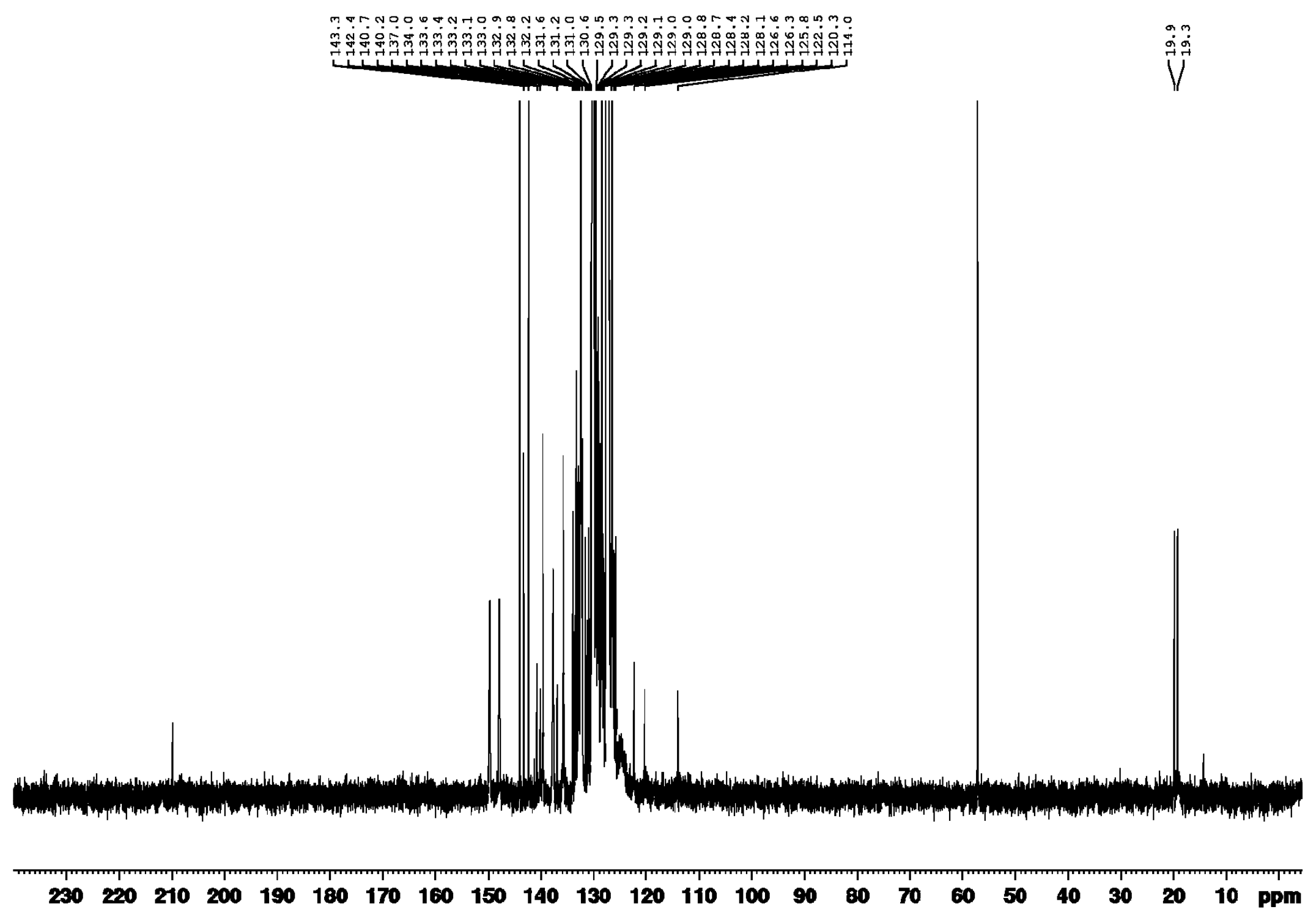


Figure S19. ${ }^{11} \mathrm{~B}$ NMR (160 MHz, $\left.1,2-\mathrm{Cl}_{2} \mathrm{C}_{6} \mathrm{D}_{4}\right)$ of $(S, S)-5$.

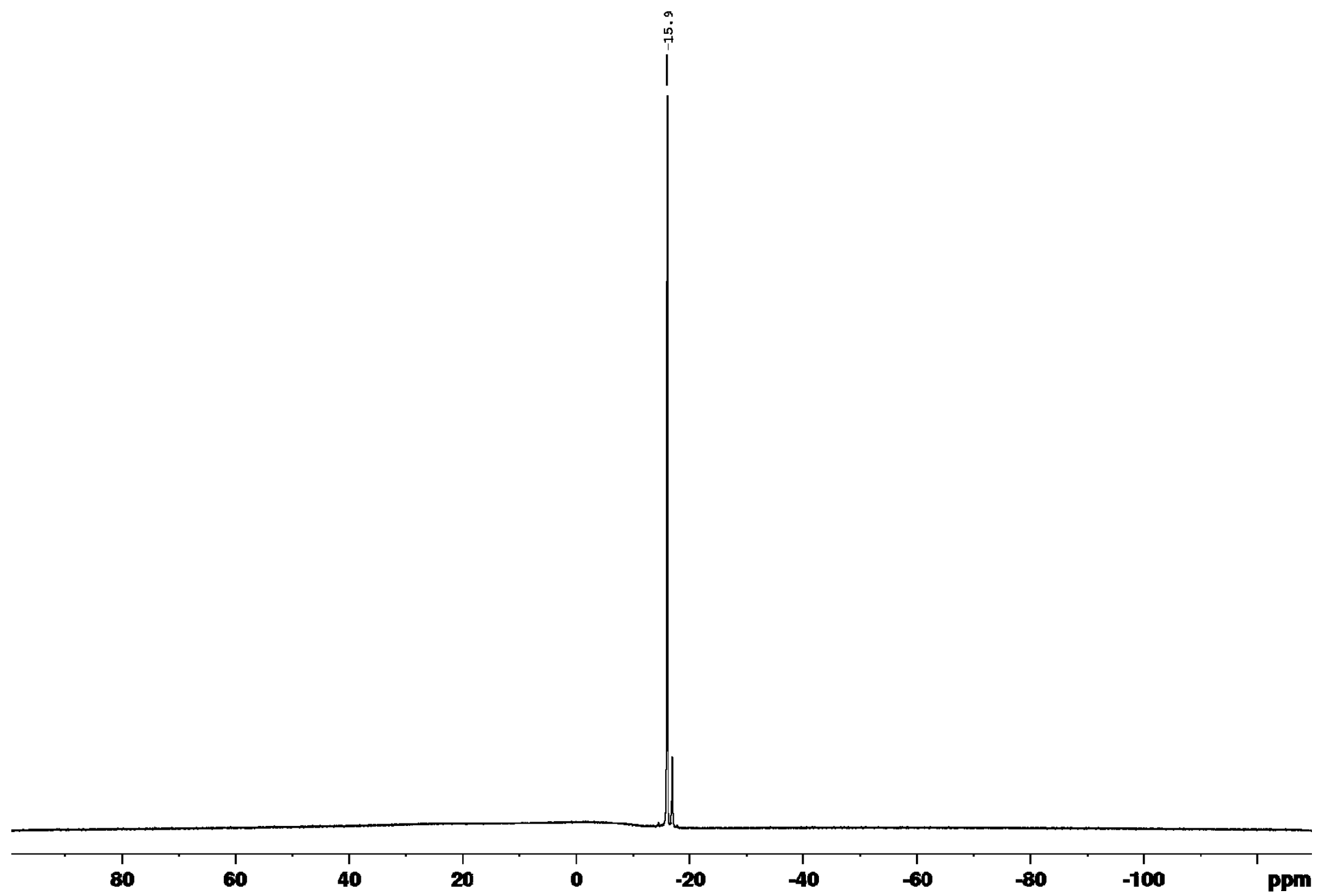


Figure S20. ${ }^{19} \mathrm{~F}$ NMR $\left(471 \mathrm{MHz}, 1,2-\mathrm{Cl}_{2} \mathrm{C}_{6} \mathrm{D}_{4}\right)$ of $(S, S)-5$.

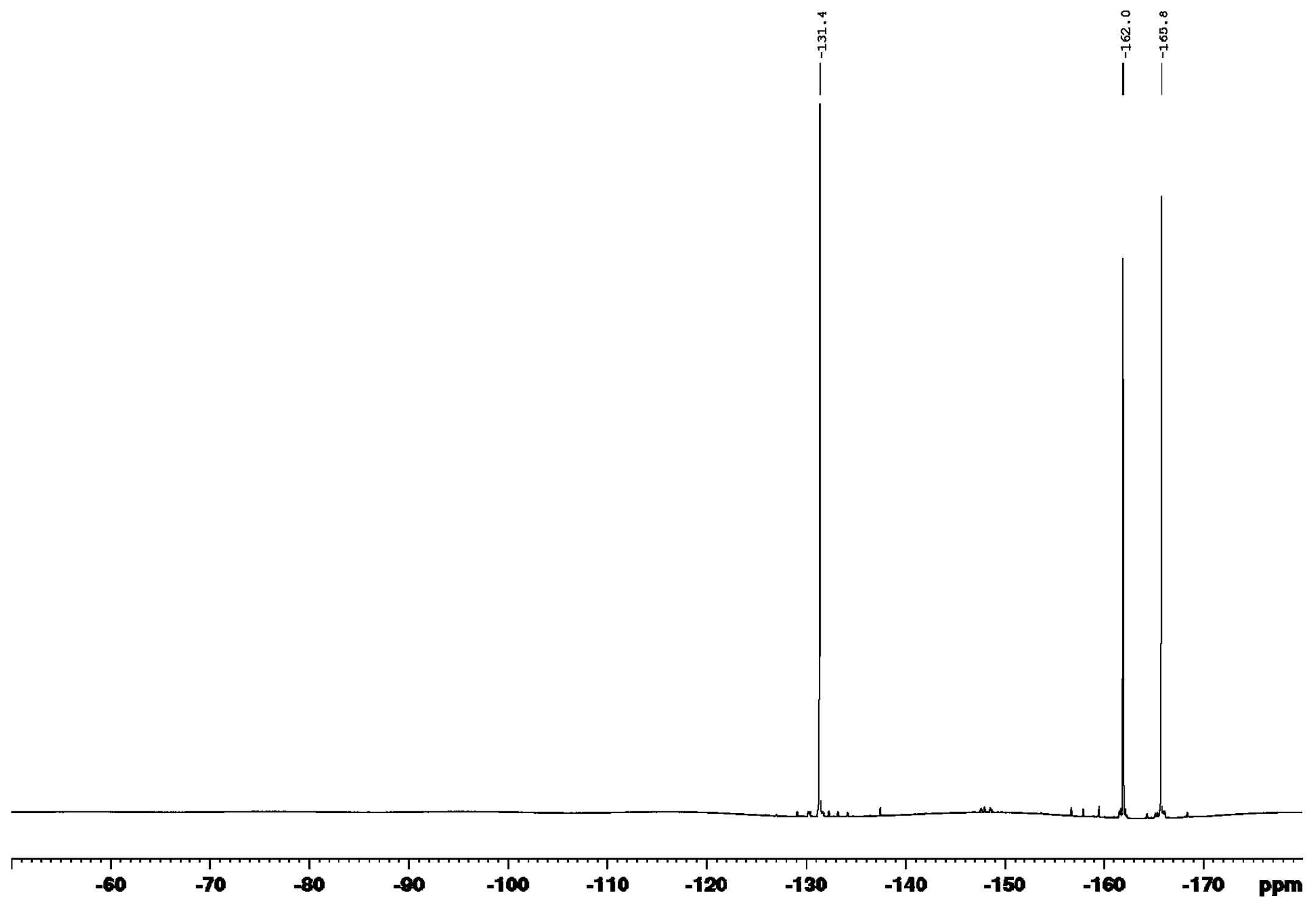


Supporting Information for Organometallic

S51

Figure S21. ${ }^{29}$ Si DEPT NMR $\left(99 \mathrm{MHz}, 1,2-\mathrm{Cl}_{2} \mathrm{C}_{6} \mathrm{D}_{4}\right)$ of $(S, S)-5$.<smiles>C[Te][Te]</smiles>

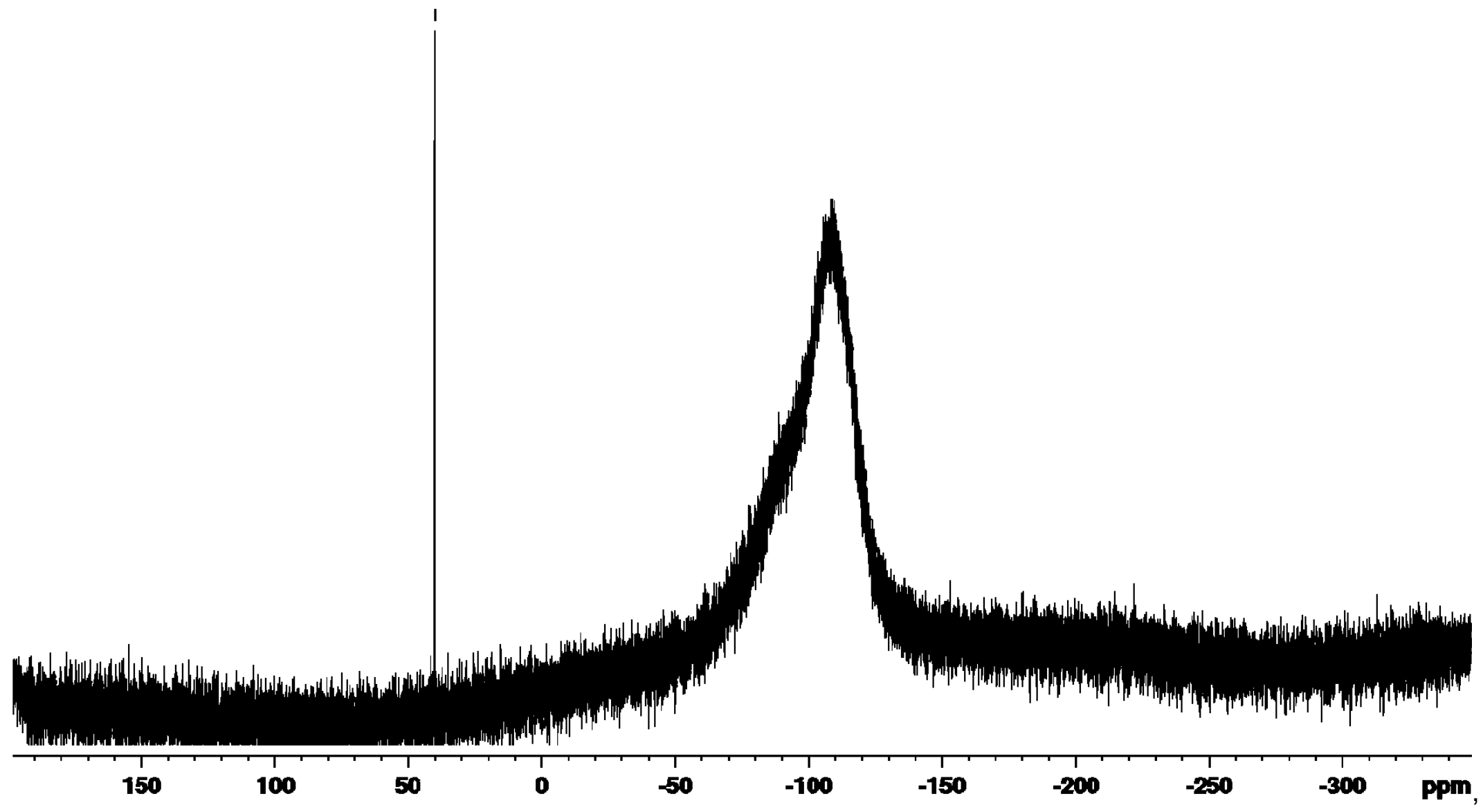


Figure S22. ${ }^{1} \mathrm{H},{ }^{29} \mathrm{Si} \mathrm{HMQC} \mathrm{NMR}\left(500 / 99 \mathrm{MHz}, 1,2-\mathrm{Cl}_{2} \mathrm{C}_{6} \mathrm{D}_{4}\right)$ of $(\mathrm{S}, \mathrm{S})-\mathbf{5}$.

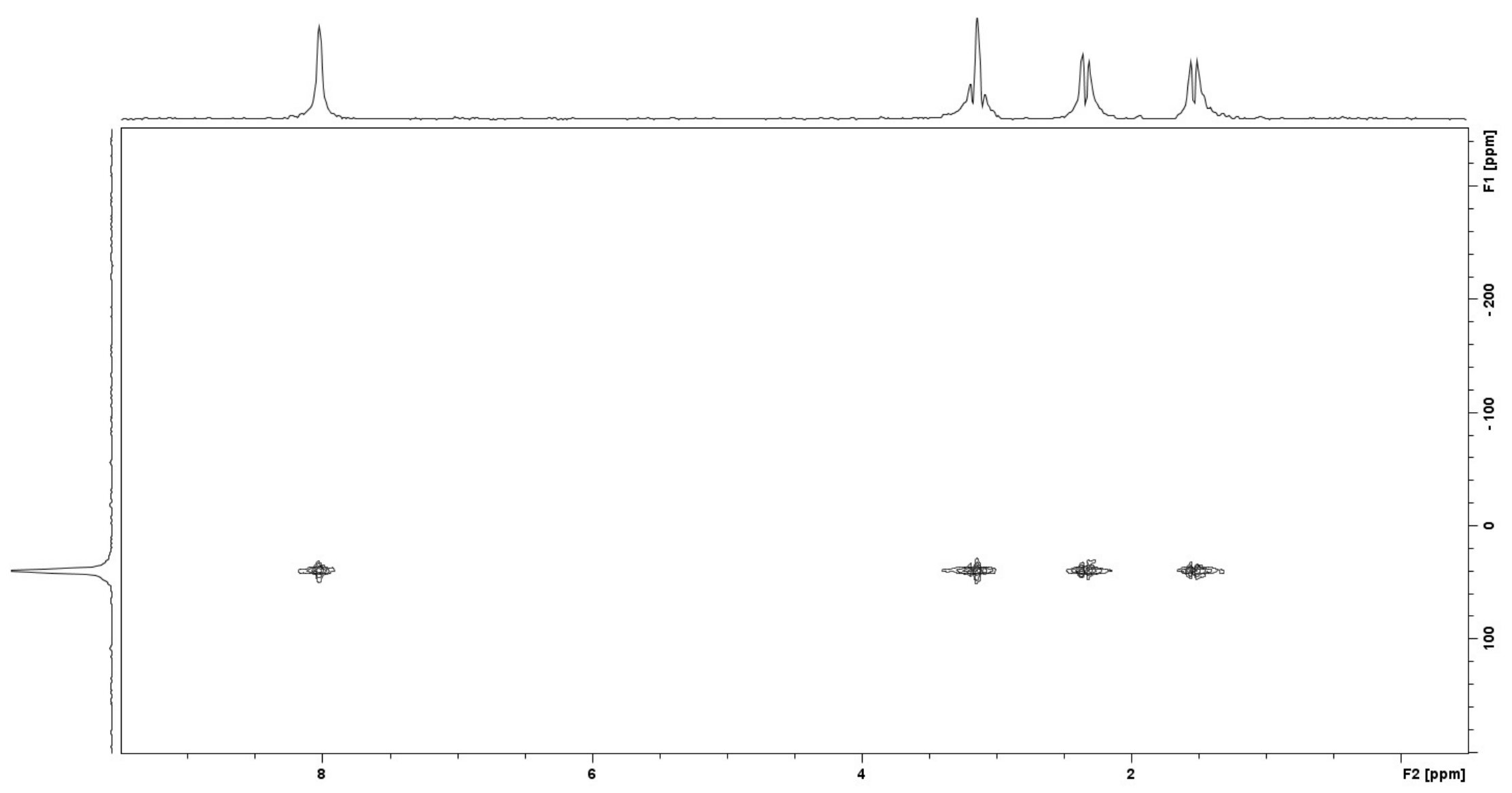




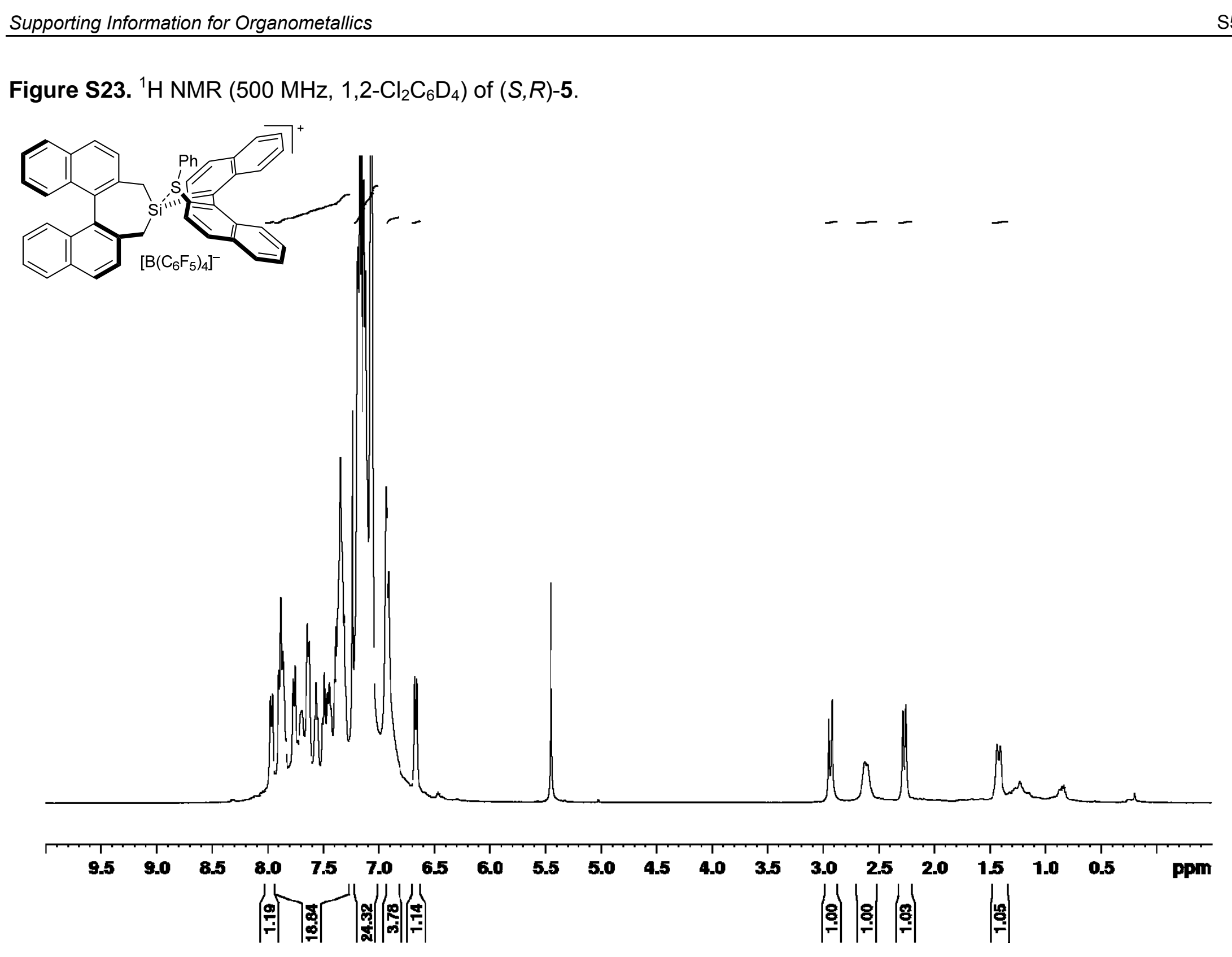


Figure S24. ${ }^{13} \mathrm{C}$ NMR $\left(126 \mathrm{MHz}, 1,2-\mathrm{Cl}_{2} \mathrm{C}_{6} \mathrm{D}_{4}\right)$ of $(S, R)-\mathbf{5}$.

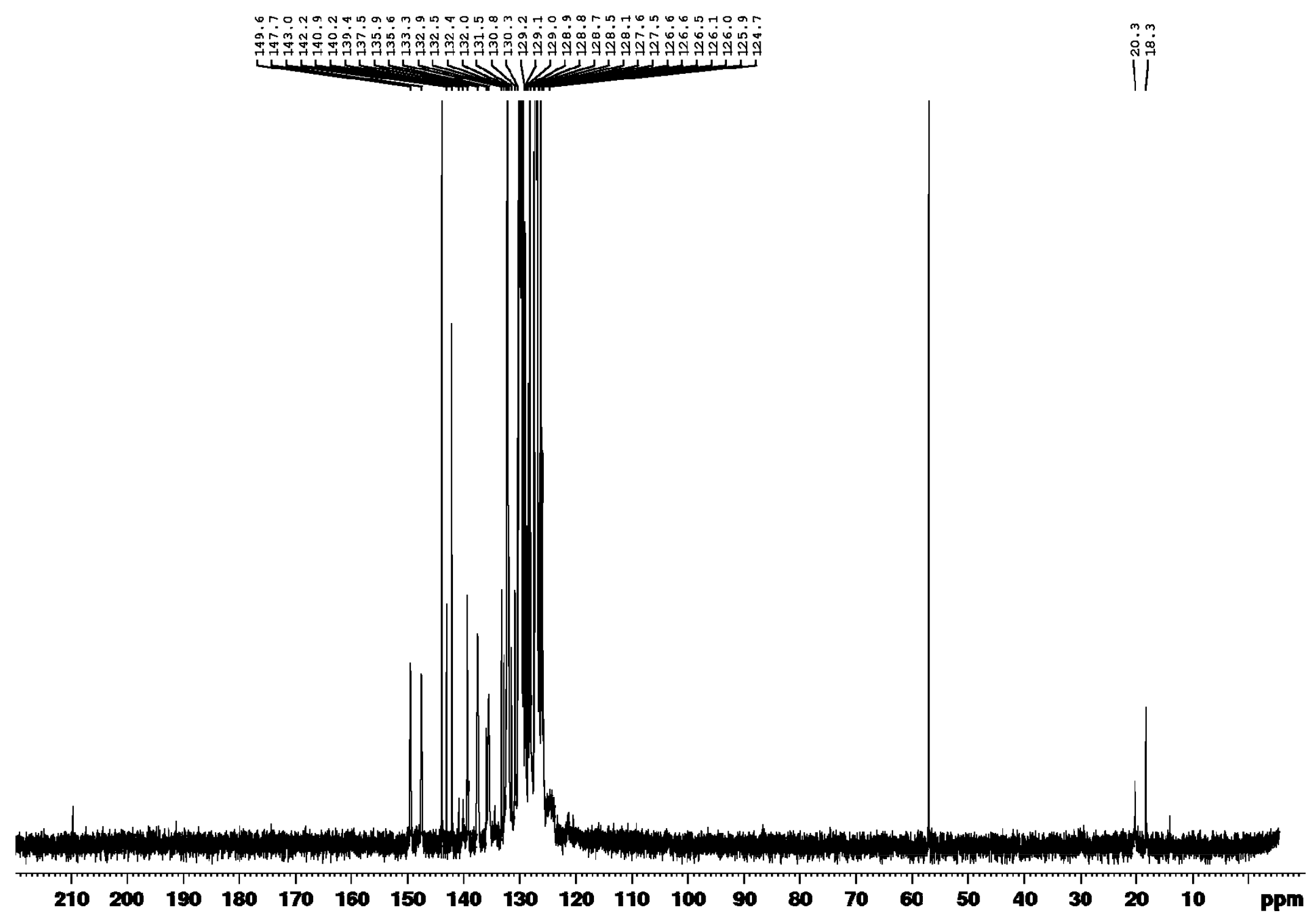


Figure S25. ${ }^{11} \mathrm{~B}$ NMR $\left(160 \mathrm{MHz}, 1,2-\mathrm{Cl}_{2} \mathrm{C}_{6} \mathrm{D}_{4}\right)$ of $(S, R)-5$.

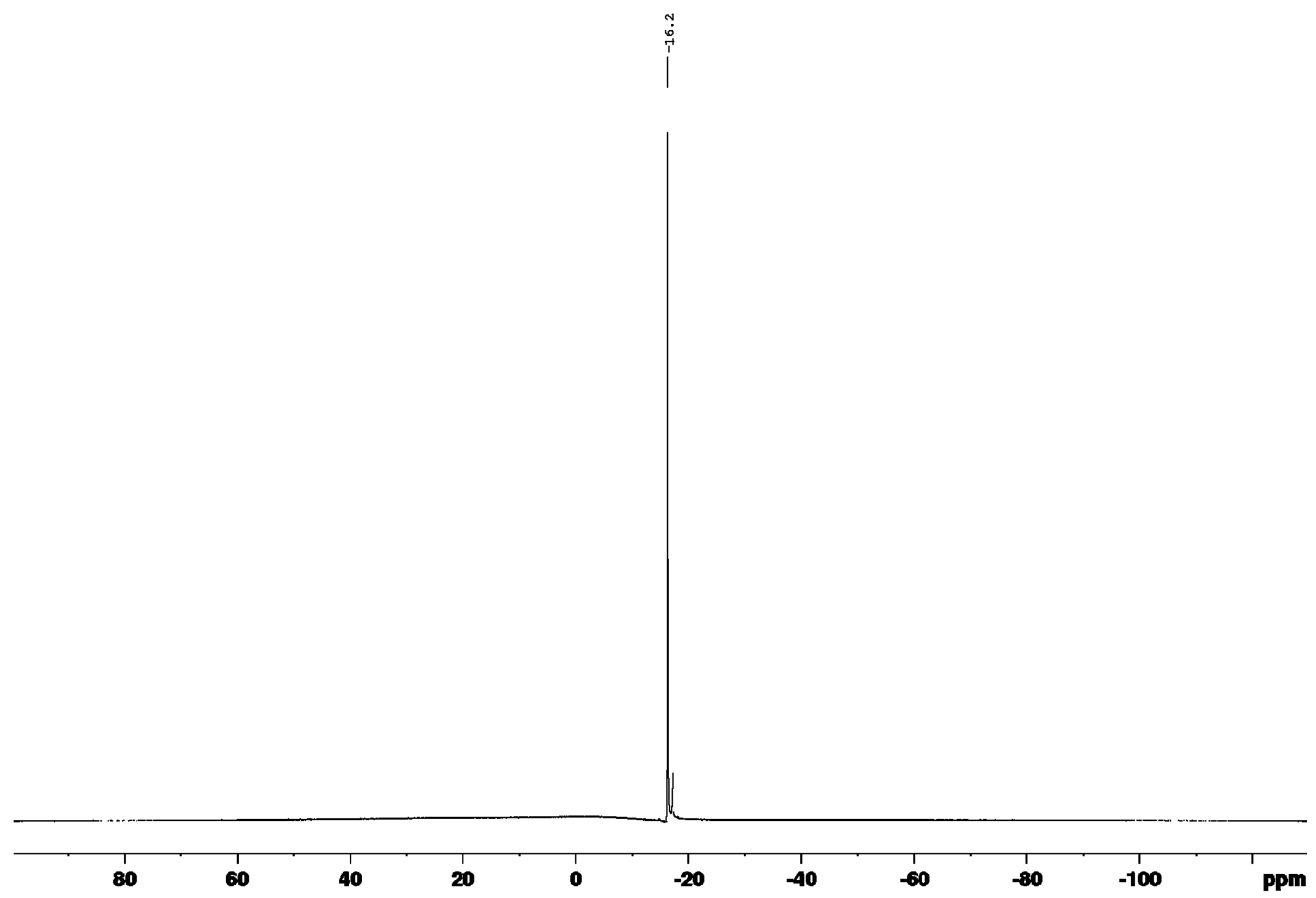


Figure S26. ${ }^{19} \mathrm{~F}$ NMR $\left(471 \mathrm{MHz}, 1,2-\mathrm{Cl}_{2} \mathrm{C}_{6} \mathrm{D}_{4}\right)$ of $(S, R)-\mathbf{5}$.

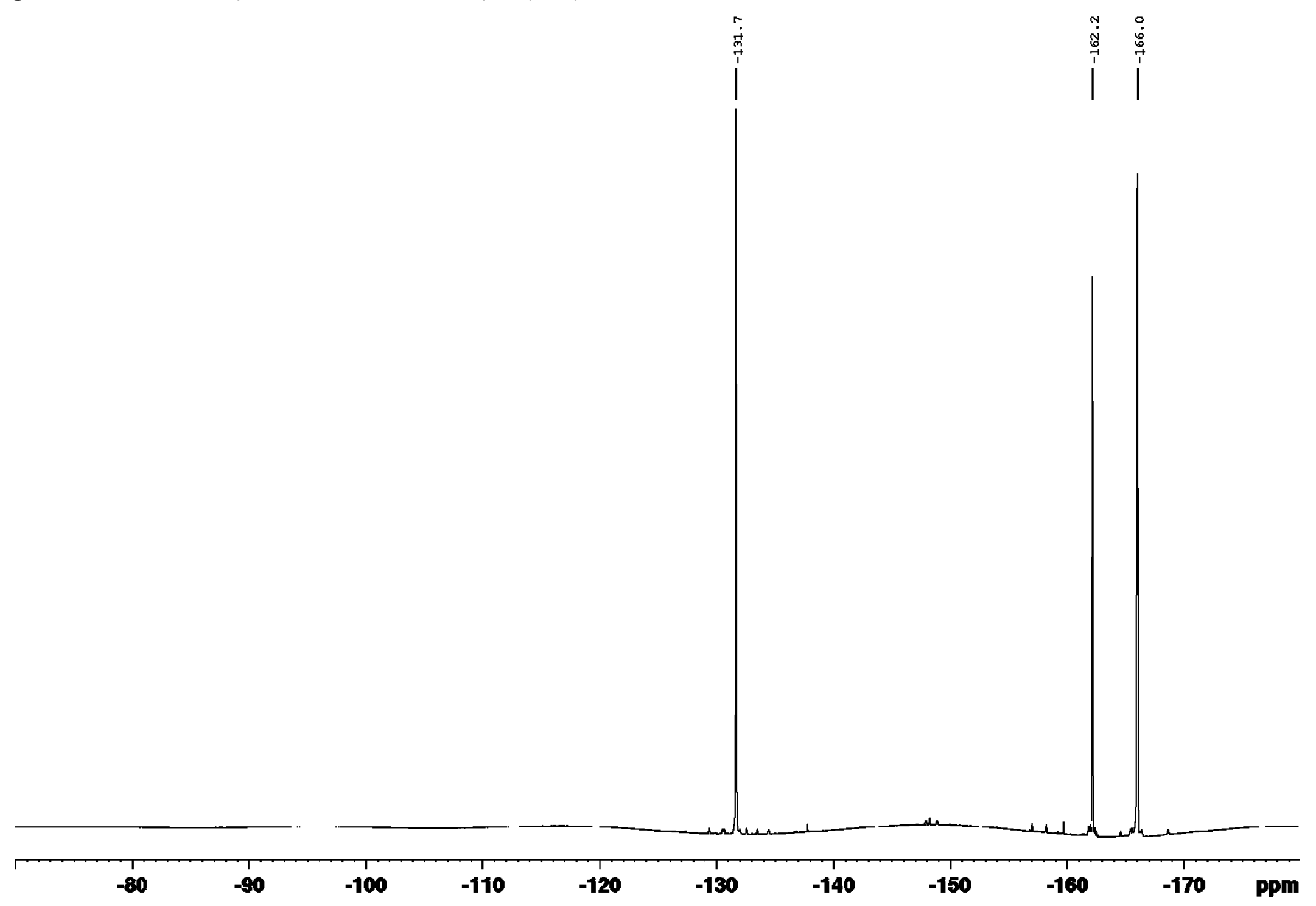


Figure S27. ${ }^{1} \mathrm{H},{ }^{29} \mathrm{Si} \mathrm{HMQC} \mathrm{NMR}\left(500 / 99 \mathrm{MHz}, 1,2-\mathrm{Cl}_{2} \mathrm{C}_{6} \mathrm{D}_{4}\right)$ of $(S, R)-5$.

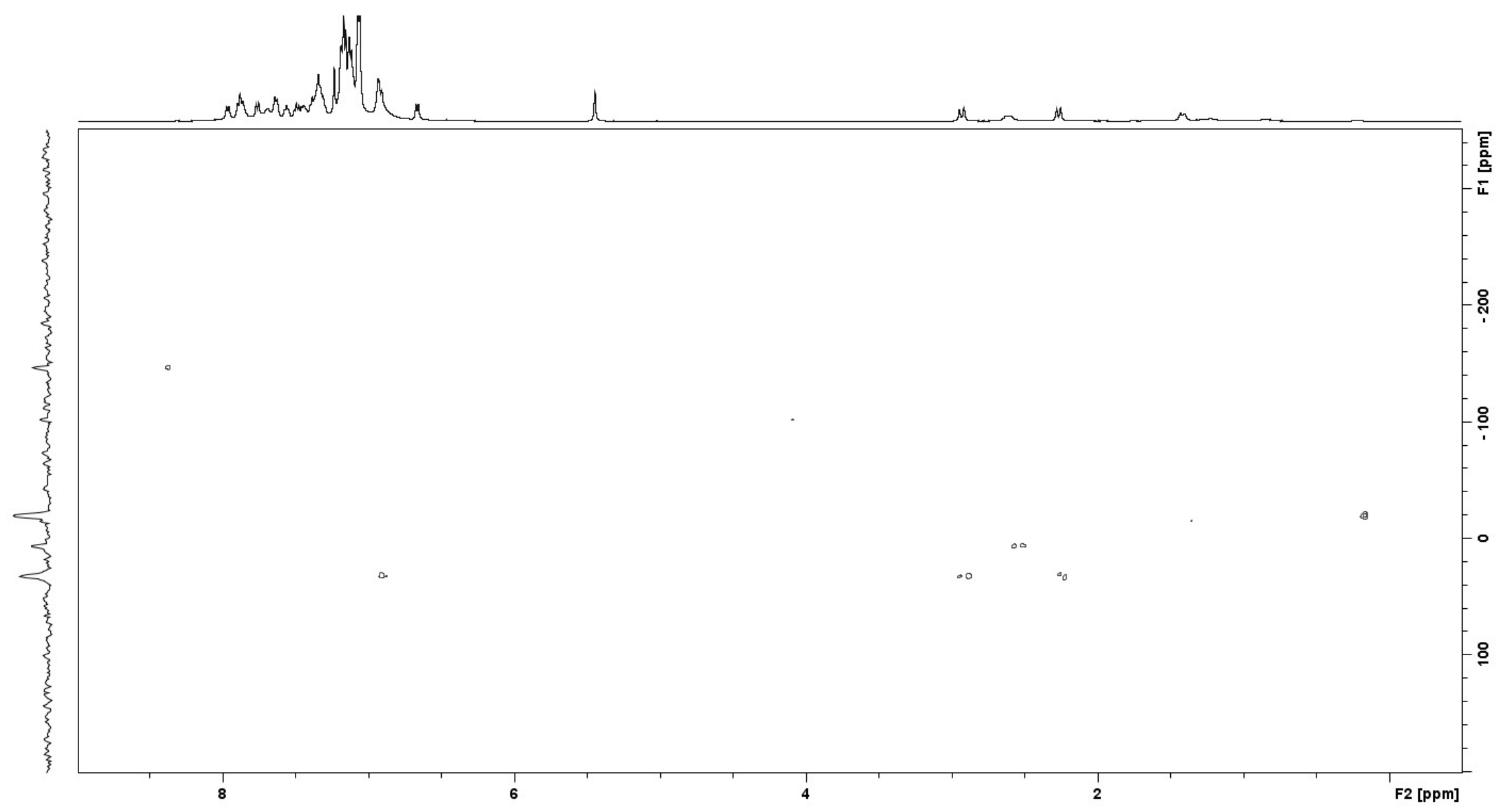


Figure S28. ${ }^{1} \mathrm{H} \mathrm{NMR}\left(500 \mathrm{MHz}, 1,2-\mathrm{Cl}_{2} \mathrm{C}_{6} \mathrm{D}_{4}\right)$ of $(S, R)-5 \cdot \mathrm{O}=\mathrm{CPh}_{2}$.

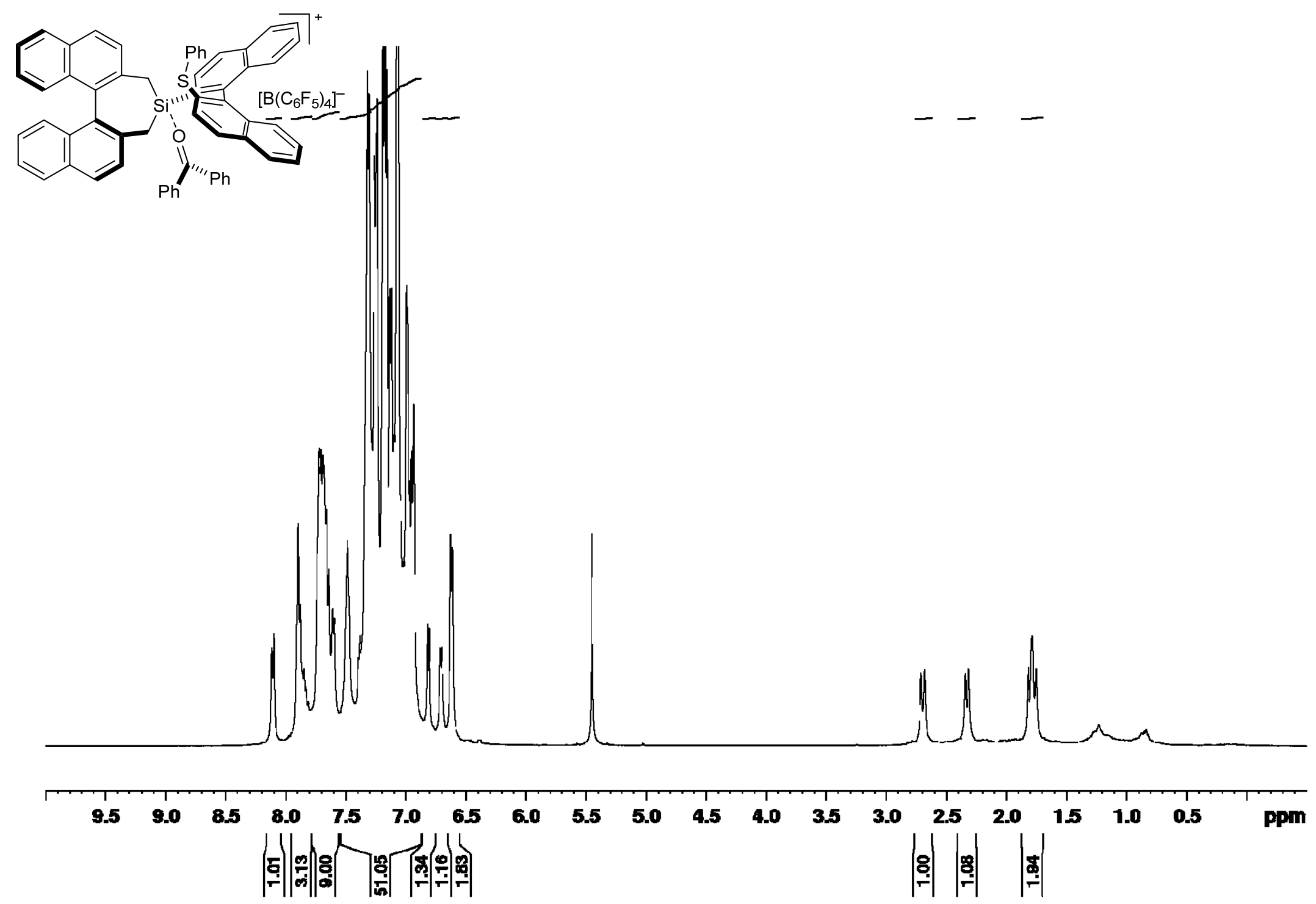


Figure S29. ${ }^{13} \mathrm{C}$ NMR $\left(126 \mathrm{MHz}, 1,2-\mathrm{Cl}_{2} \mathrm{C}_{6} \mathrm{D}_{4}\right)$ of $(\mathrm{S}, R)-\mathbf{5} \cdot \mathrm{O}=\mathrm{CPh}_{2}$.

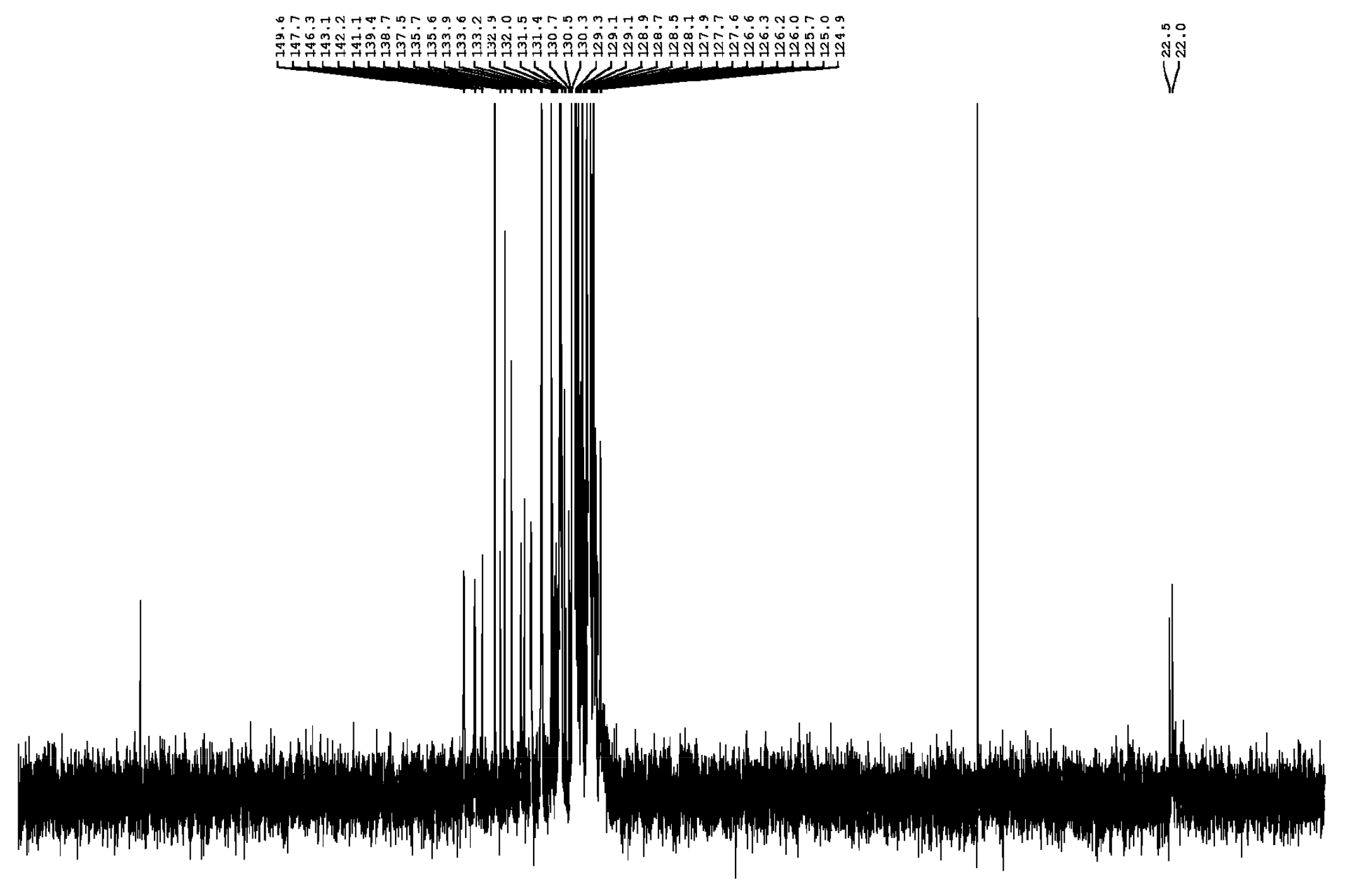

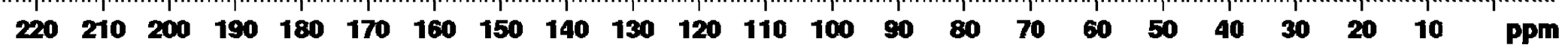


Figure S30. ${ }^{11} \mathrm{~B}$ NMR $\left(160 \mathrm{MHz}, 1,2-\mathrm{Cl}_{2} \mathrm{C}_{6} \mathrm{D}_{4}\right)$ of $(\mathrm{S}, R)-\mathbf{5} \cdot \mathrm{O}=\mathrm{CPh}_{2}$.

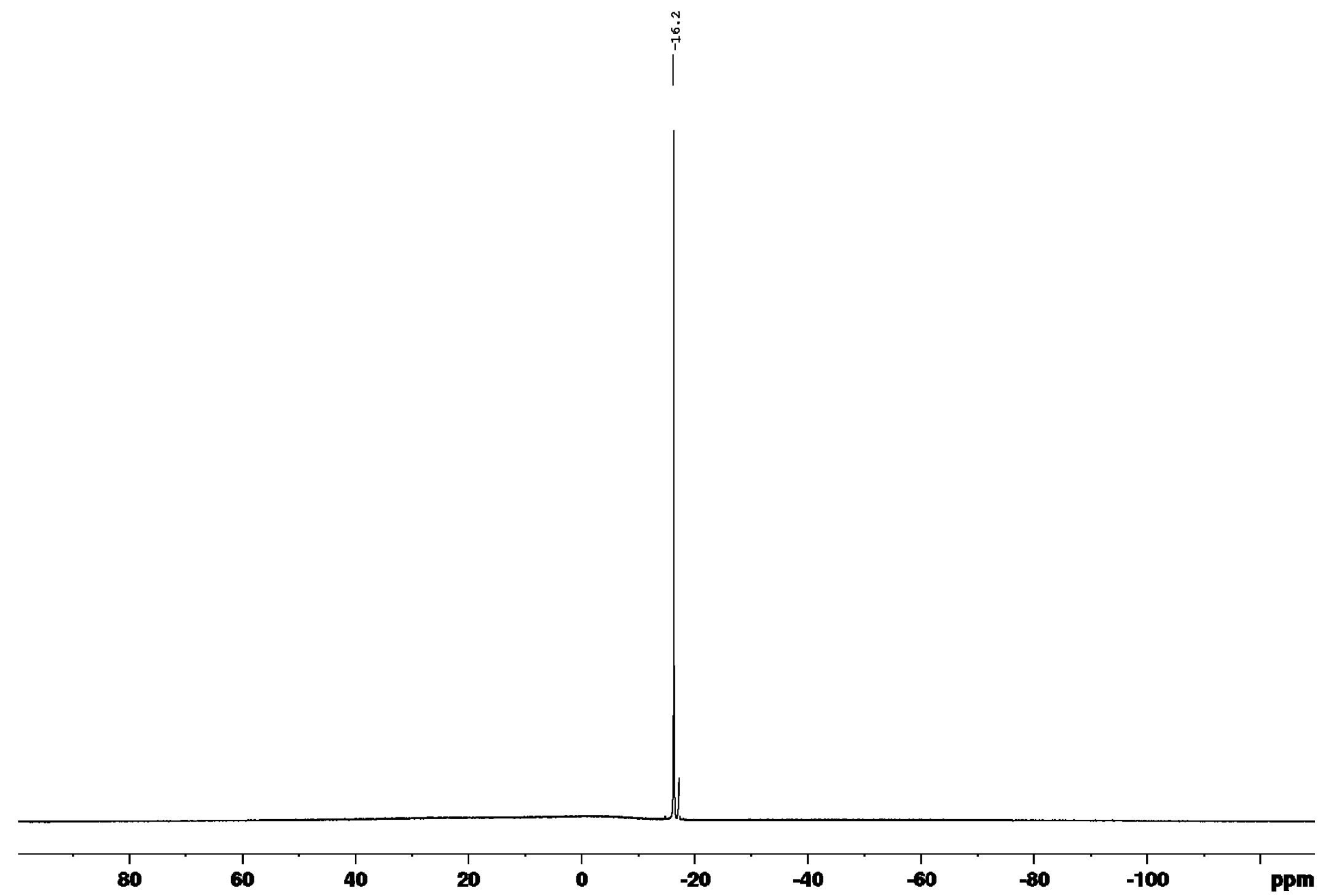


Figure S31. ${ }^{19} \mathrm{~F}$ NMR $\left(471 \mathrm{MHz}, 1,2-\mathrm{Cl}_{2} \mathrm{C}_{6} \mathrm{D}_{4}\right)$ of $(\mathrm{S}, R)-\mathbf{5} \cdot \mathrm{O}=\mathrm{CPh}_{2}$.

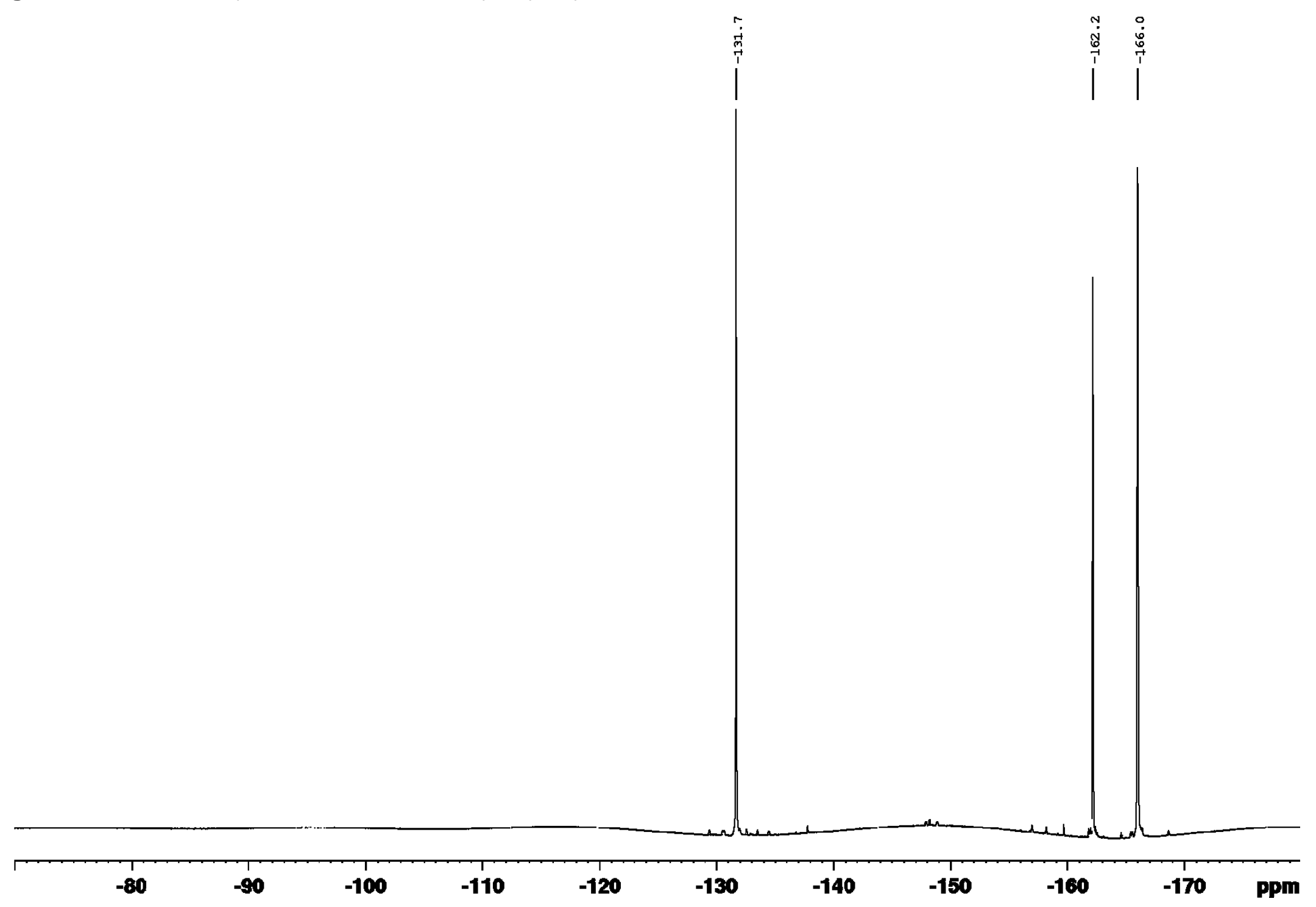


Figure S32. ${ }^{29} \mathrm{Si}$ DEPT NMR $\left(99 \mathrm{MHz}, 1,2-\mathrm{Cl}_{2} \mathrm{C}_{6} \mathrm{D}_{4}\right)$ of $(\mathrm{S}, R)-5 \cdot \mathrm{O}=\mathrm{CPh}_{2}$.

$i^{m}$

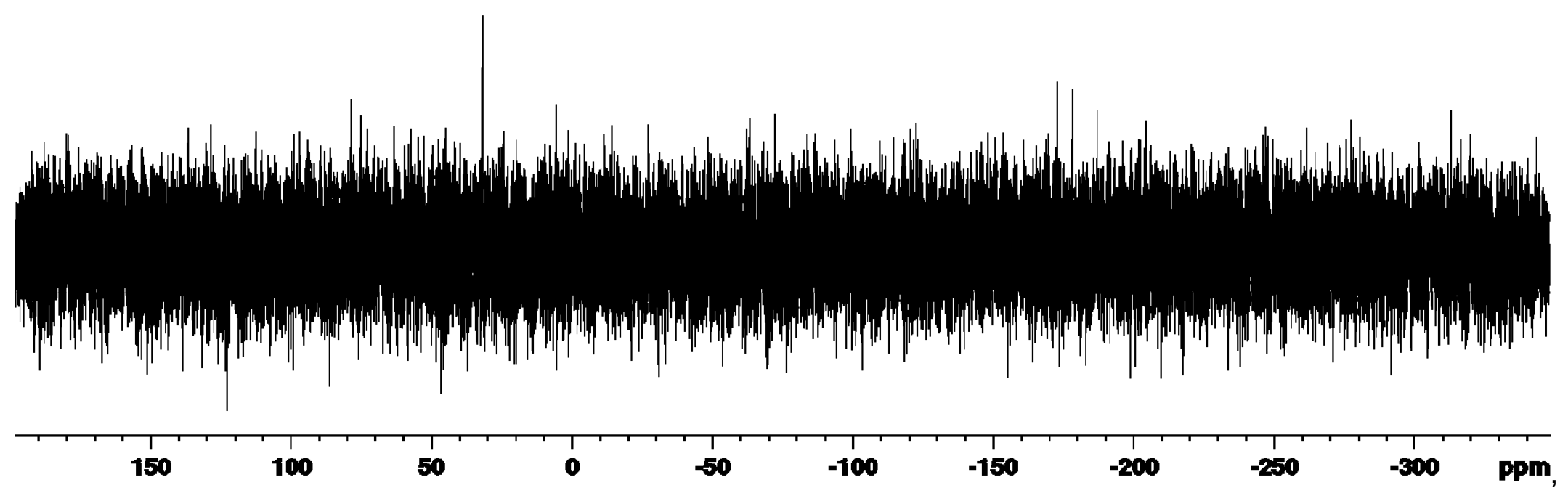


Figure S33. ${ }^{1} \mathrm{H},{ }^{29} \mathrm{Si} \mathrm{HMQC} \mathrm{NMR}\left(500 / 99 \mathrm{MHz}, 1,2-\mathrm{Cl}_{2} \mathrm{C}_{6} \mathrm{D}_{4}\right)$ of $(S, R)-5 \cdot \mathrm{O}=\mathrm{CPh}_{2}$.

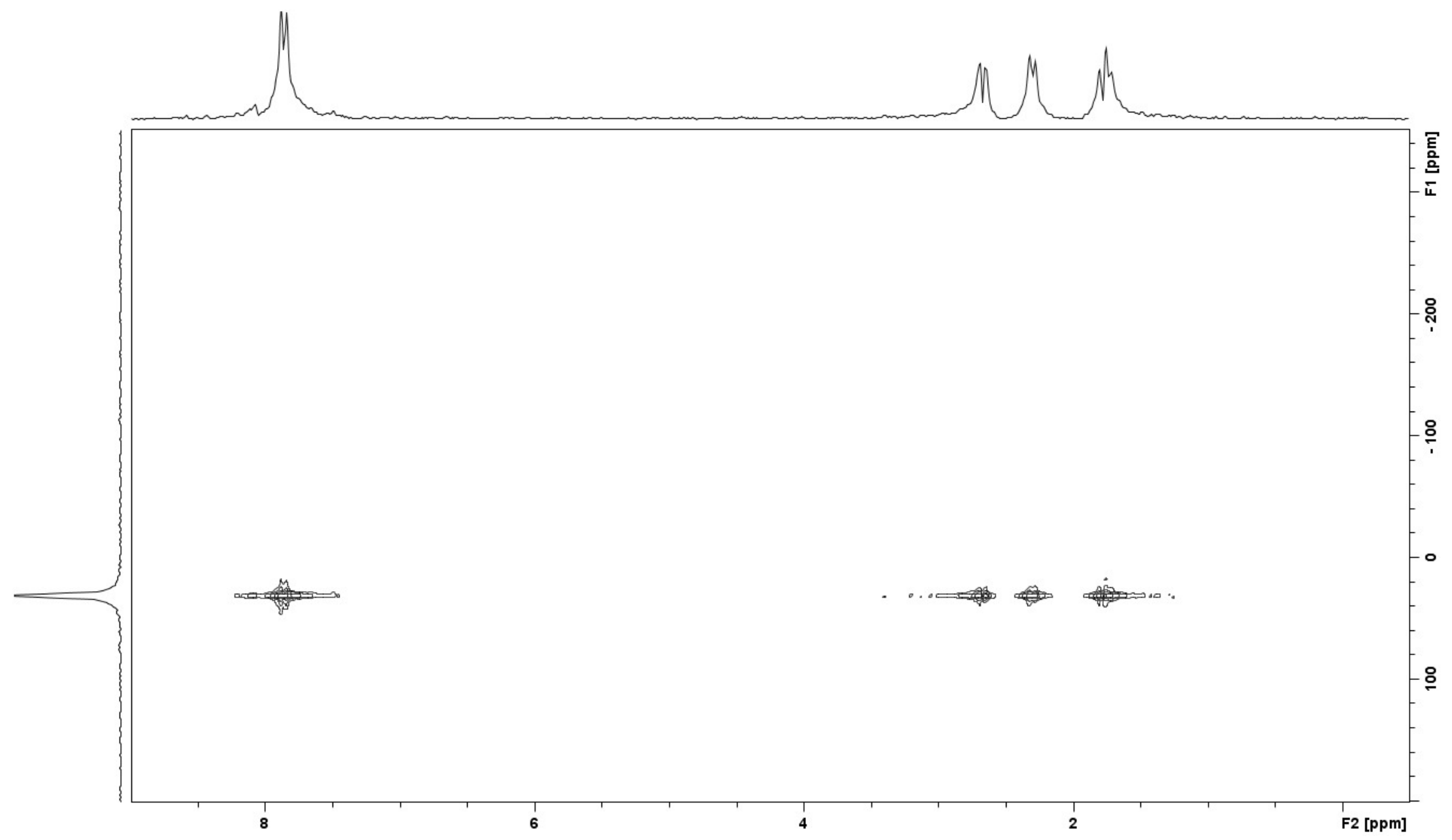


Supporting Information for Organometallics

S64

Figure S34. ${ }^{1} \mathrm{H}$ NMR (500 MHz, $\left.\mathrm{CDCl}_{3}\right)$ of 12.
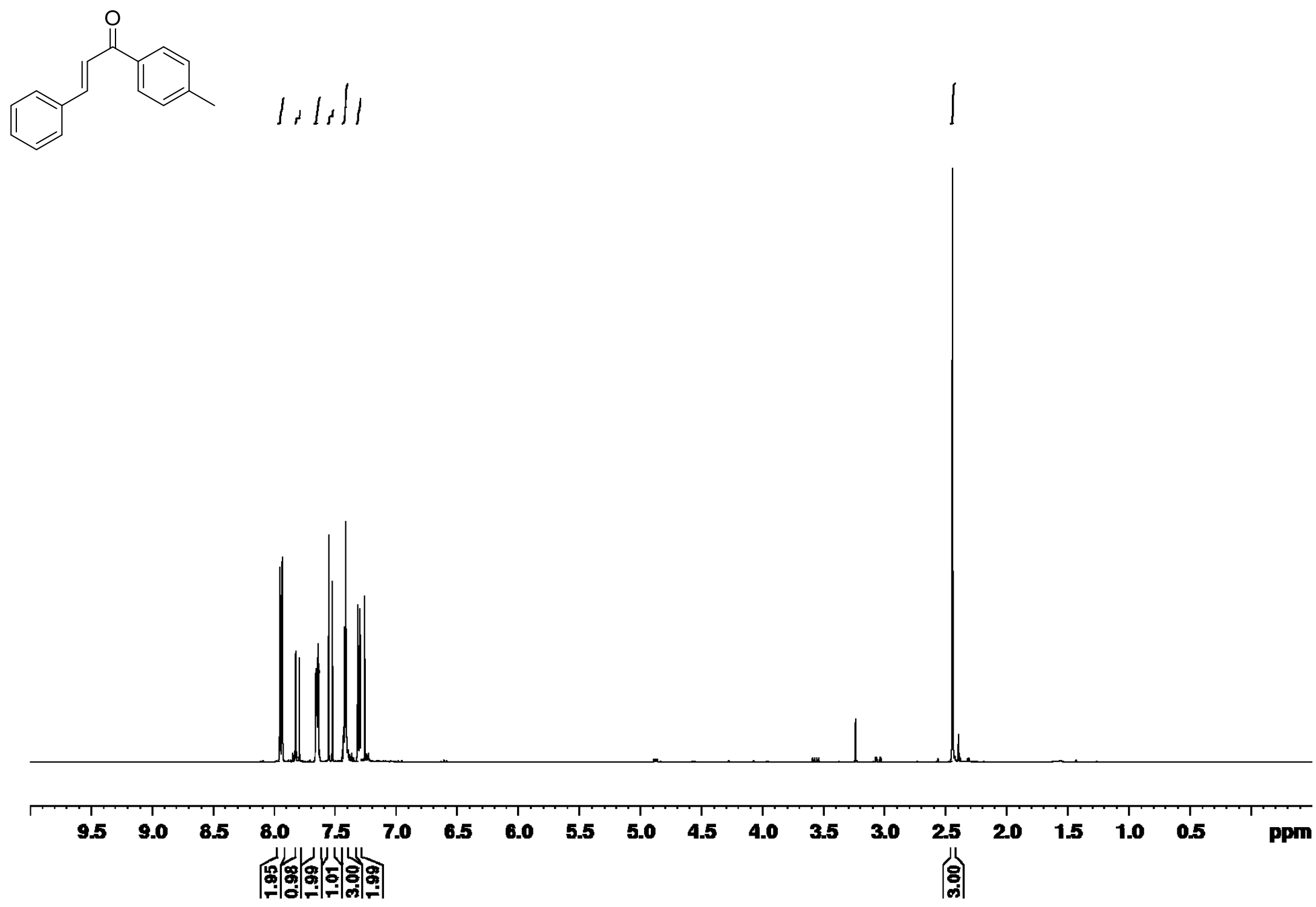
Figure S35. ${ }^{13} \mathrm{C}$ NMR $\left(126 \mathrm{MHz}, \mathrm{CDCl}_{3}\right)$ of 12 .
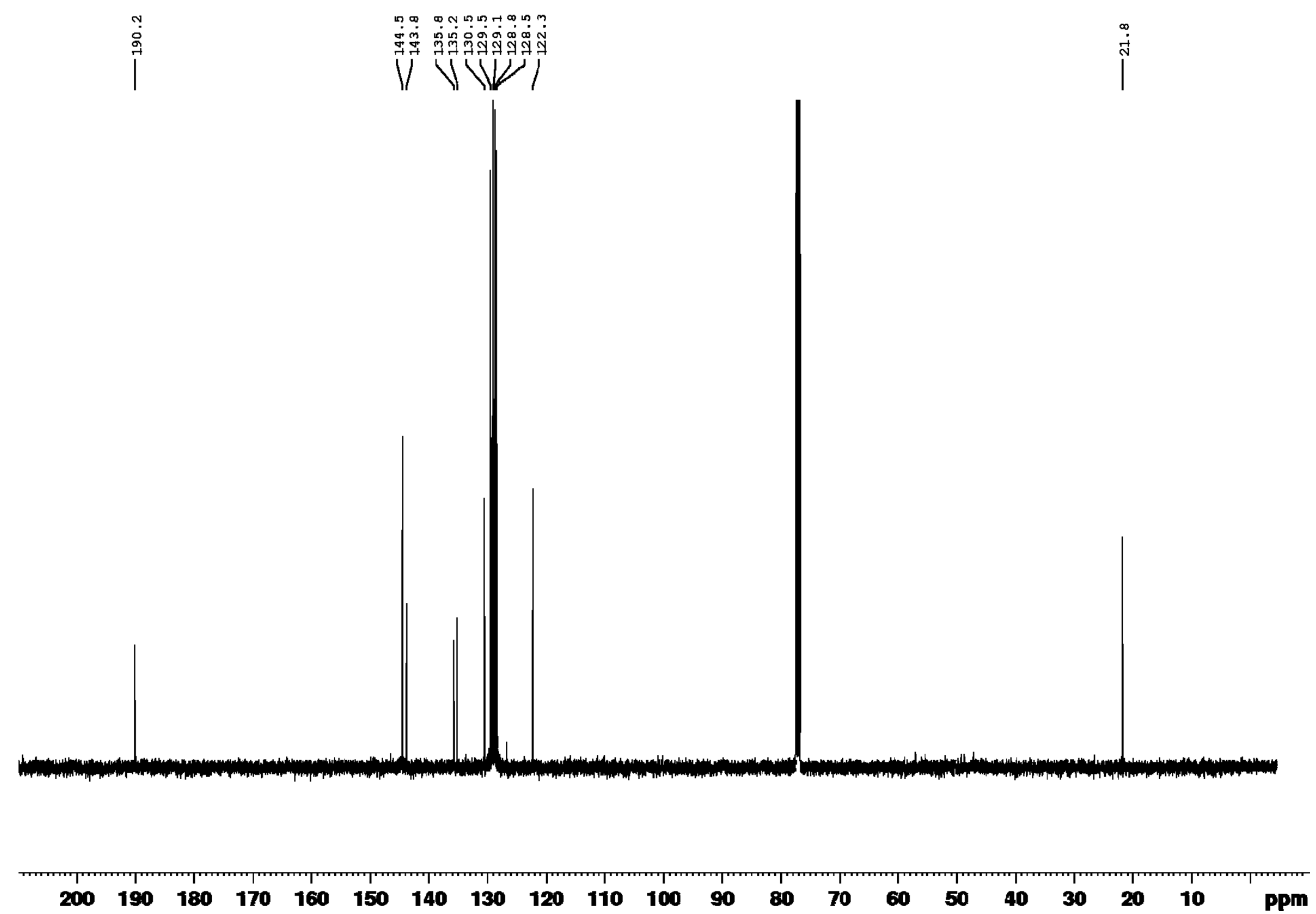
Supporting Information for Organometallic

S66

Figure S36. ${ }^{1} \mathrm{H}$ NMR $\left(500 \mathrm{MHz}, \mathrm{CDCl}_{3}\right)$ of 13.<smiles>CC(C)(C)c1ccc(C(=O)/C=C/c2ccccc2)cc1</smiles>

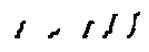
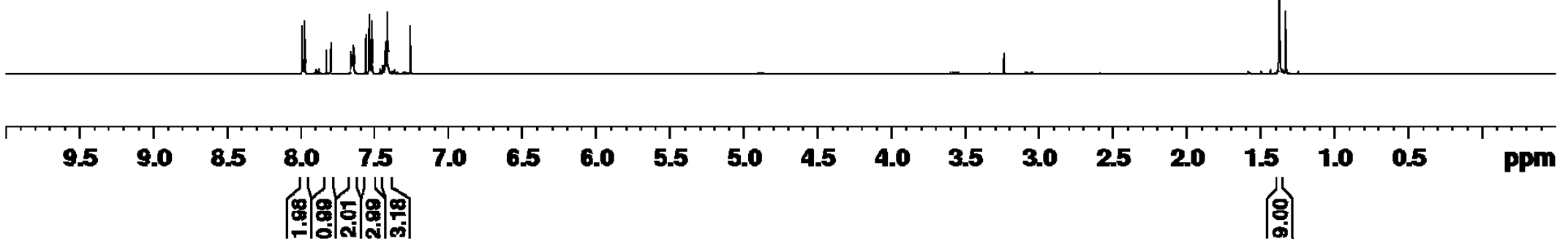
Supporting Information for Organometallic

S67

Figure S37. ${ }^{13} \mathrm{C}$ NMR (126 MHz, $\left.\mathrm{CDCl}_{3}\right)$ of 13.
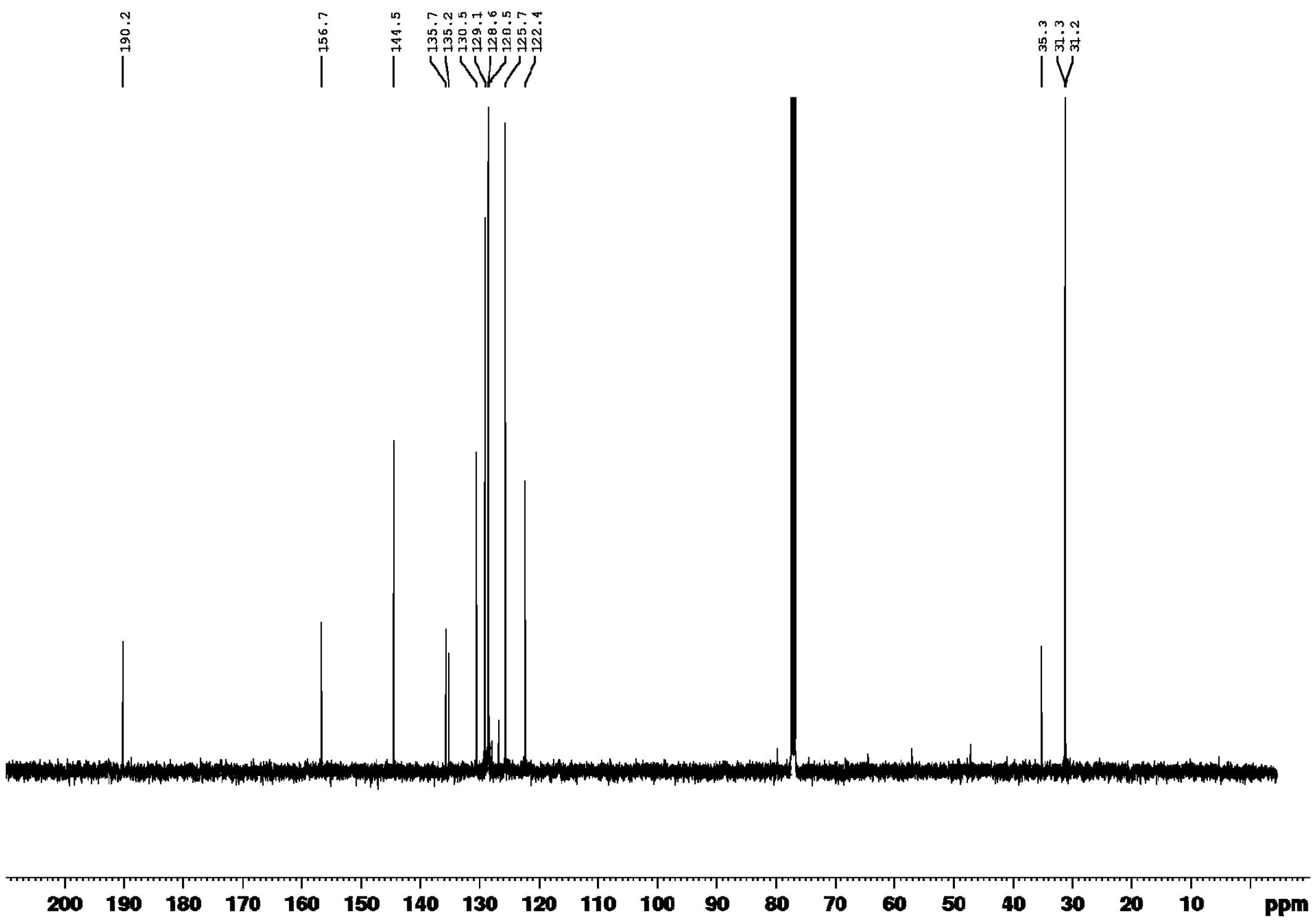
Figure S38. ${ }^{1} \mathrm{H} \mathrm{NMR}\left(500 \mathrm{MHz}, \mathrm{CDCl}_{3}\right)$ of 14.

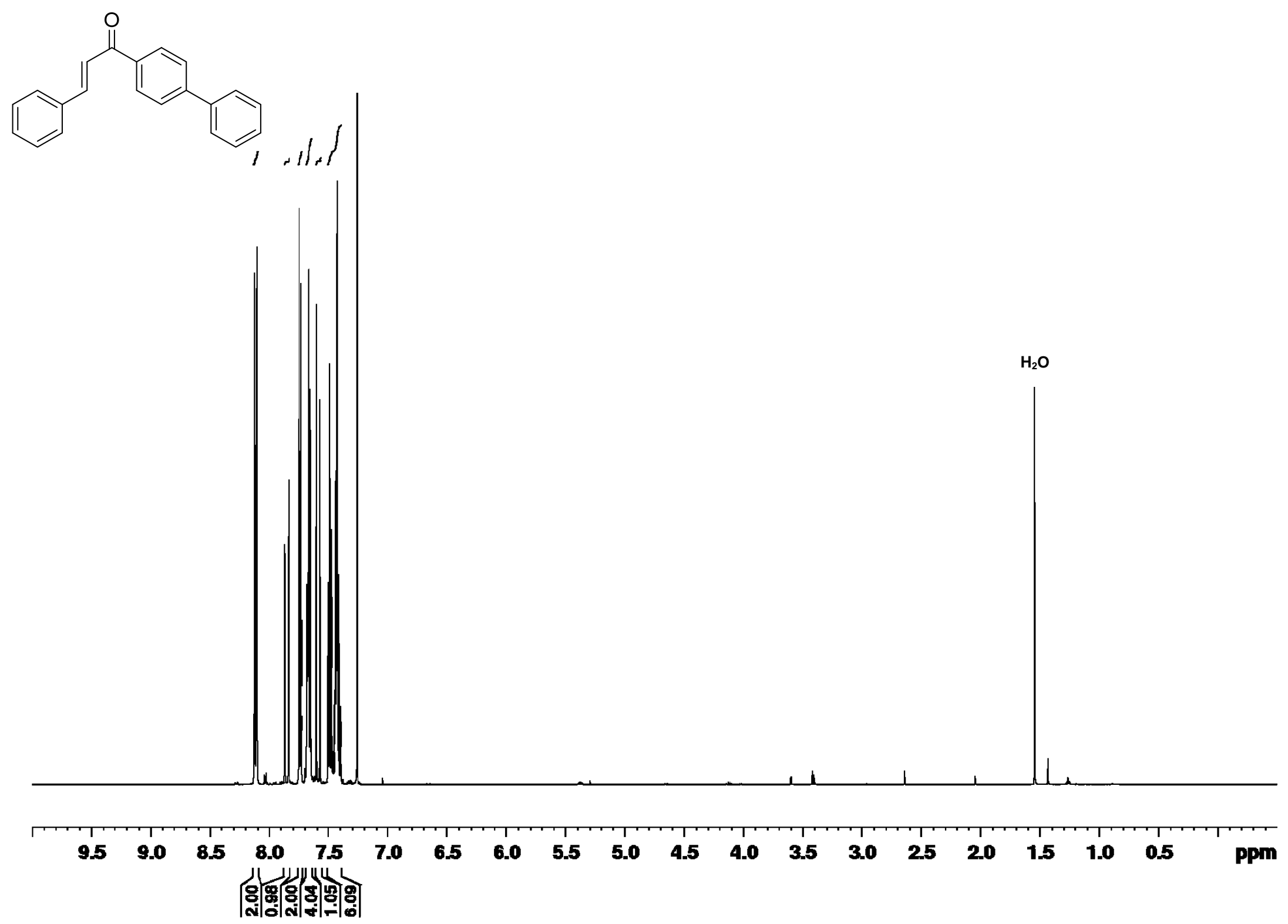


Supporting Information for Organometallic

S69

Figure S39. ${ }^{13} \mathrm{C}$ NMR (126 MHz, $\left.\mathrm{CDCl}_{3}\right)$ of 14.

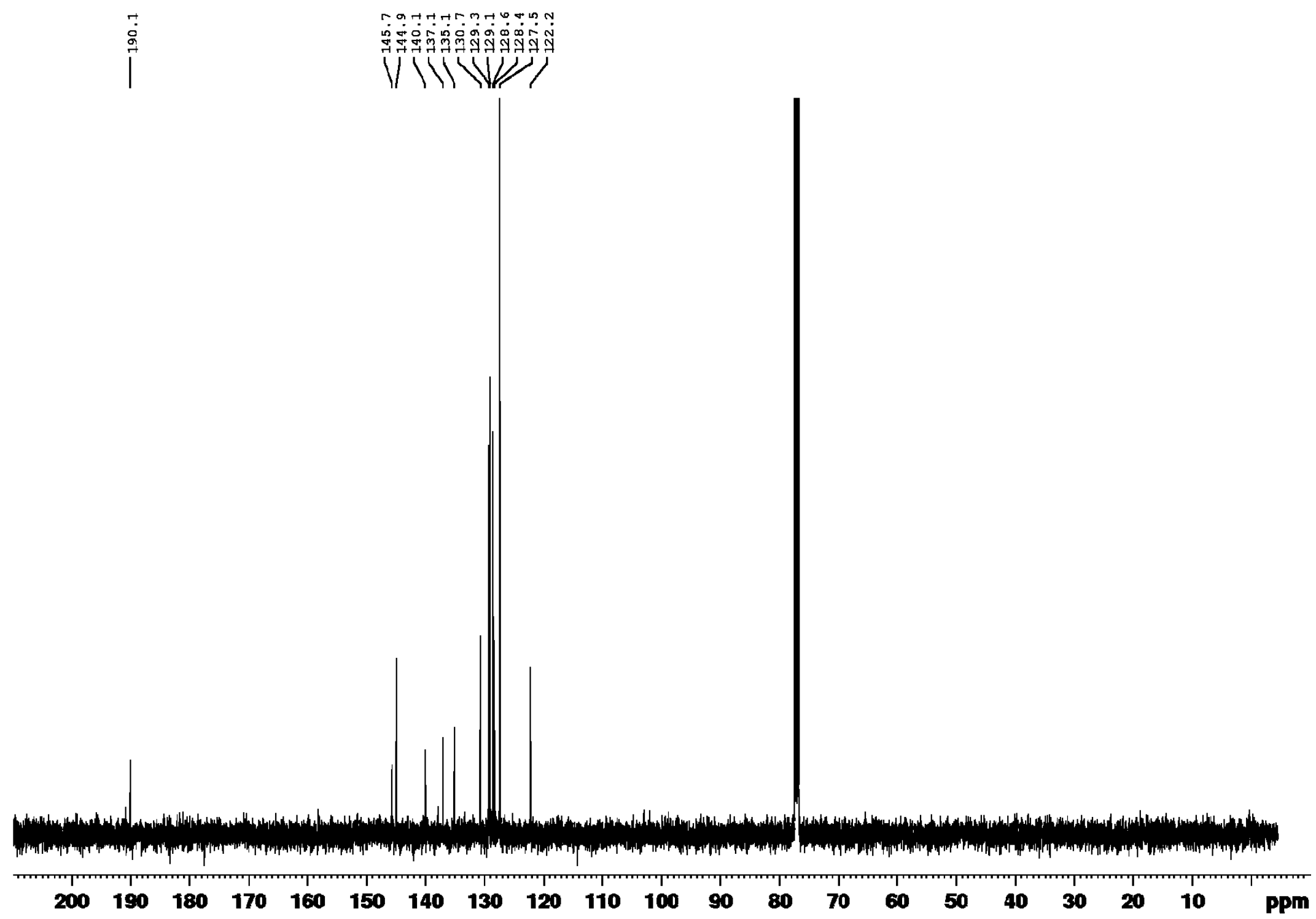


Supporting Information for Organometallic

$\mathrm{S} 70$

Figure S40. ${ }^{1} \mathrm{H} \mathrm{NMR}\left(500 \mathrm{MHz}, \mathrm{CDCl}_{3}\right)$ of 15.<smiles>Cc1ccccc1C(=O)/C=C/c1ccccc1</smiles>

1,16
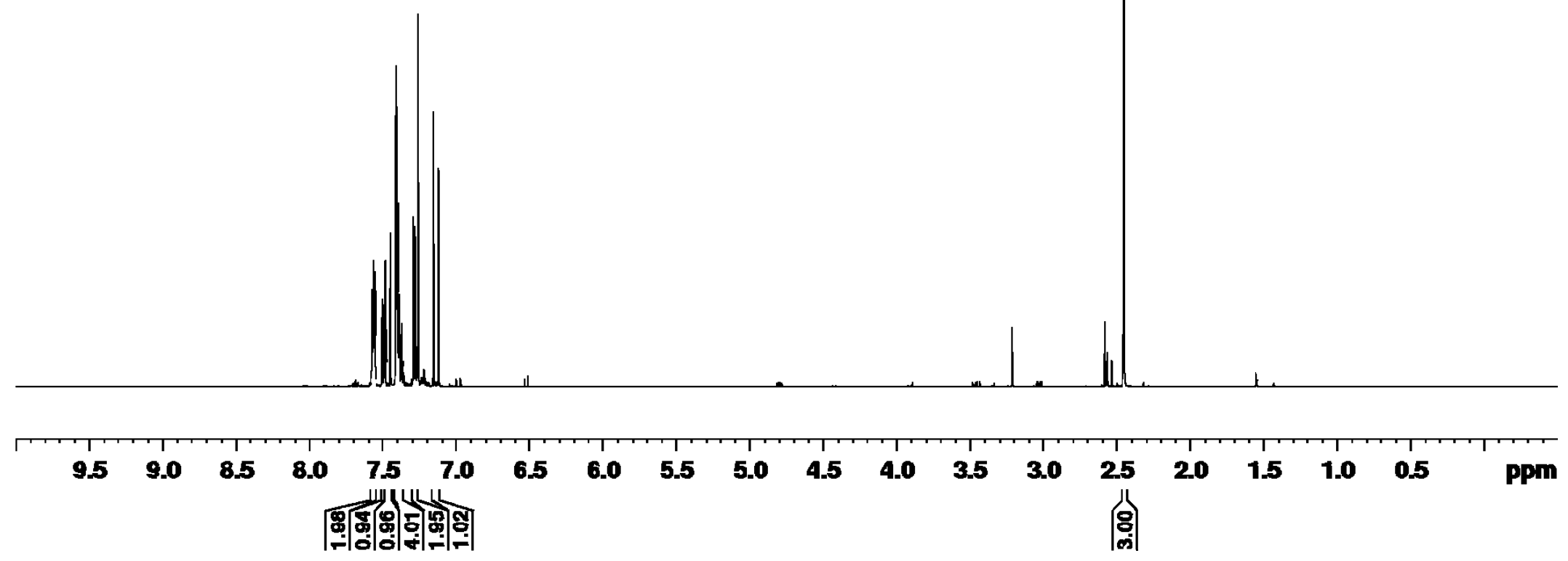
Figure S41. ${ }^{13} \mathrm{C}$ NMR (126 MHz, $\left.\mathrm{CDCl}_{3}\right)$ of 15.

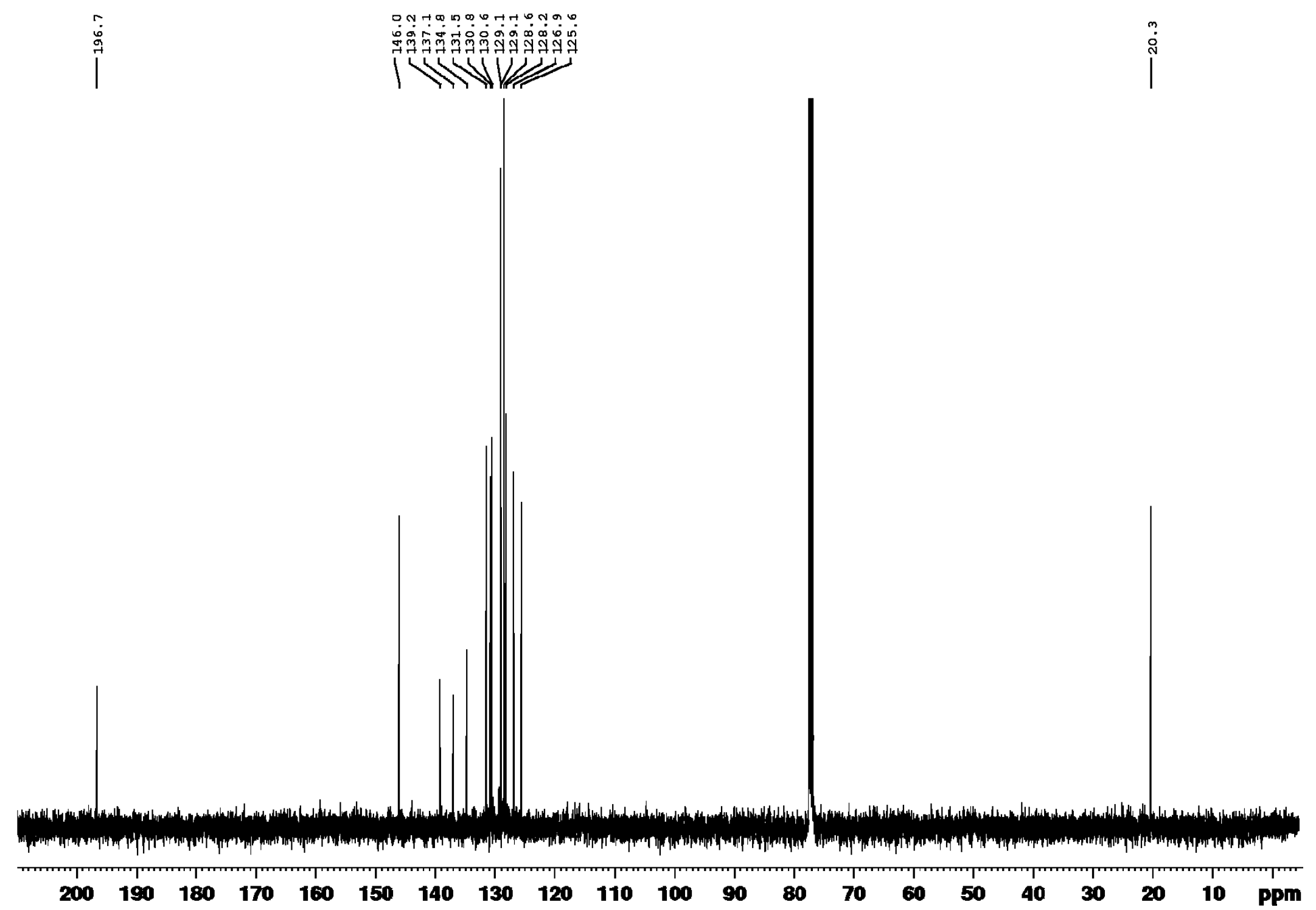




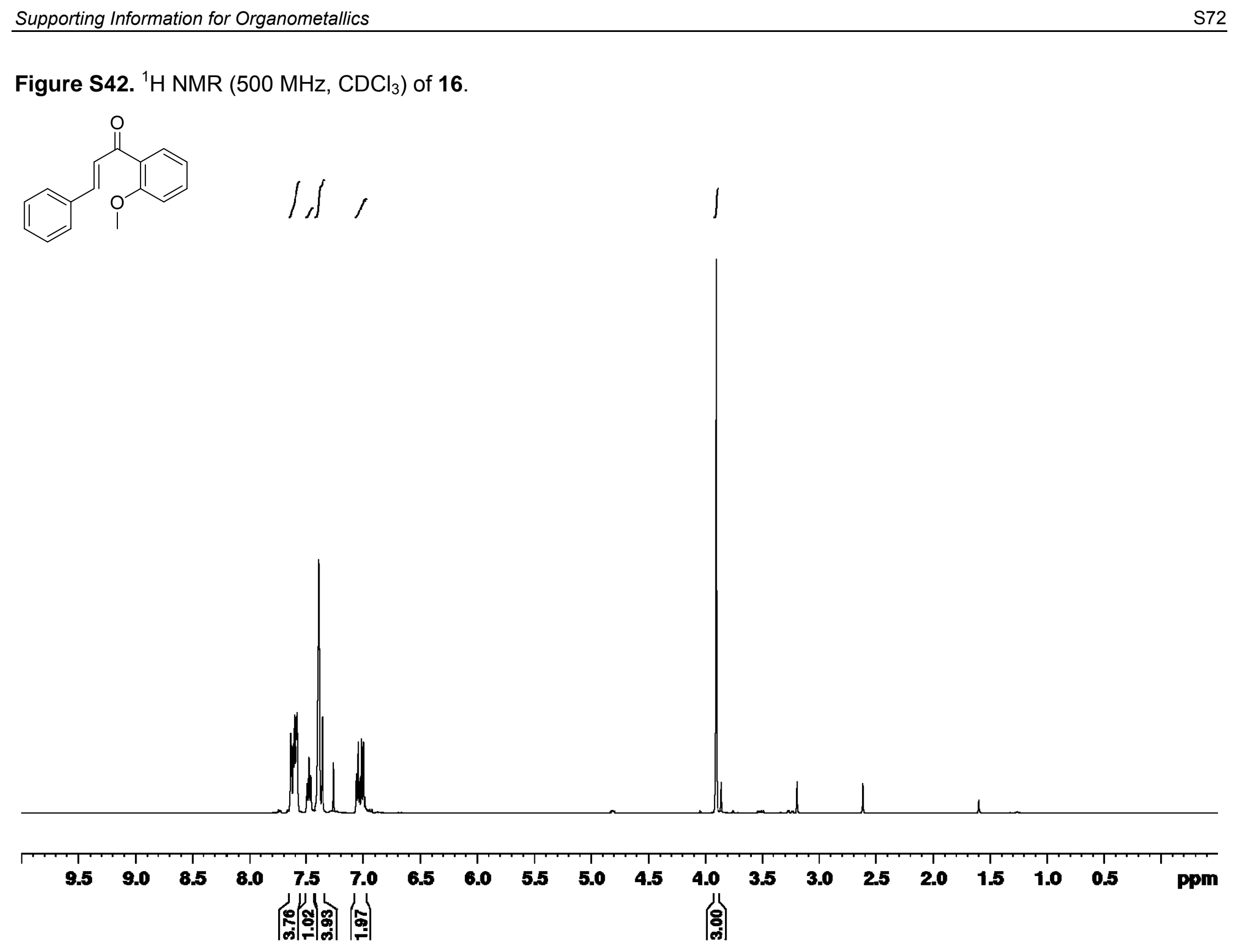


Supporting Information for Organometallic

S73

Figure S43. ${ }^{13} \mathrm{C}$ NMR (126 MHz, $\left.\mathrm{CDCl}_{3}\right)$ of 16.

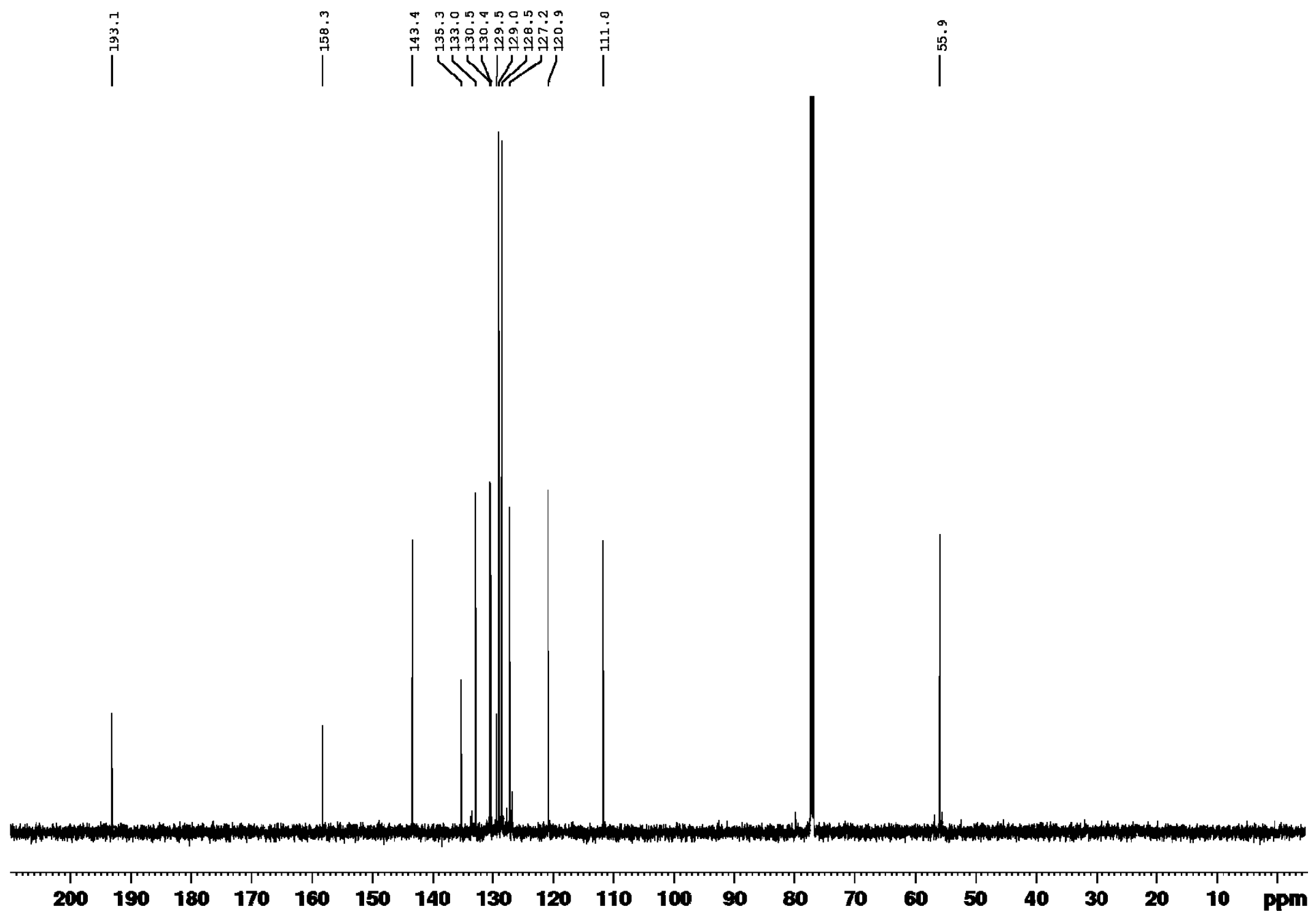


Supporting Information for Organometallic

S74

Figure S44. ${ }^{1} \mathrm{H}$ NMR $\left(500 \mathrm{MHz}, \mathrm{CDCl}_{3}\right)$ of 17.
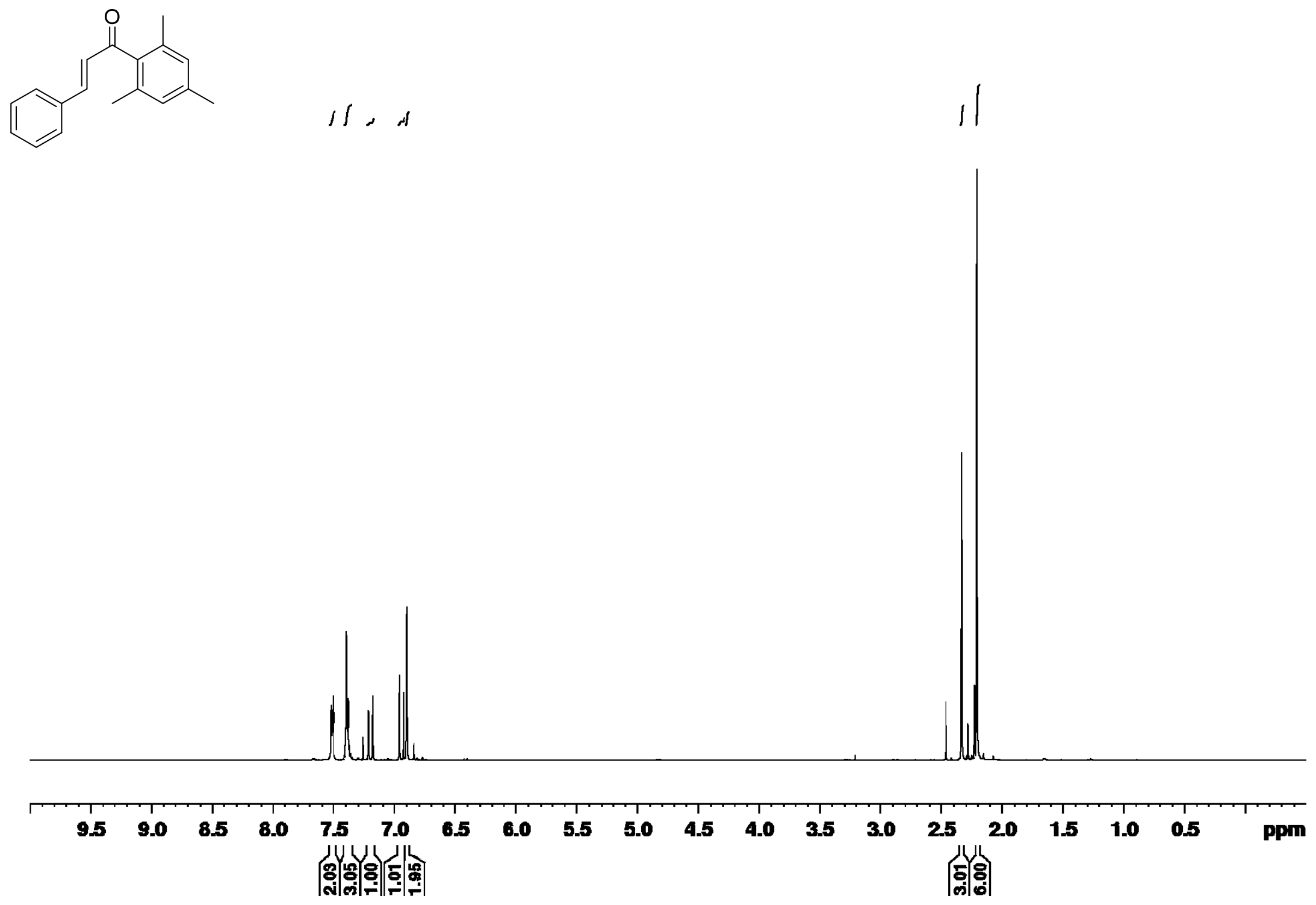
Figure S45. ${ }^{13} \mathrm{C}$ NMR $\left(126 \mathrm{MHz}, \mathrm{CDCl}_{3}\right)$ of 17.

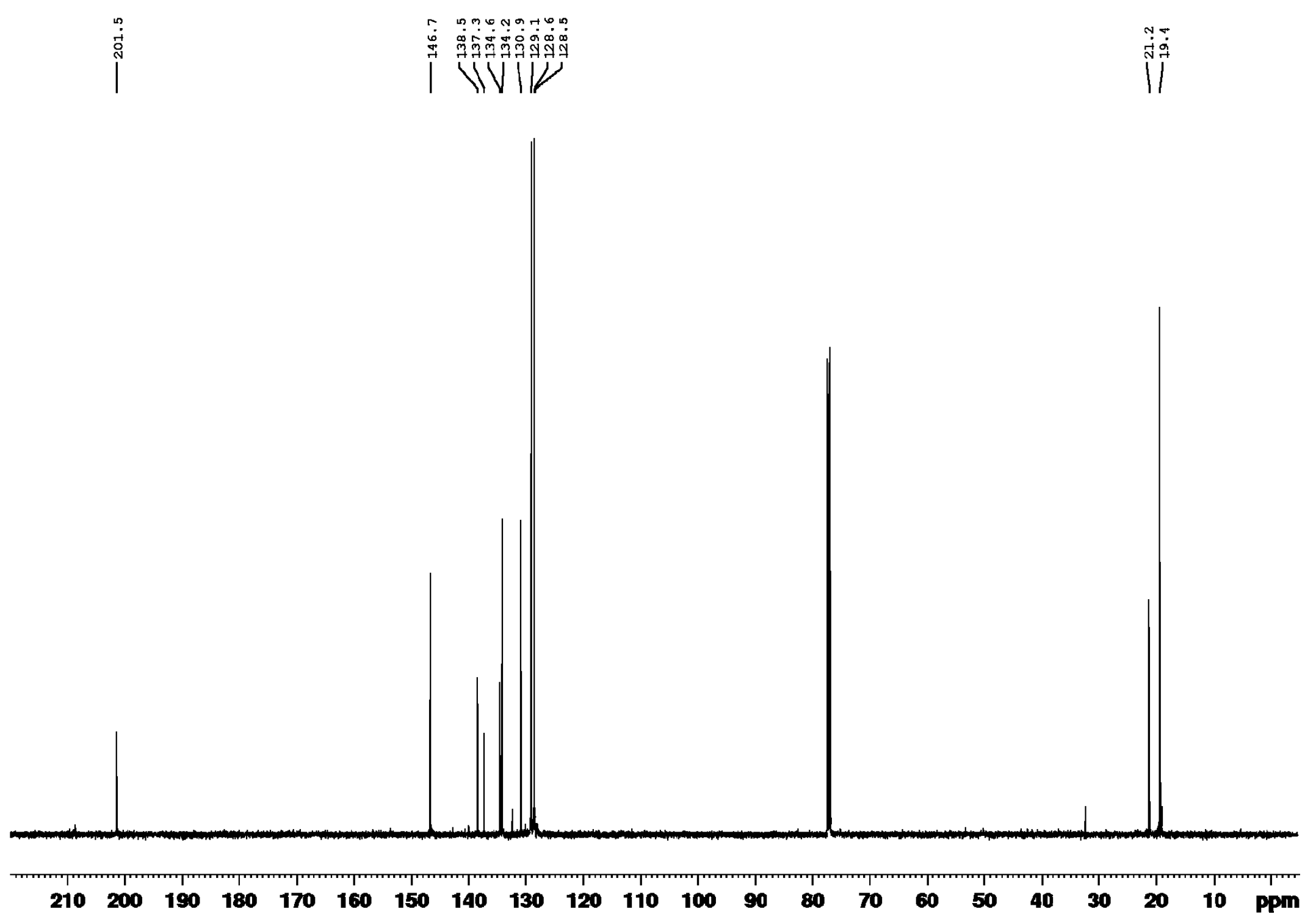


Figure S46. ${ }^{1} \mathrm{H} \mathrm{NMR}\left(500 \mathrm{MHz}, \mathrm{CDCl}_{3}\right)$ of 18.

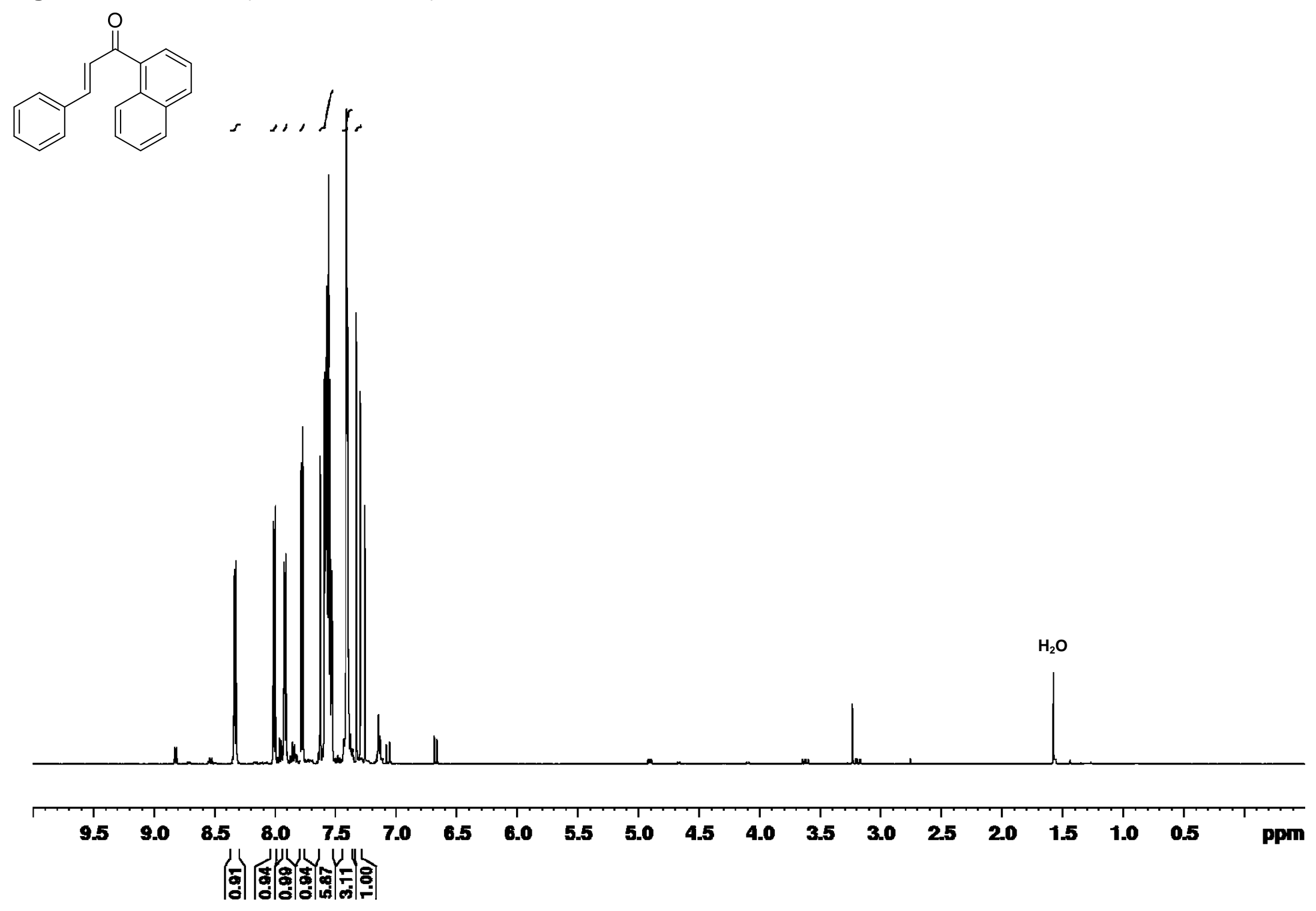


Figure S47. ${ }^{13} \mathrm{C} \mathrm{NMR}\left(126 \mathrm{MHz}, \mathrm{CDCl}_{3}\right)$ of 18.

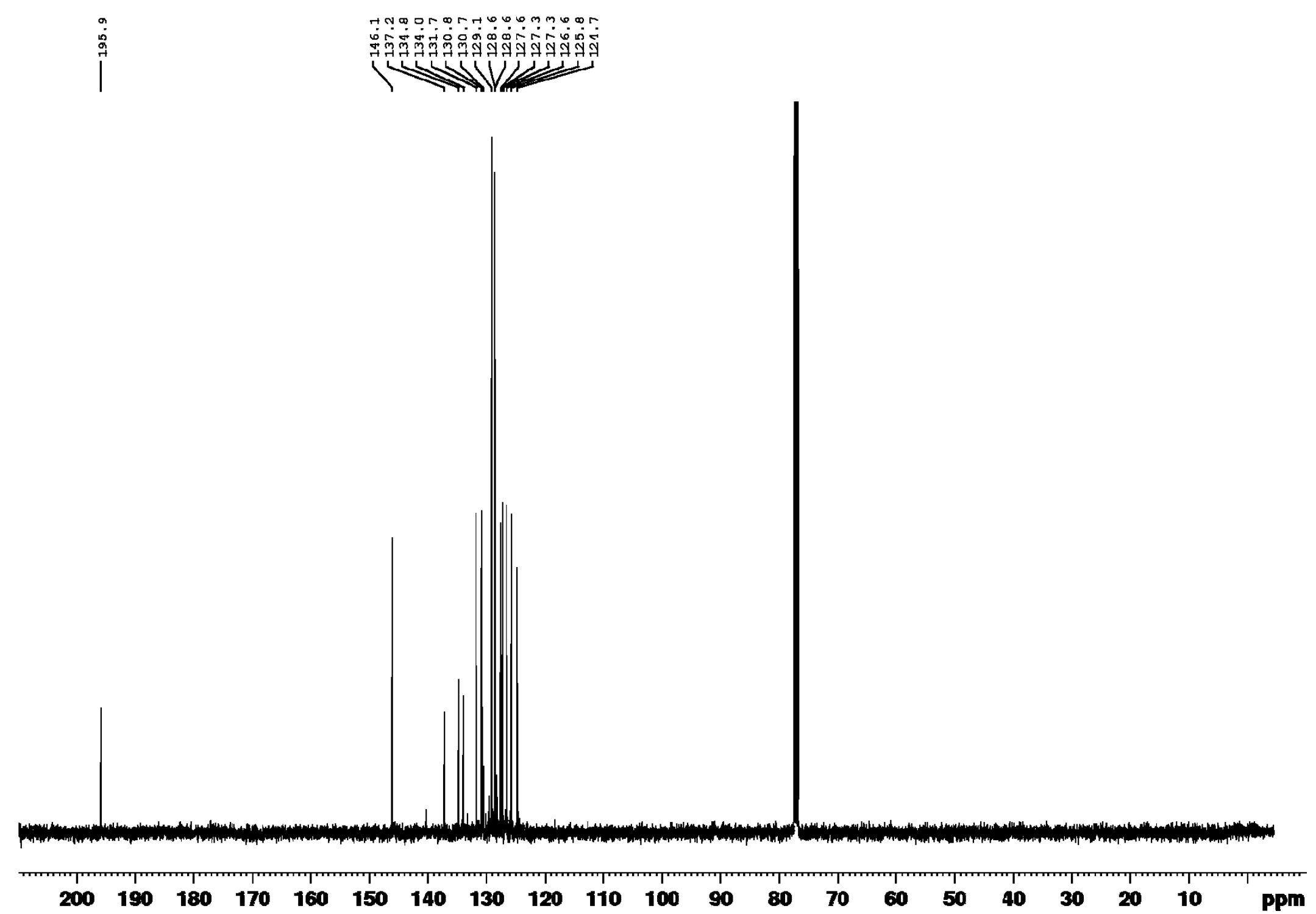


Figure S48. ${ }^{1} \mathrm{H} \mathrm{NMR}\left(500 \mathrm{MHz}, \mathrm{CDCl}_{3}\right)$ of 19.

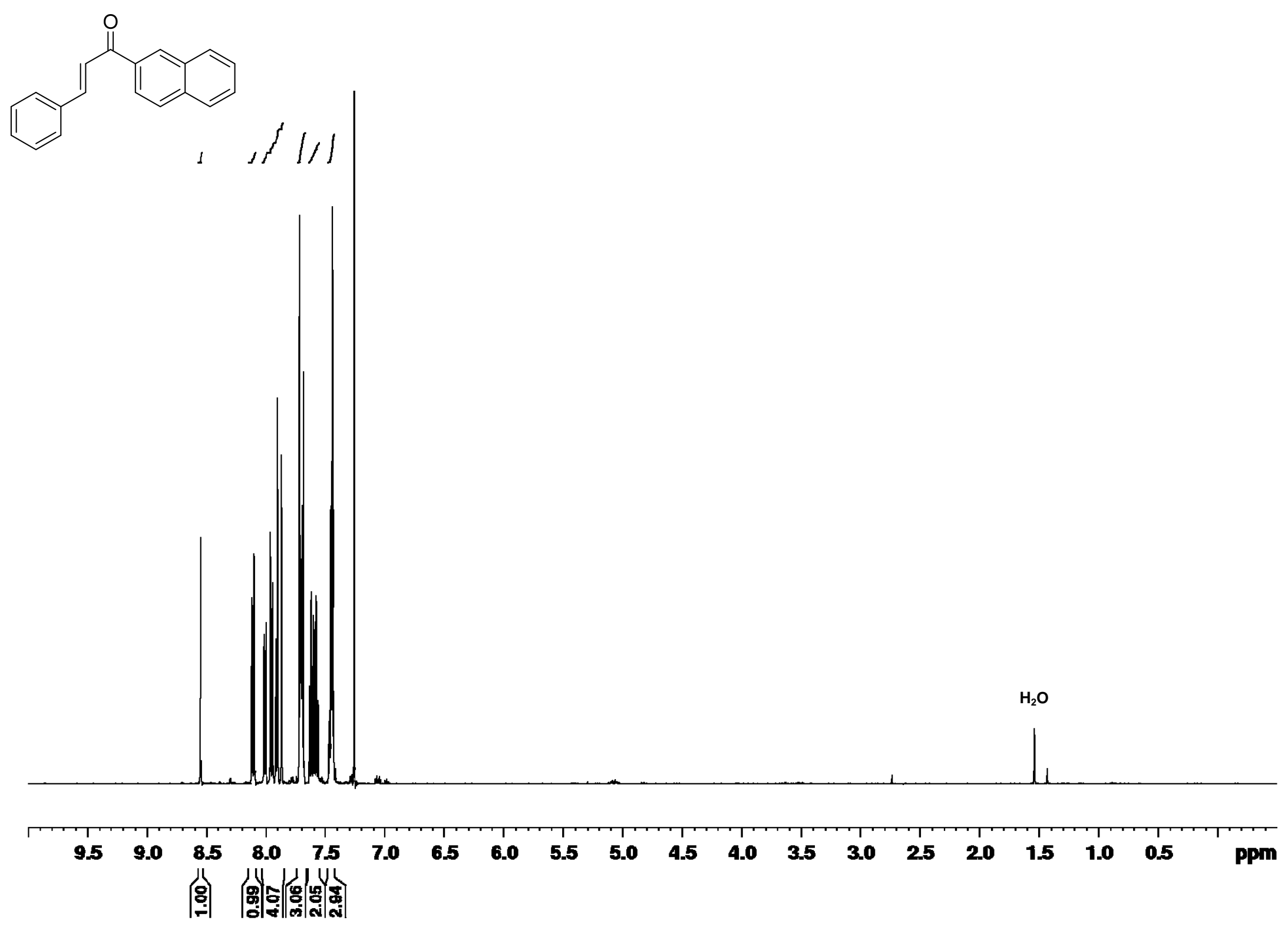


Supporting Information for Organometallic

S79

Figure S49. ${ }^{13} \mathrm{C}$ NMR $\left(126 \mathrm{MHz}, \mathrm{CDCl}_{3}\right)$ of 19.

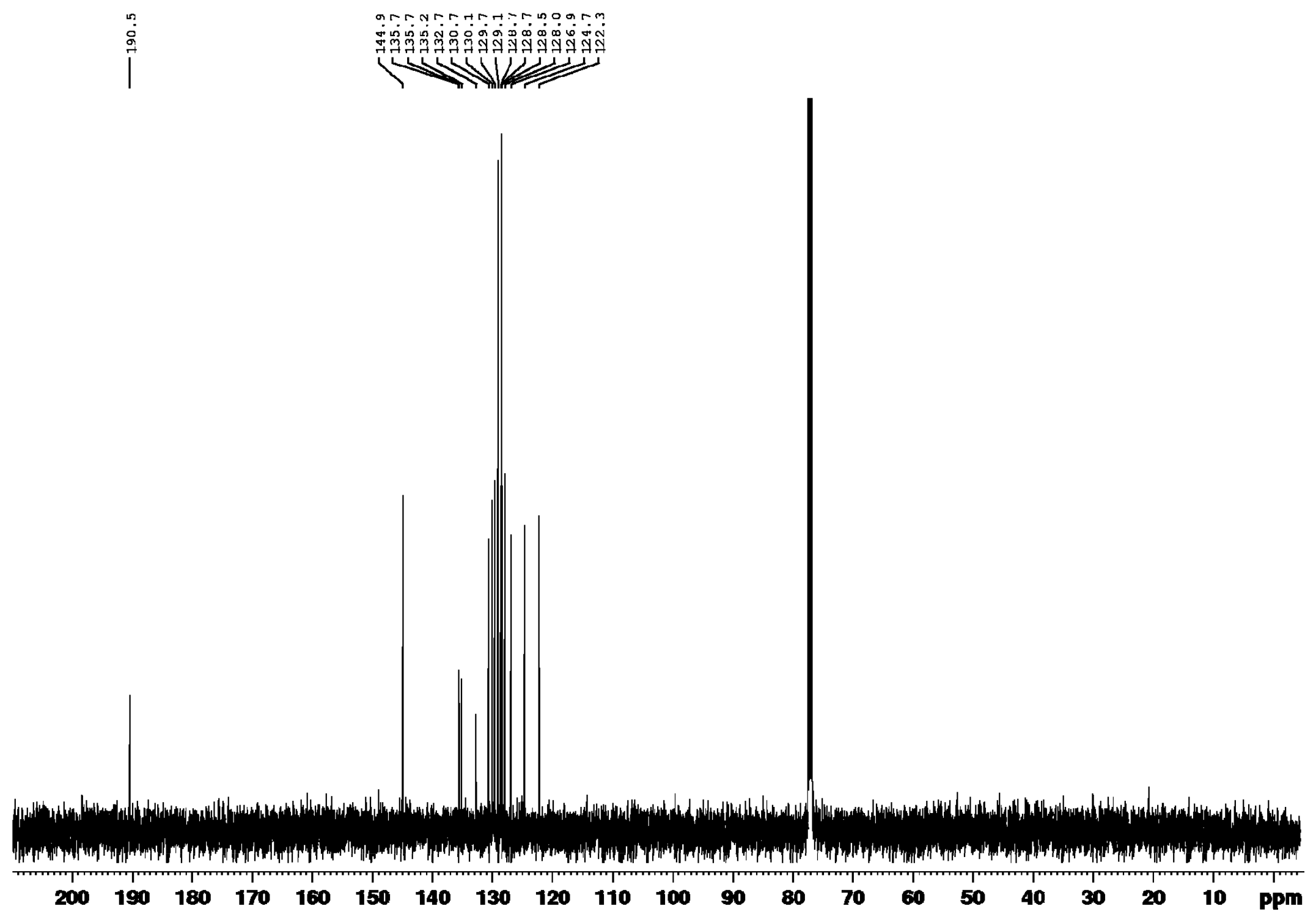


Figure S50. ${ }^{1} \mathrm{H}$ NMR $\left(500 \mathrm{MHz}, \mathrm{CDCl}_{3}\right)$ of 20.

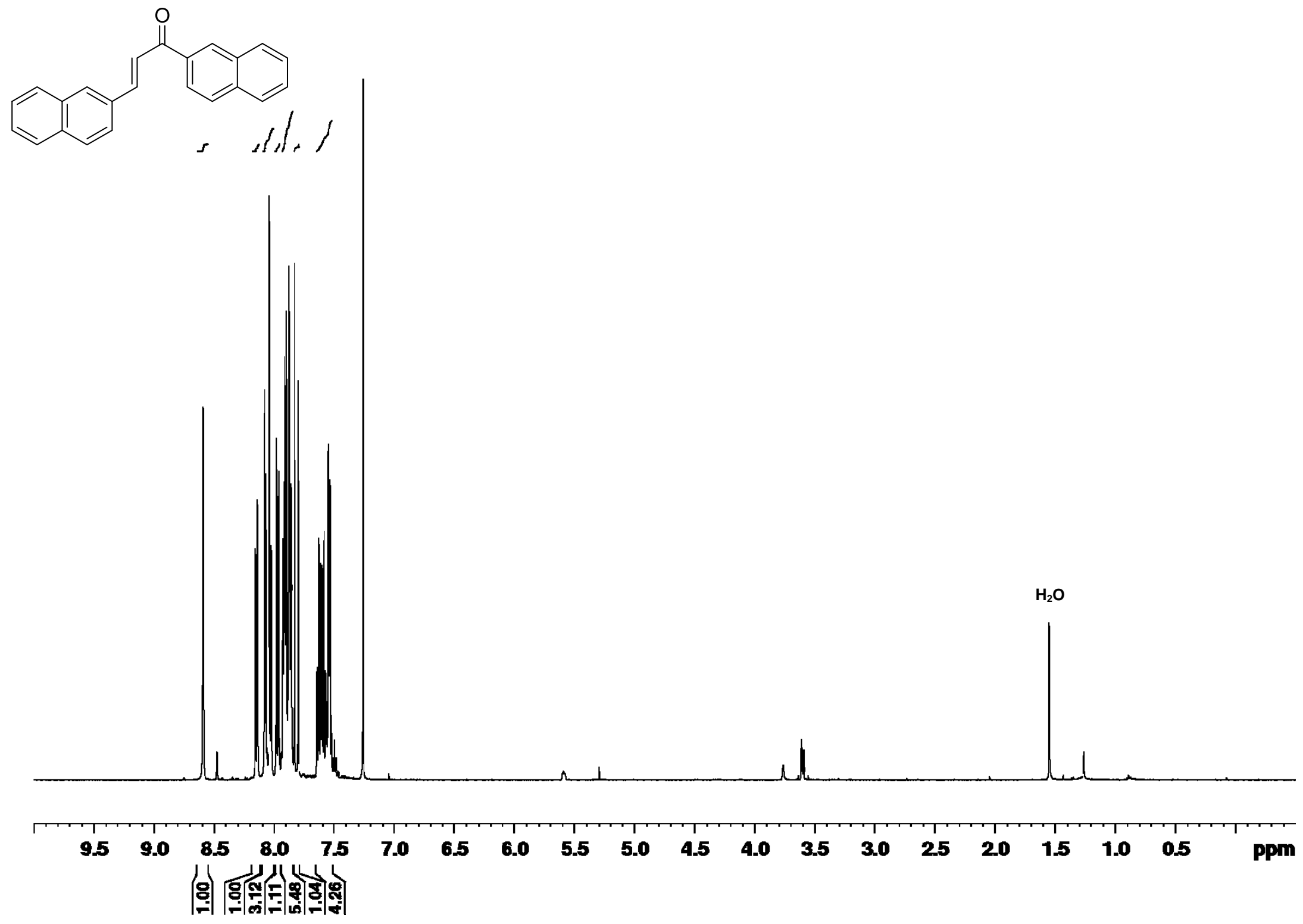


Supporting Information for Organometallic

S81

Figure S51. ${ }^{13} \mathrm{C}$ NMR (126 MHz, $\left.\mathrm{CDCl}_{3}\right)$ of 20.

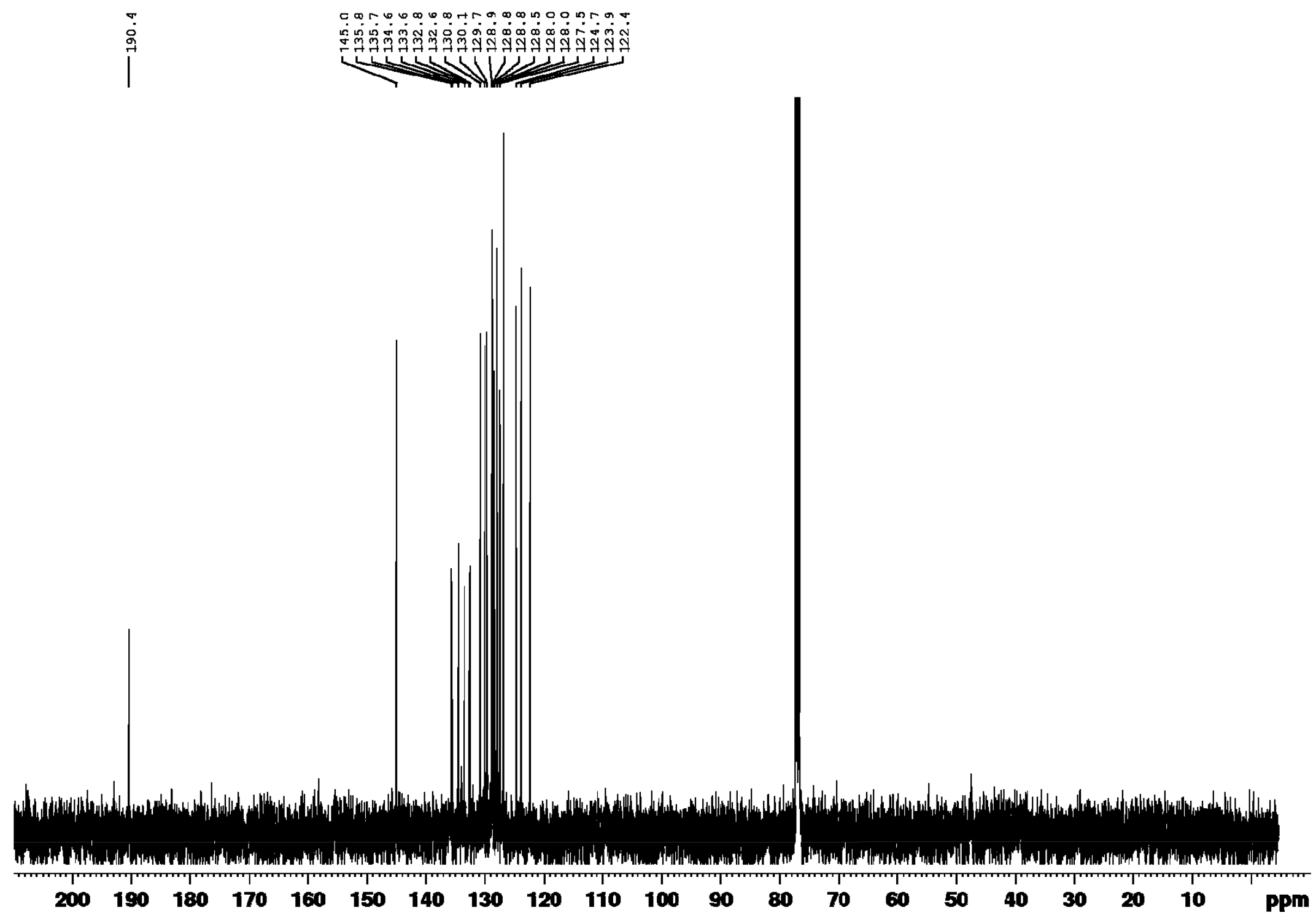


Supporting Information for Organometallic

S82

Figure S52. ${ }^{1} \mathrm{H}$ NMR $\left(500 \mathrm{MHz}, \mathrm{CDCl}_{3}\right)$ of $\mathbf{3}$.
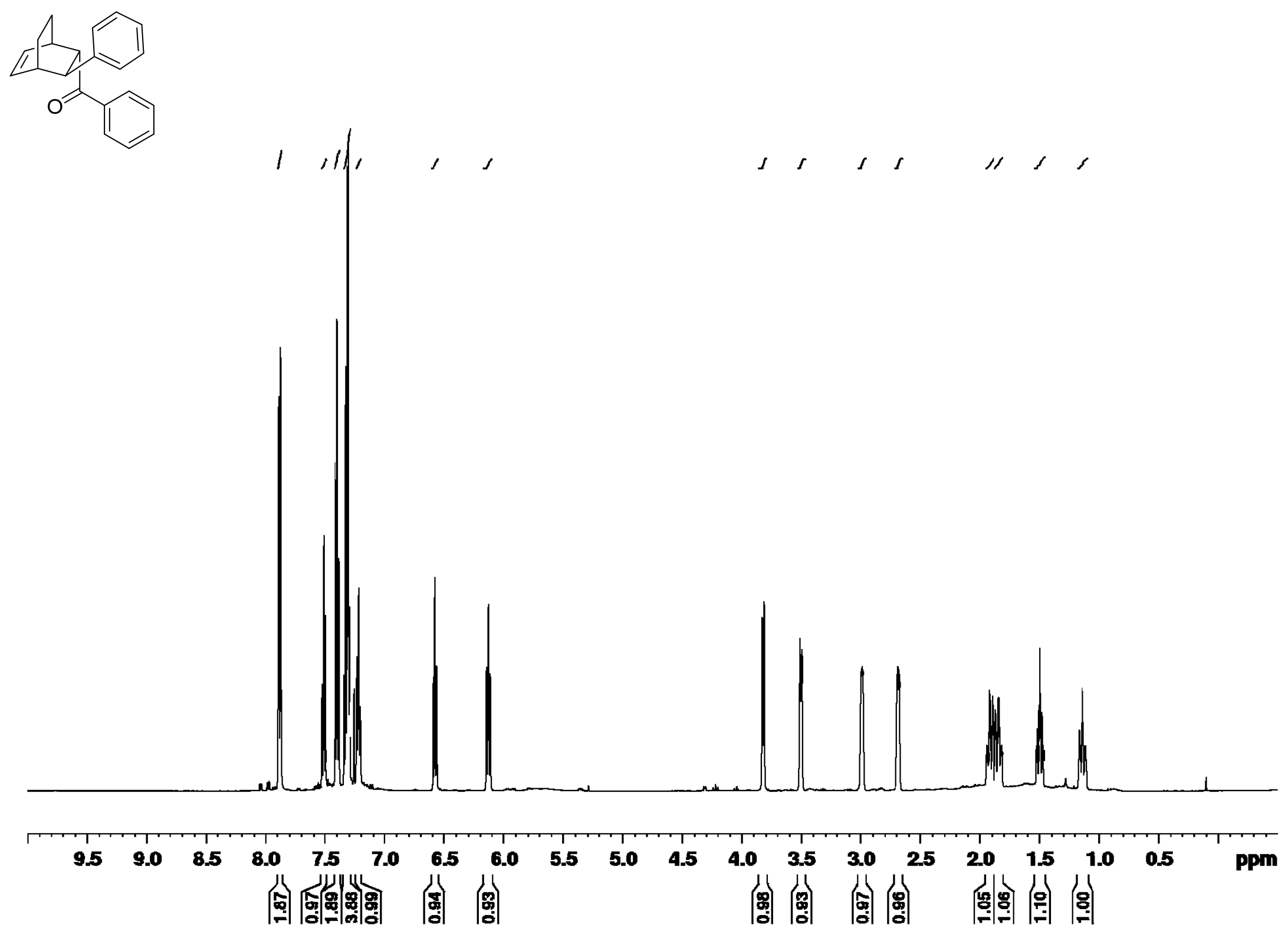
Figure S53. ${ }^{13} \mathrm{C}$ NMR $\left(126 \mathrm{MHz}, \mathrm{CDCl}_{3}\right)$ of $\mathbf{3}$.

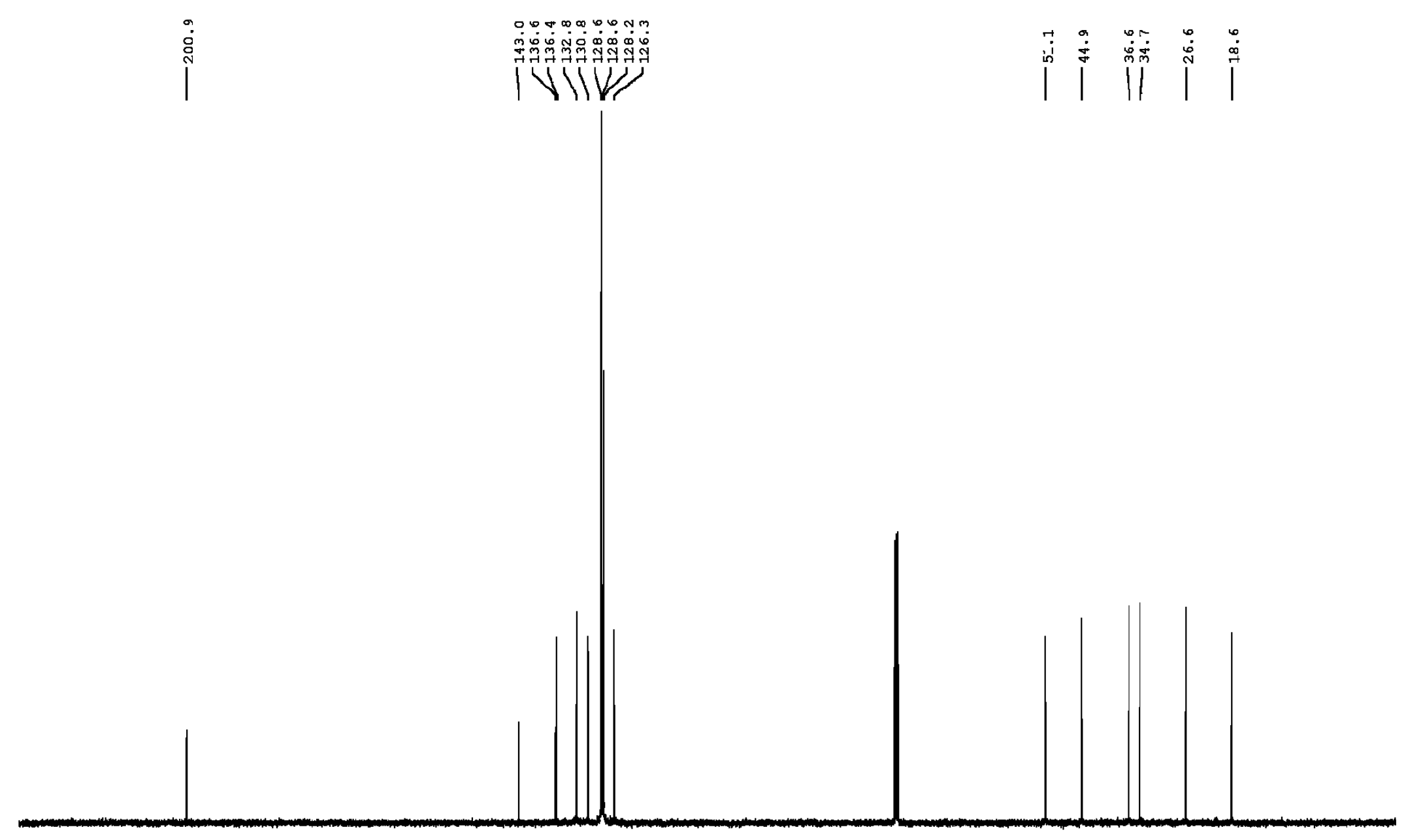

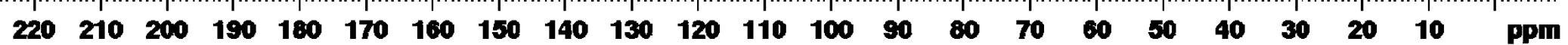


Supporting Information for Organometallics

S84

Figure S54. ${ }^{1} \mathrm{H}$ NMR (500 MHz, CDCl $)$ of 21.
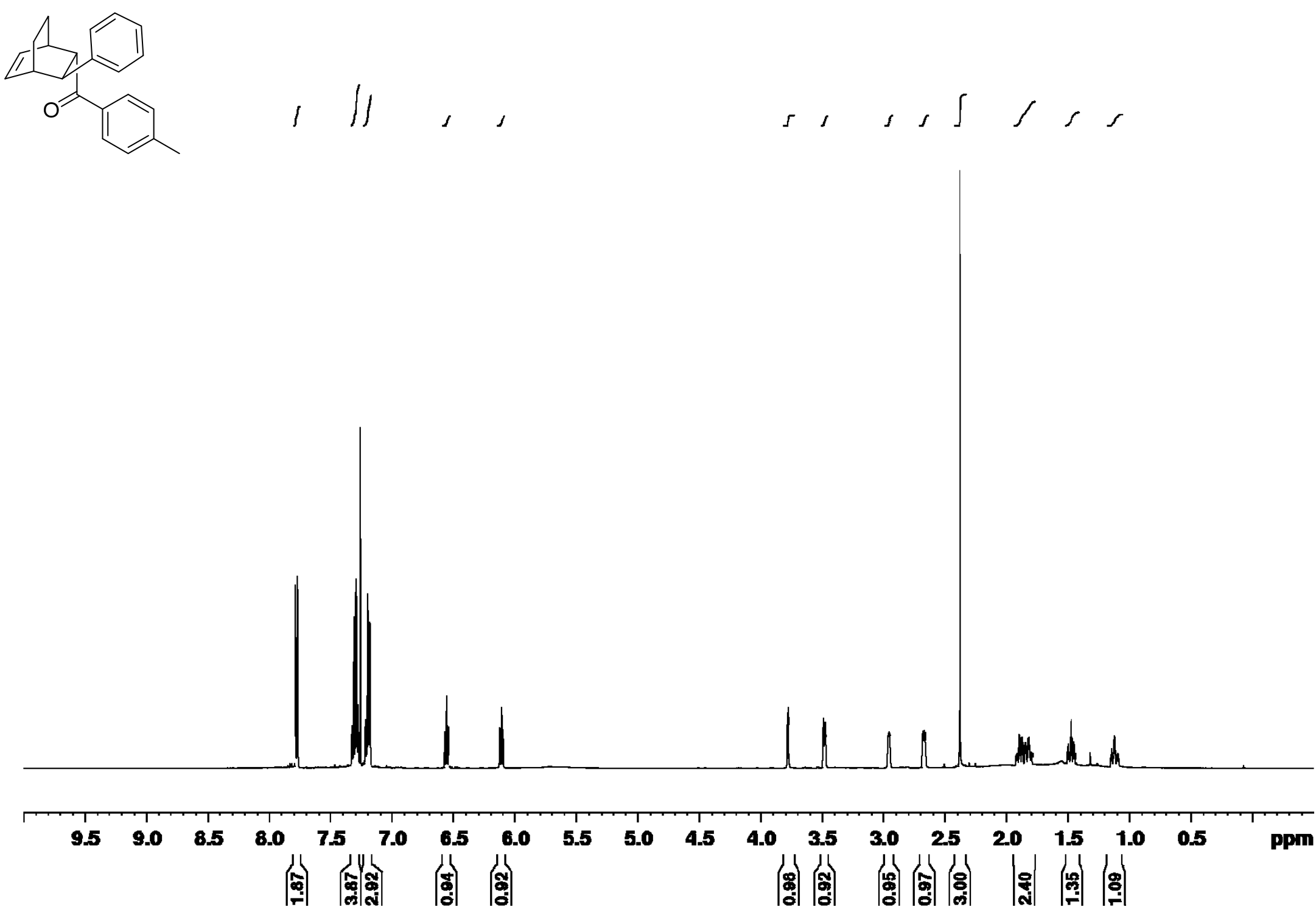
Figure S55. ${ }^{13} \mathrm{C}$ NMR $\left(126 \mathrm{MHz}, \mathrm{CDCl}_{3}\right)$ of 21.

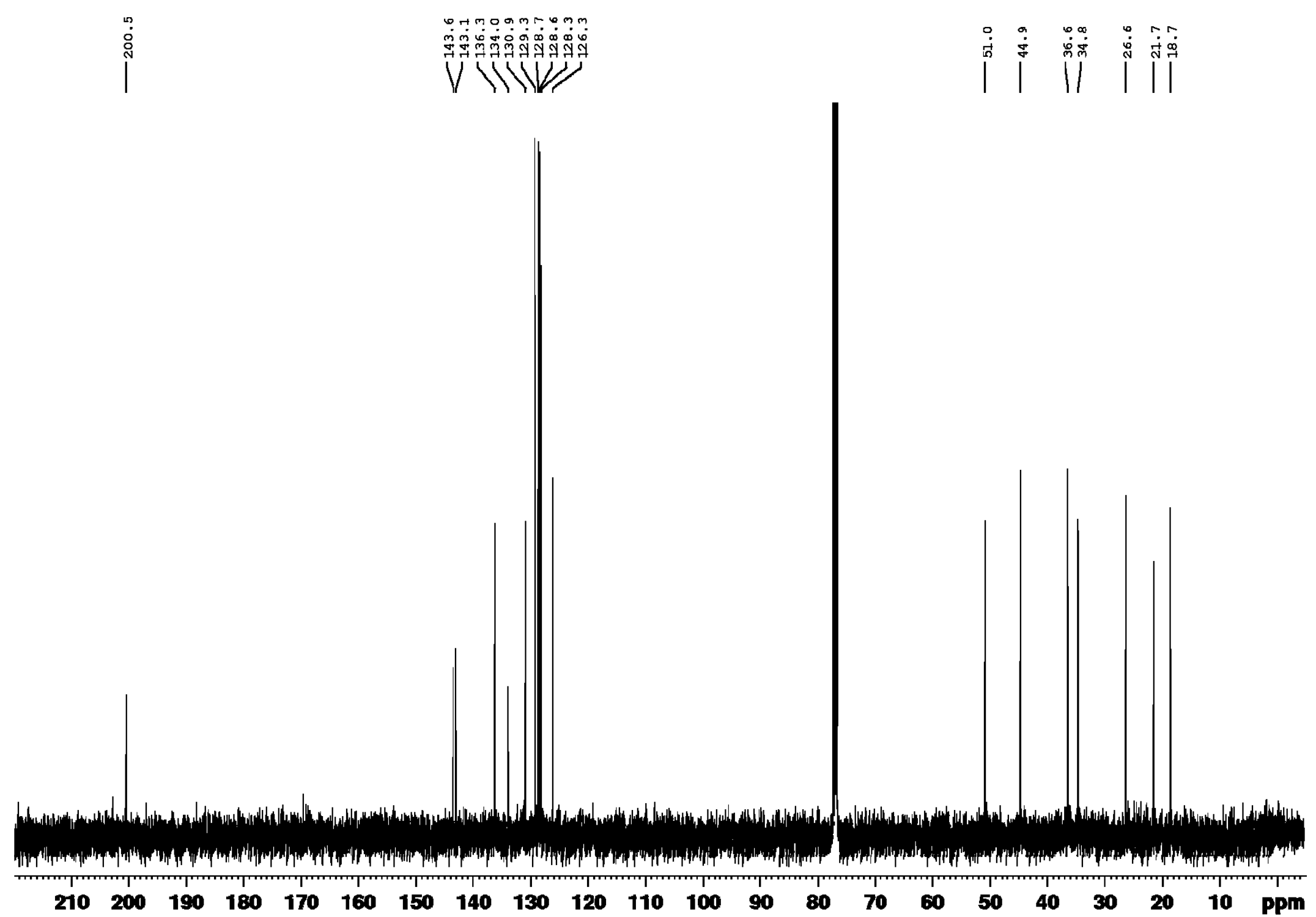


Figure S56. ${ }^{1} \mathrm{H} \mathrm{NMR}\left(400 \mathrm{MHz}, \mathrm{CDCl}_{3}\right)$ of 22.
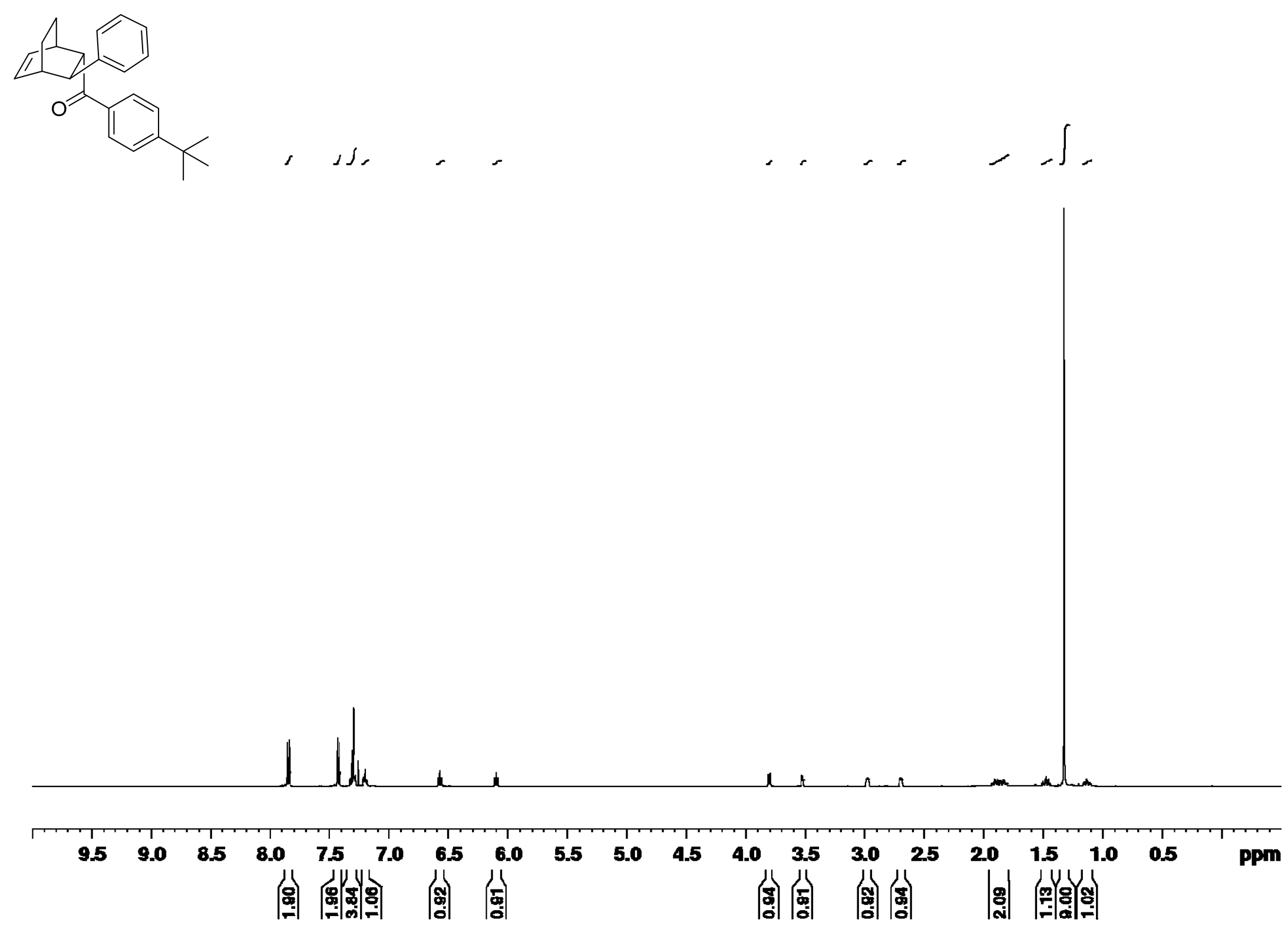
Figure S57. ${ }^{13} \mathrm{C} \mathrm{NMR}\left(126 \mathrm{MHz}, \mathrm{CDCl}_{3}\right)$ of 22.

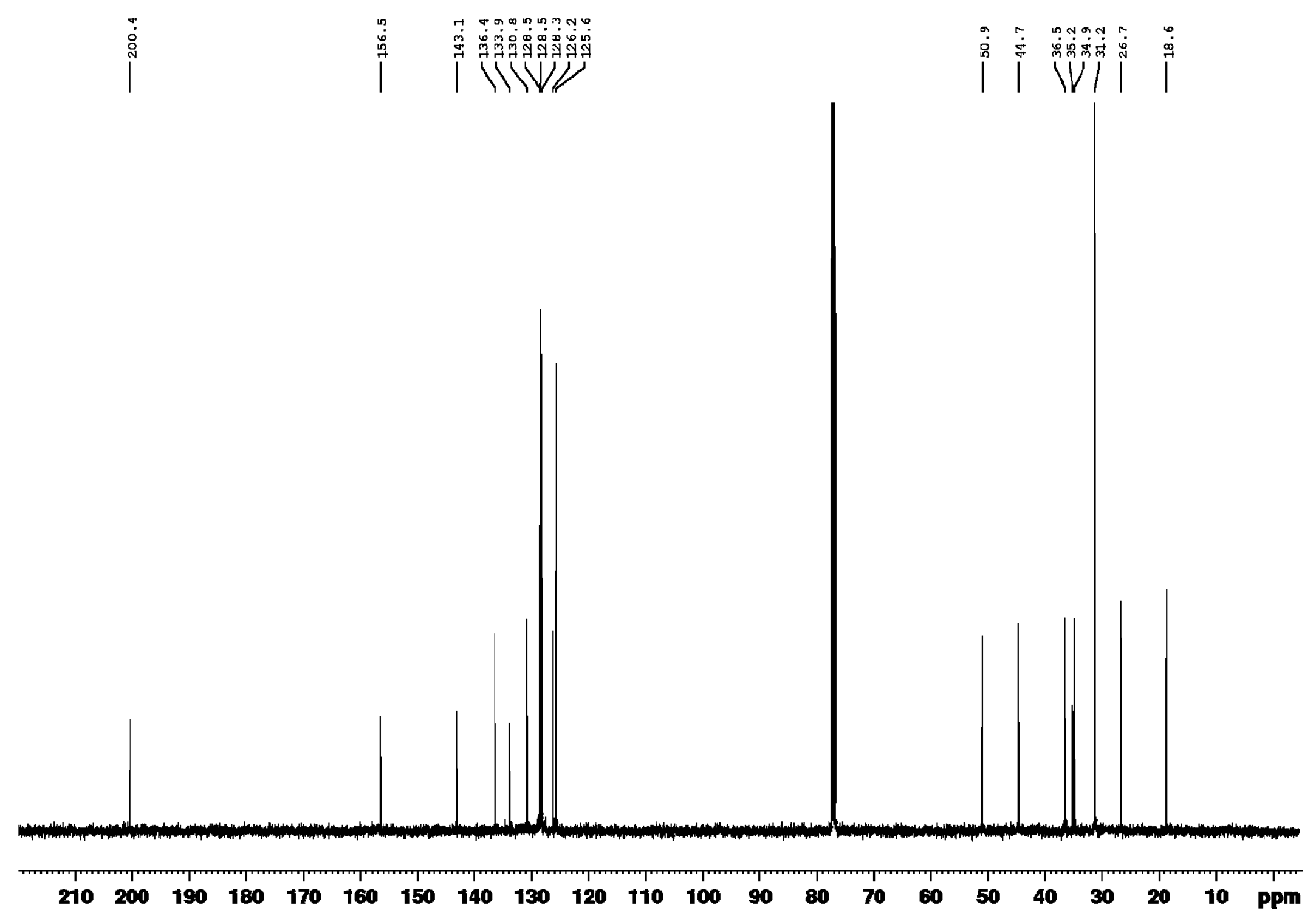




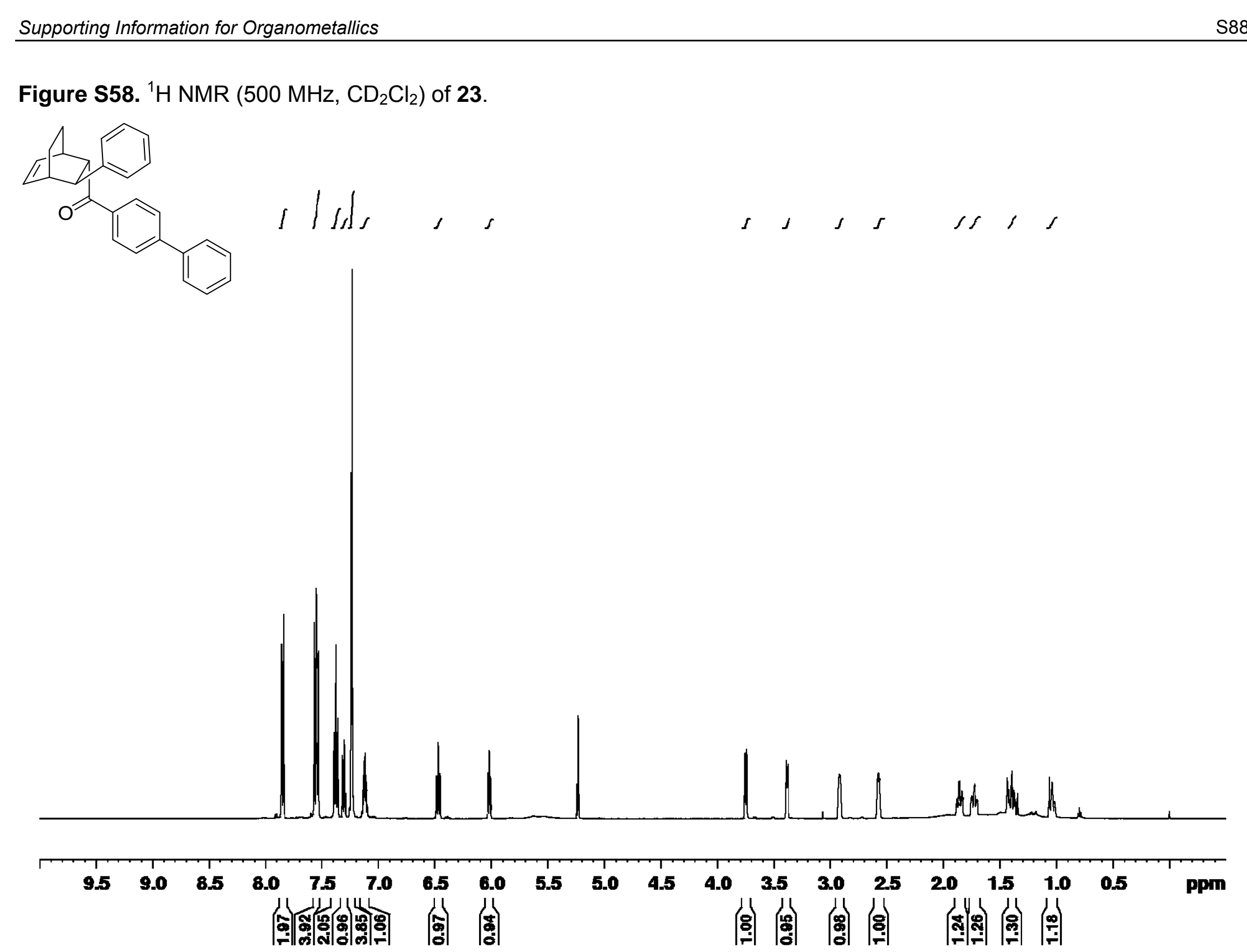


Figure S59. ${ }^{13} \mathrm{C}$ NMR $\left(126 \mathrm{MHz}, \mathrm{CD}_{2} \mathrm{Cl}_{2}\right)$ of 23.

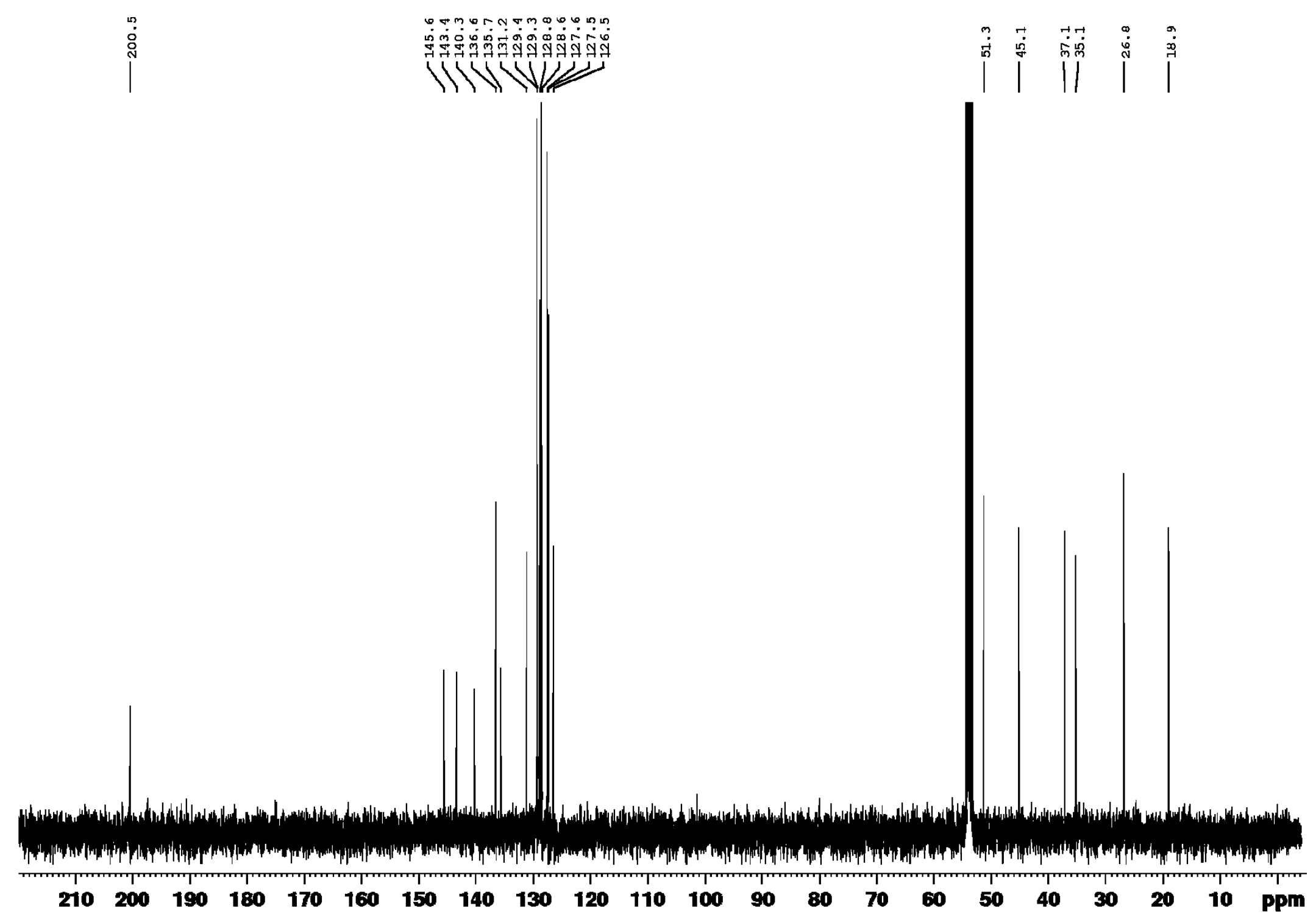


Supporting Information for Organometallic

S90

Figure S60. ${ }^{1} \mathrm{H}$ NMR $\left(500 \mathrm{MHz}, \mathrm{CDCl}_{3}\right)$ of 24.
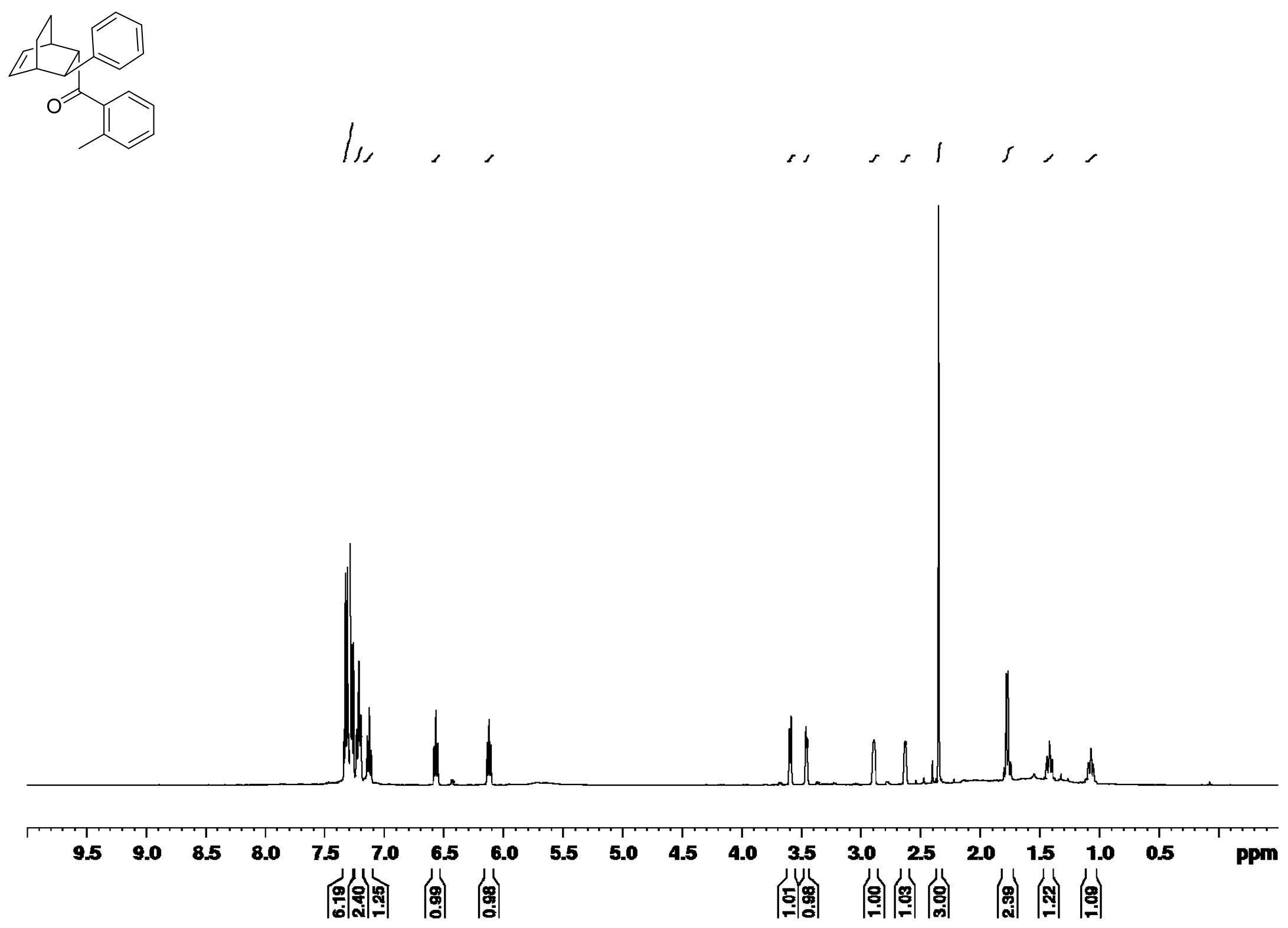
Figure S61. ${ }^{13} \mathrm{C}$ NMR (126 MHz, $\left.\mathrm{CDCl}_{3}\right)$ of 24.

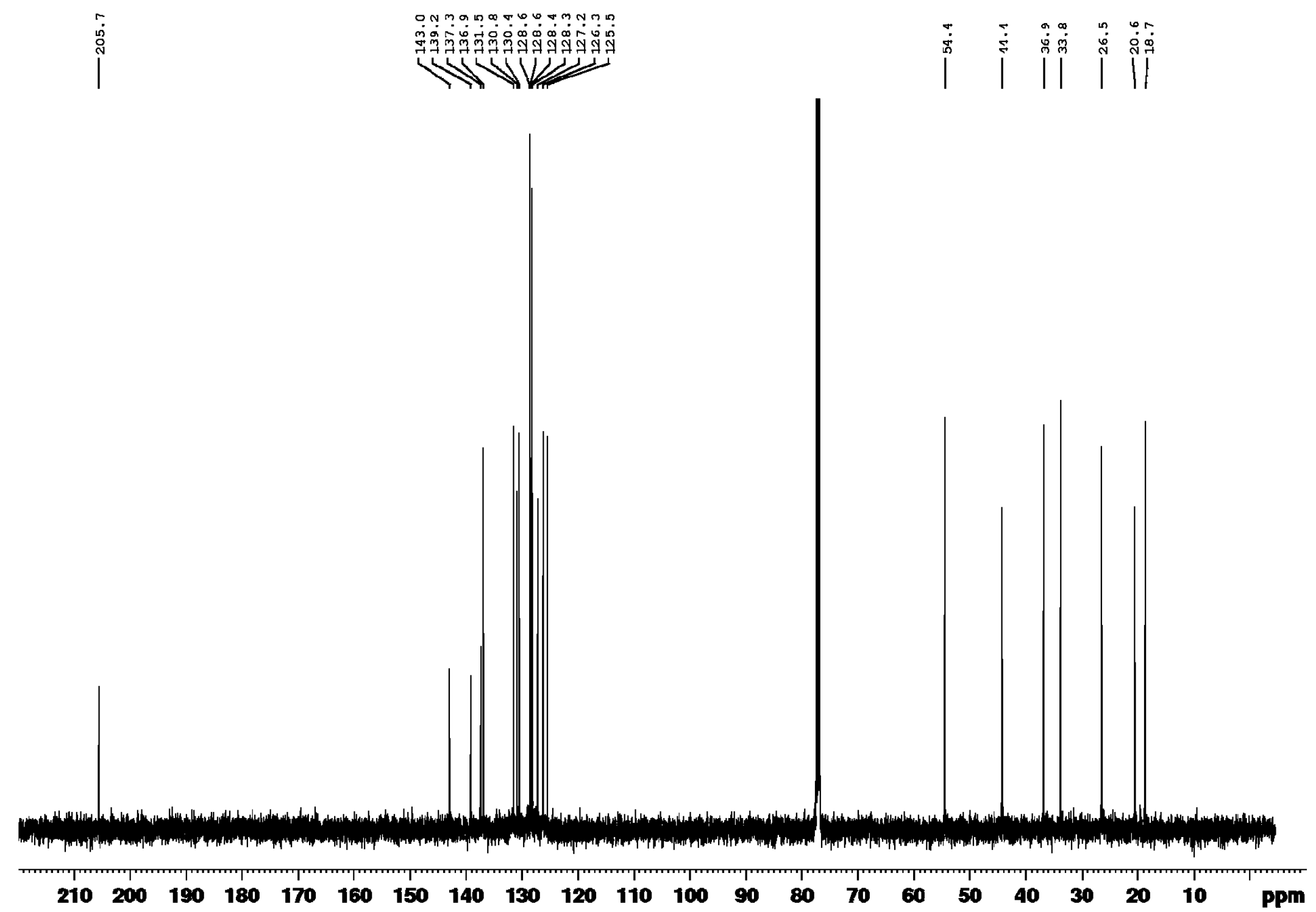


Figure S62. ${ }^{1} \mathrm{H} \mathrm{NMR}\left(500 \mathrm{MHz}, \mathrm{CDCl}_{3}\right)$ of 25.
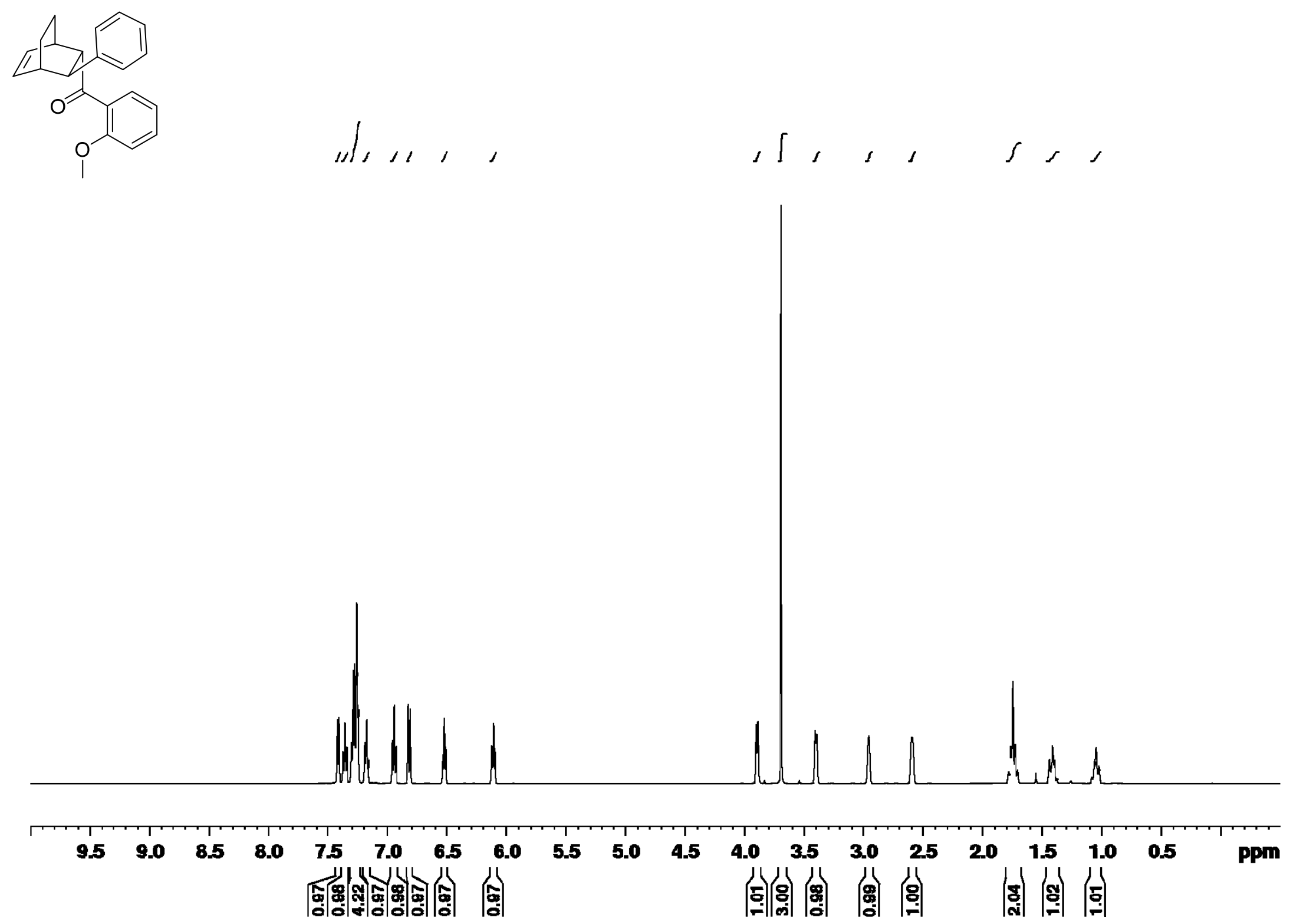
Figure S63. ${ }^{13} \mathrm{C}$ NMR $\left(126 \mathrm{MHz}, \mathrm{CDCl}_{3}\right)$ of 25.

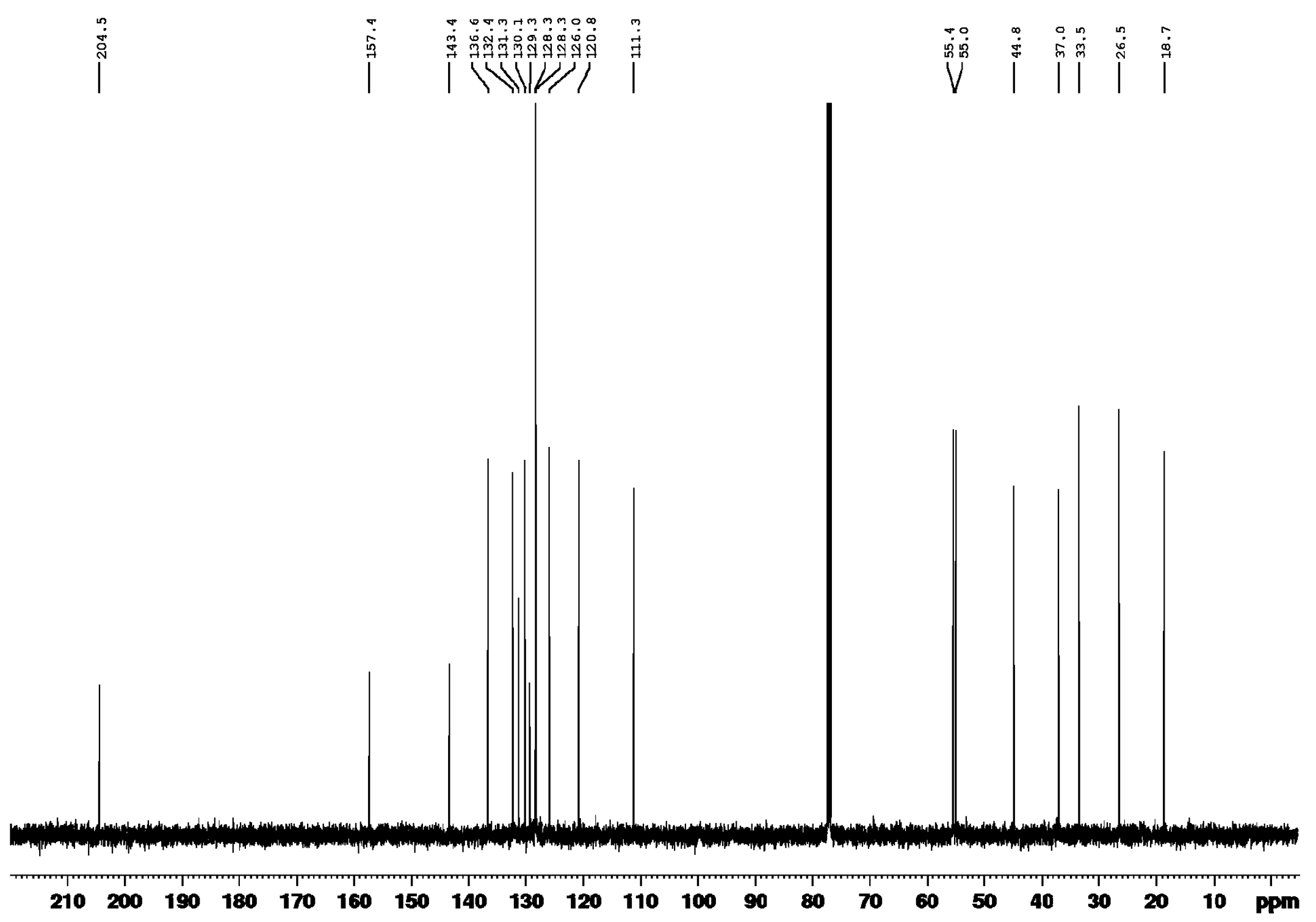


Supporting Information for Organometallic

S94

Figure S64. ${ }^{1} \mathrm{H}$ NMR $\left(500 \mathrm{MHz}, \mathrm{CDCl}_{3}\right)$ of 27.
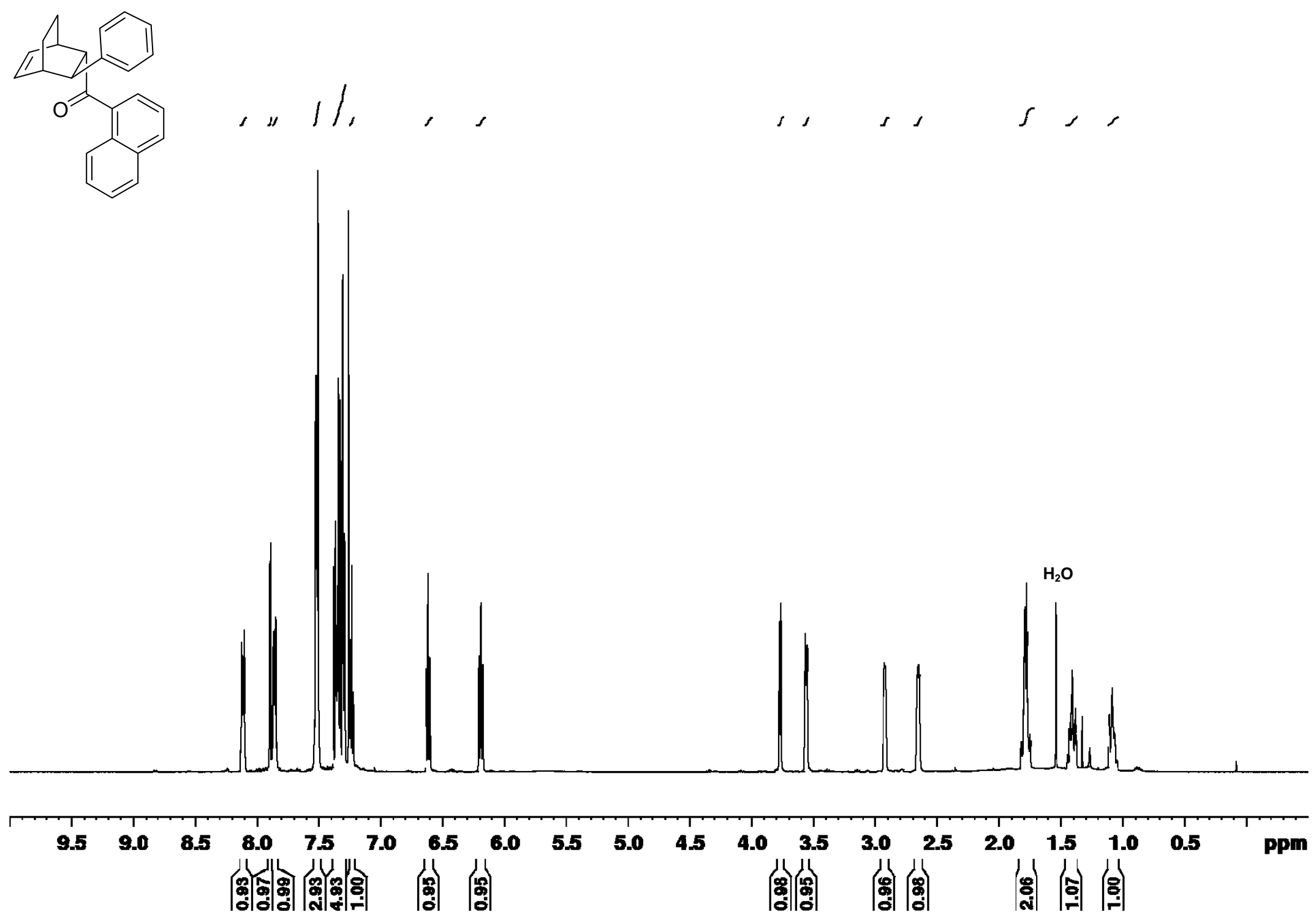
Figure S65. ${ }^{13} \mathrm{C}$ NMR $\left(126 \mathrm{MHz}, \mathrm{CDCl}_{3}\right)$ of 27.

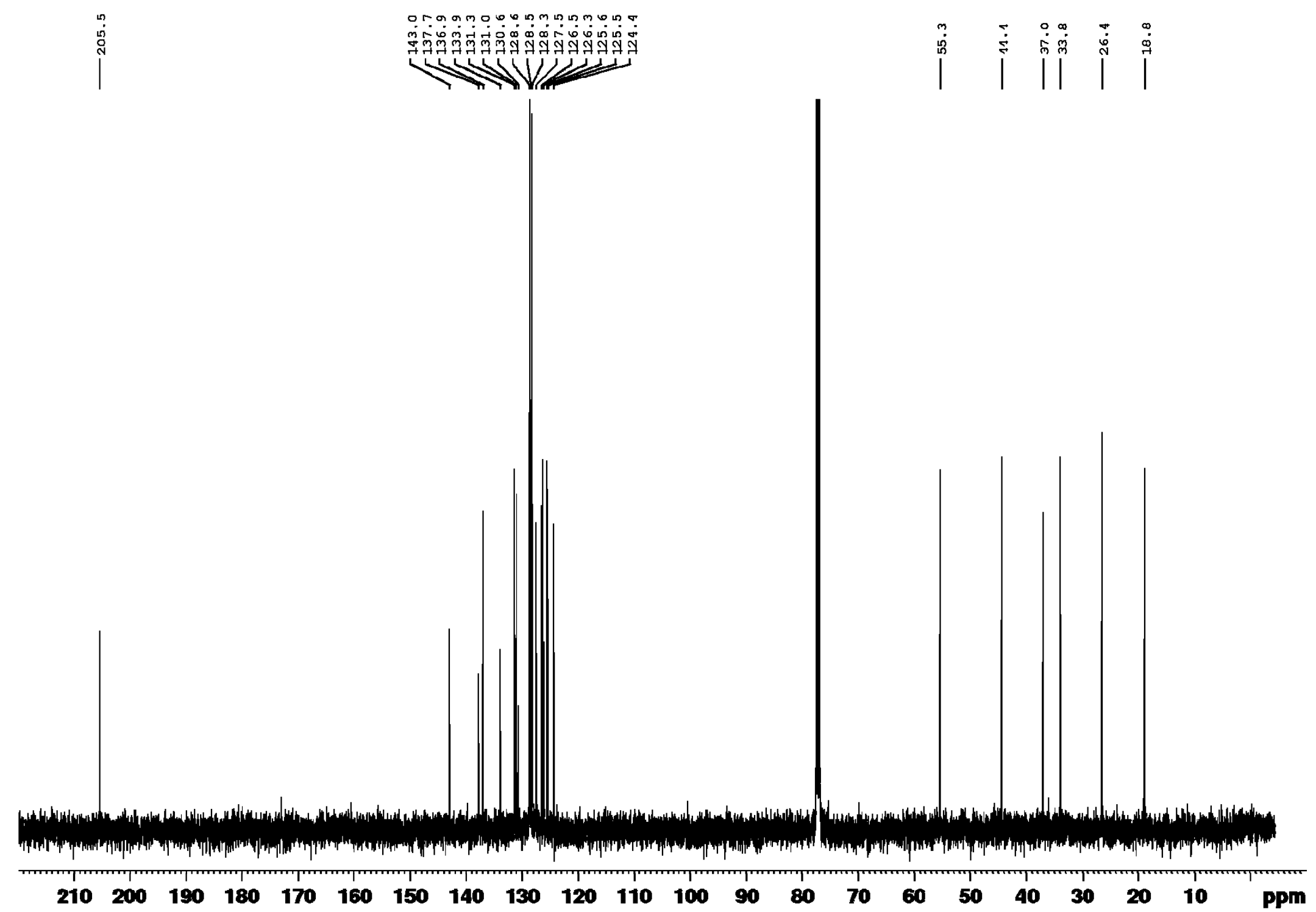


Figure S66. ${ }^{1} \mathrm{H} \mathrm{NMR}\left(500 \mathrm{MHz}, \mathrm{CDCl}_{3}\right)$ of 28.

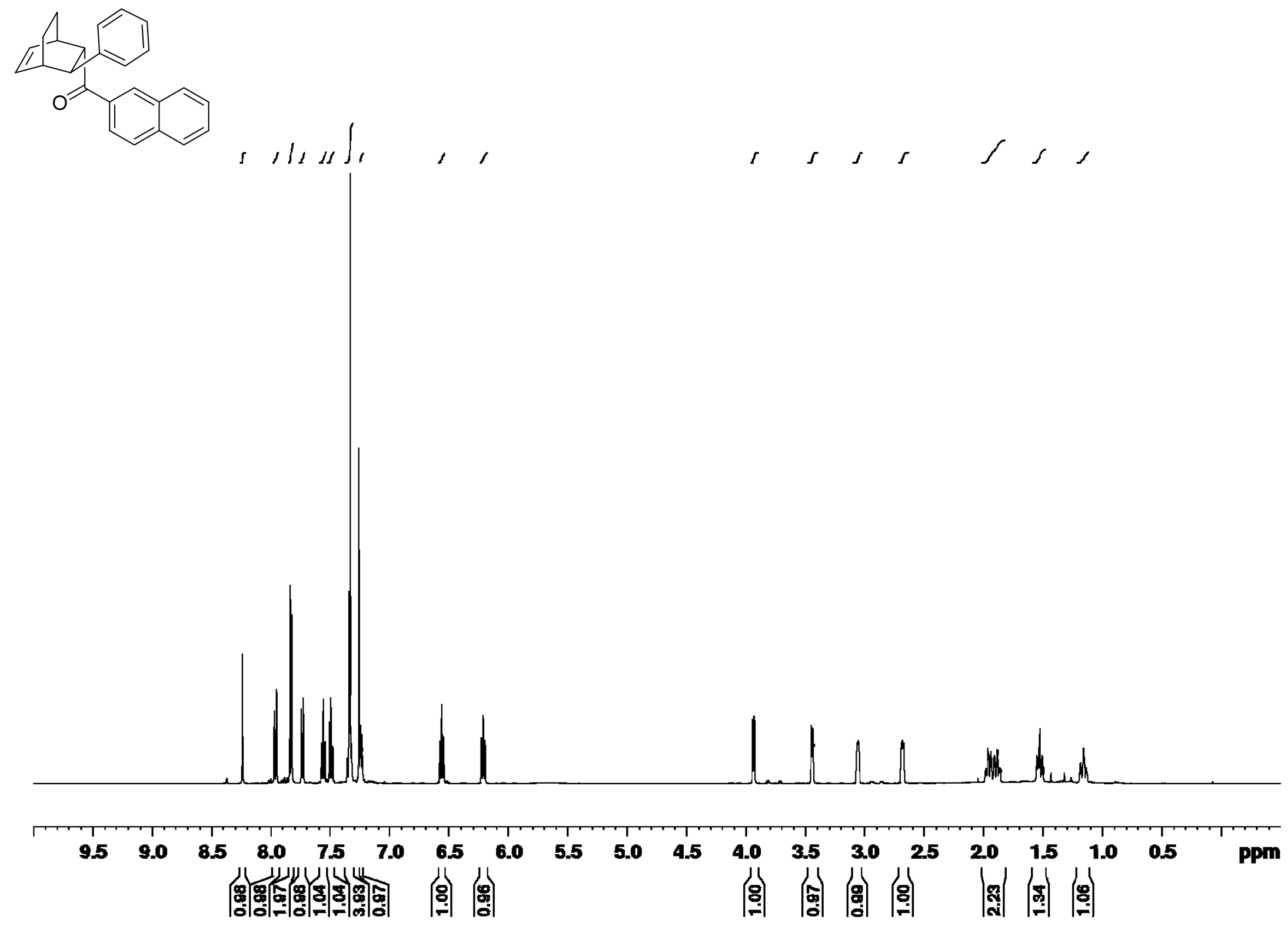


Supporting Information for Organometallic

S97

Figure S67. ${ }^{13} \mathrm{C}$ NMR $\left(126 \mathrm{MHz}, \mathrm{CDCl}_{3}\right)$ of 28.

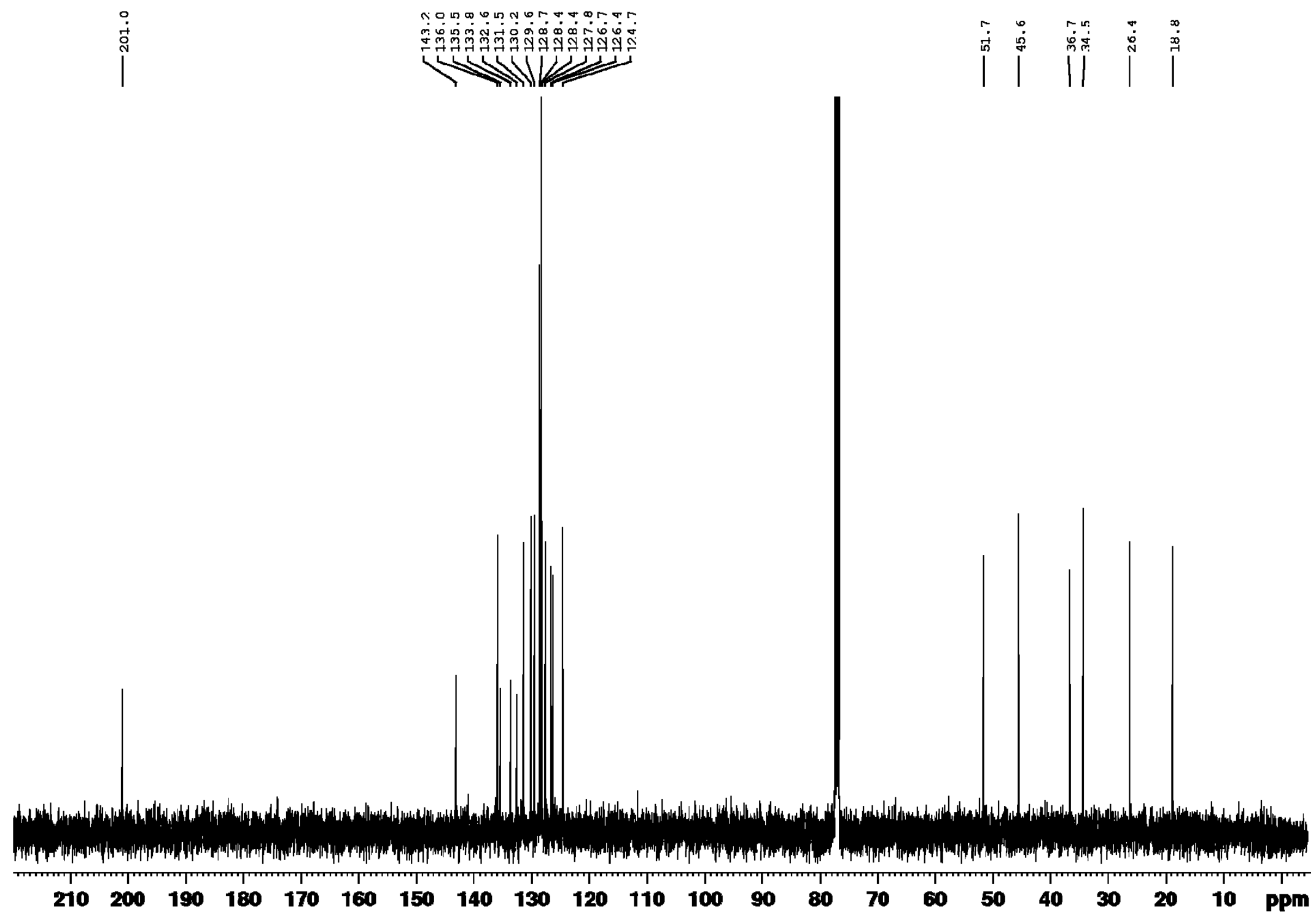


Supporting Information for Organometallic

S98

Figure S68. ${ }^{1} \mathrm{H}$ NMR $\left(500 \mathrm{MHz}, \mathrm{CD}_{2} \mathrm{Cl}_{2}\right)$ of 29.
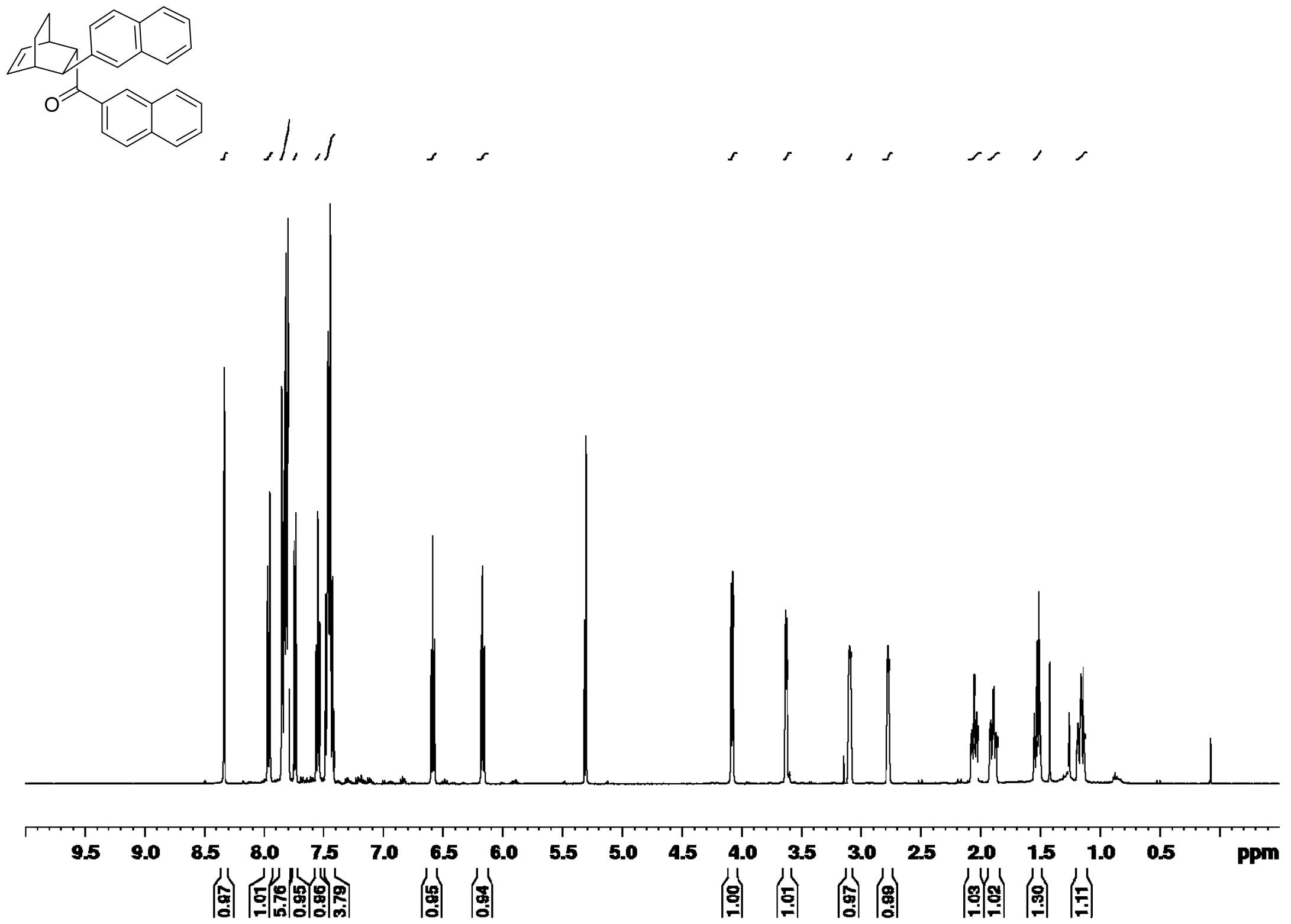
Supporting Information for Organometallics

S99

Figure S69. ${ }^{13} \mathrm{C}$ NMR (126 MHz, $\left.\mathrm{CD}_{2} \mathrm{Cl}_{2}\right)$ of 29.

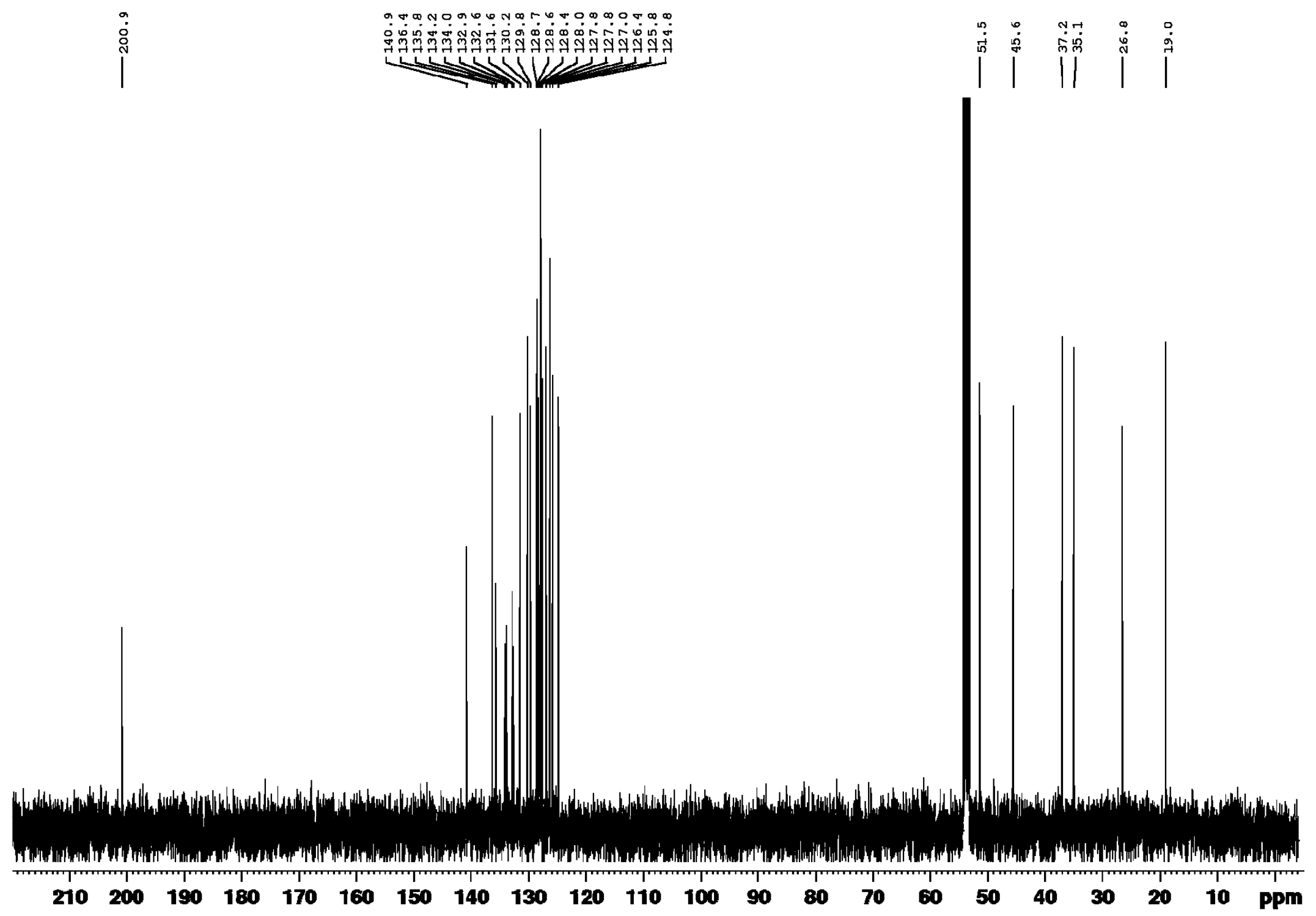


Supporting Information for Organometallic

S100

Figure S70. ${ }^{1} \mathrm{H} \mathrm{NMR}\left(500 \mathrm{MHz}, \mathrm{CDCl}_{3}\right)$ of $\mathbf{3 1}$.
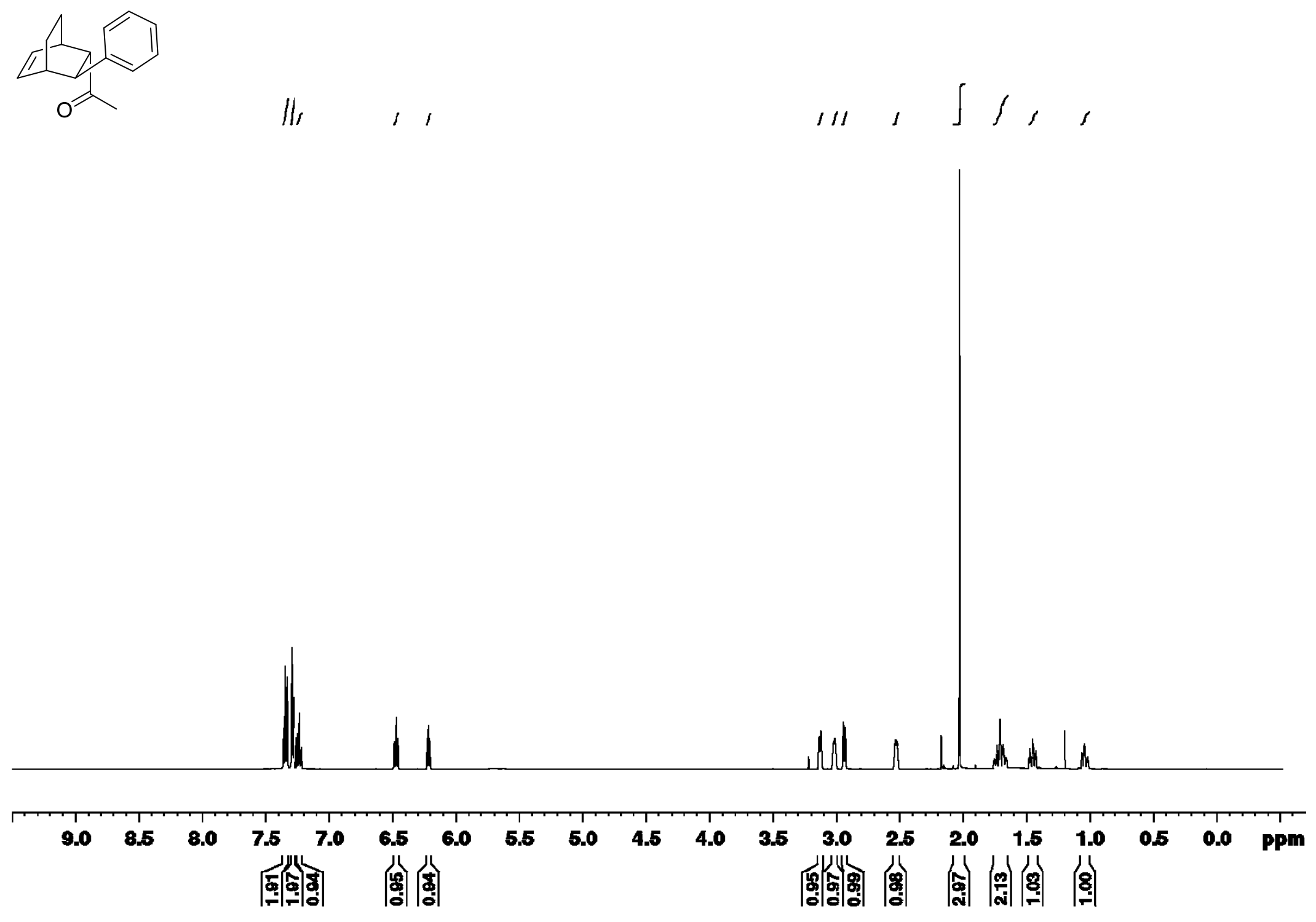
Figure S71. ${ }^{13} \mathrm{C}$ NMR $\left(126 \mathrm{MHz}, \mathrm{CDCl}_{3}\right)$ of $\mathbf{3 1}$.

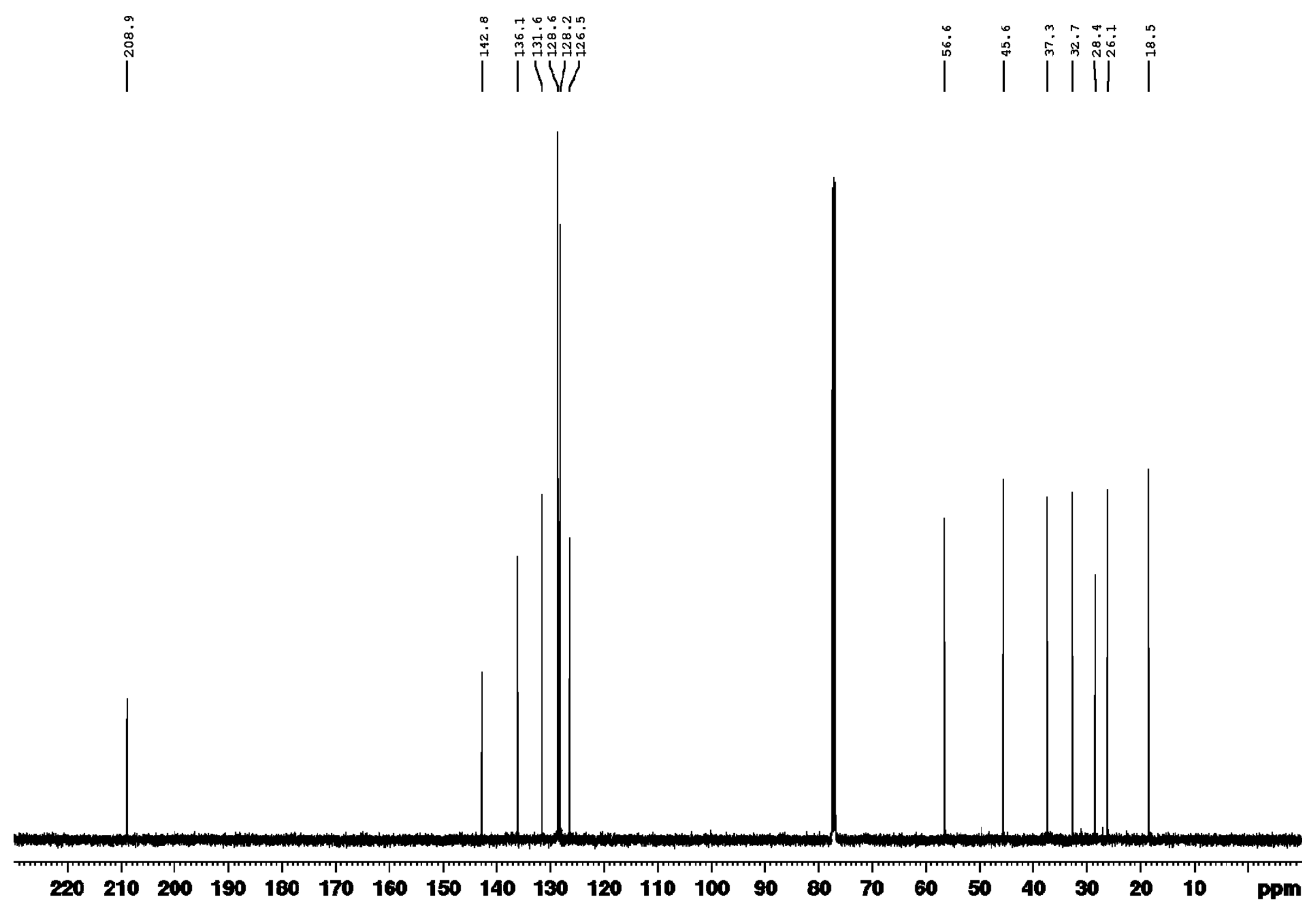




\section{$7 \quad$ References}

[S1] (a) Wang, C.; Erker, G.; Kehr, G.; Wedeking, K.; Fröhlich, R. Organometallics 2005, 24, 4760-4773. (b) Lambert, J. B.; Lin, L.; Keinan, S. Org. Biomol. Chem. 2003, 1, 2559-2565.

[S2] Hoshi, T.; Sasaki, K.; Sato, S.; Ishii, Y.; Suzuki, T.; Hagiwara, H. Org. Lett. 2011, 13, 932935.

[S3] Rohde, V. H. G.; Müller, M. F.; Oestreich, M. Organometallics 2015, 34, 3358-3373. 